Jörn Große-Knetter und Peter Schaaf

\title{
Das Physikalische Praktikum Band II
}

Handbuch 2015/2016 für Studentinnen und Studenten der Physik

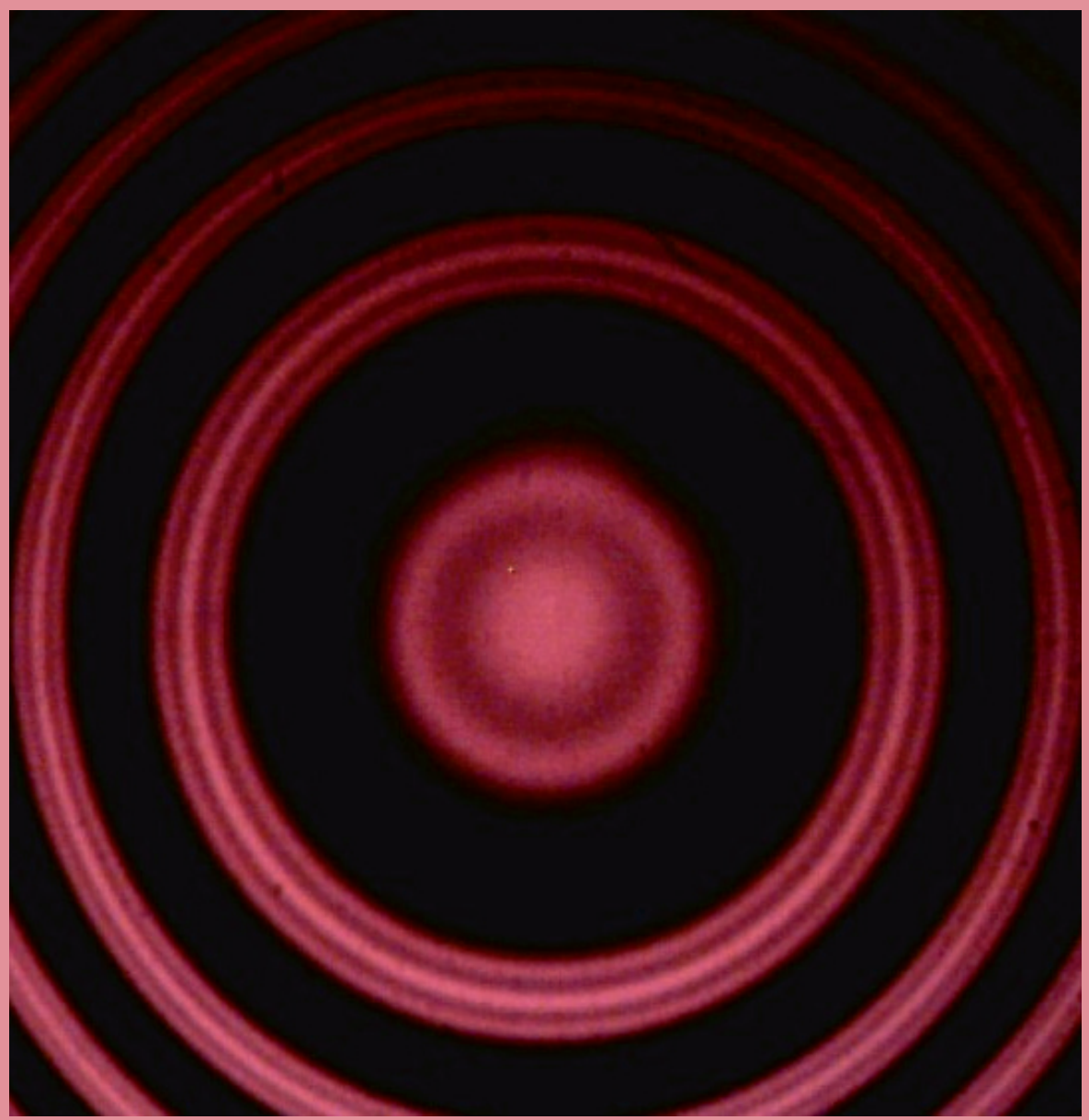

\section{Universitätsdrucke Göttingen}



Jörn Große-Knetter und Peter Schaaf

Das Physikalische Praktikum - Band II

Dieses Werk ist lizenziert unter einer

Creative Commons

Namensnennung - Weitergabe unter gleichen Bedingungen

4.0 International Lizenz.

(c) (1) (2) 
erschienen in der Reihe der Universitätsdrucke im Universitätsverlag Göttingen 2015 
Jörn Große-Knetter

und Peter Schaaf

Das Physikalische

Praktikum

Band II

Handbuch 2015/2016

für Studentinnen und Studenten der Physik

Mit 35 Abbildungen und 14 Tabellen

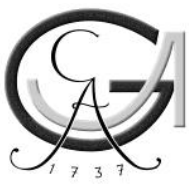

Universitätsverlag Göttingen 2015 


\section{Bibliographische Information der Deutschen Nationalbibliothek}

Die Deutsche Nationalbibliothek verzeichnet diese Publikation in der Deutschen Nationalbibliographie; detaillierte bibliographische Daten sind im Internet über $<$ http://dnb.dnb.de $>$ abrufbar.

Adresse der Autoren

Zweites Physikalisches Institut

Universität Göttingen

Friedrich-Hund-Platz 1

D-37077 Göttingen

Tel.: 0551 39-7632

E-Mail: jgrosse1@uni-goettingen.de

URL: http://www.praktikum.physik.uni-goettingen.de/

Dieses Buch ist auch als freie Onlineversion über die Homepage des Verlags sowie über den Göttinger Universitätskatalog (GUK) bei der Niedersächsischen Staats- und Universitätsbibliothek Göttingen (http://www.sub.uni-goettingen.de) erreichbar. Es gelten die Lizenzbestimmungen der Onlineversion.

Satz und Layout: LATEX von Jörn Große-Knetter

Titelabbildung: Interferenzmuster zum Versuch »Zeeman-Effekt«

(C) 2015 Universitätsverlag Göttingen

http://univerlag.uni-goettingen.de

ISBN: 978-3-86395-249-5 


\section{Inhaltsverzeichnis}

Vorwor ............................. . . . . . . .

\begin{tabular}{lll}
\hline I $\quad$ Vorbemerkungen & 1
\end{tabular}

A Literatur für das Praktikum . . . . . . . . . . . . . . . . 2

B Organisatorische Regeln für das Praktikum . . . . . . . . . . . 5

C Sicherheit im Praktikum . . . . . . . . . . . . . . . . . 12

$\begin{array}{lr}\text { II Versuche } & 15\end{array}$

12 Ultraschallwellen . . . . . . . . . . . . . . . . . . . . . . . 16

13 Mikrowellen . . . . . . . . . . . . . . . . . . . . . . . . . . . 20

14 Das Mikroskop . . . . . . . . . . . . . . . . . . . . . . . . . . . . . . . . . . 24

15 Messung der Lichtgeschwindigkeit . . . . . . . . . . . . . . . . . . . . . . . . 30

16 Das Prismen- und Gitterspektrometer . . . . . . . . . . . . . . . . . . . . . 34

17 Fresnelsche Formeln und Polarisation $\ldots \ldots \ldots \ldots$. . . . . . . . . . . . . . . . . . .

18 Beugung und Interferenz von Laserlicht . . . . . . . . . . . . . . . . . . . 48

19 Die spezifische Elektronenladung . . . . . . . . . . . . . . . . . . . . . . . 64

20 Der Franck-Hertz-Versuch $\ldots \ldots \ldots$. . . . . . . . . . . . . . . . 68

21 Balmerserie von Wasserstoff . . . . . . . . . . . . . . . . . . . . 72

22 Röntgenstrahlung . . . . . . . . . . . . . . . . . . . . . . . . . . 75

23 Zeeman-Effekt . . . . . . . . . . . . . . . . . . . . . . . . . . . . 84

24 Elektronenspinresonanz . . . . . . . . . . . . . . . . . . . . . . . . . . 91

25 Radioaktivität . . . . . . . . . . . . . . . . . . . . . . . 95

\begin{tabular}{lr}
\hline Anhang & 105
\end{tabular}

Literaturverzeichnis . . . . . . . . . . . . . . . . . . . . . . . . . 106

Abbildungsverzeichnis . . . . . . . . . . . . . . . . . . . . 110

Tabellenverzeichnis . . . . . . . . . . . . . . . . . . . . . . . . . 112

Raumverzeichnis des Praktikums . . . . . . . . . . . . . . . . . . . . . . . . 113

Stichwortverzeichnis . . . . . . . . . . . . . . . . . . . . . . 115 



\section{Vorwort WiSe 2015/16}

Herzlich Willkommen zum Praktikum zu Experimentalphysik an der Universität Göttingen. Dieses »Handbuch« (oder auch Praktikumsanleitung) wird Sie die nächsten zwei Semester begleiten. Das Grundpraktikum für das Fach Physik wird begleitend zu den Vorlesungen Experimentalphysik I...IV durchgeführt und beinhaltet die Einführungsveranstaltung »Grundlagen des Experimentierens « (GdE) sowie 25 Versuche. Dieser Band umfasst die 14 Versuche zu Experimentalphysik III und IV, welche im Regelfall im 3. und 4. Semester absolviert werden.

Das Praktikum zu den Modulen B.phy.1101 bis 1104 wird benötigt für den Bachelor in Physik. Dieses Praktikum wird mit 3 SWS pro Abschnitt (Semester) angerechnet.

Das Göttinger Physikpraktikum für angehende Physikerinnen und Physiker führt anhand ausgewählter, vorgefertigter Versuche in einen weiten Bereich physikalischer Grundlagen, in den Umgang mit Apparaturen und Messgeräten und in die Technik des physikalischen Experimentierens ein und stellt damit einen wesentlichen Teil der traditionellen Grundausbildung in Physik dar. Ziel ist hierbei auch eine Vertiefung des bisher in den Vorlesungen erlernten Stoffes durch eigenes Umsetzen und das Erfahren von Physik (learning by doing). Sie erlernen den Umgang mit verschiedensten Geräten und erfahren durch eigenes Tun, wie eine physikalische Aufgabenstellung experimentell und methodisch angegangen wird (hands on physics). Problem - Analyse - Bearbeitung - Lösung - Dokumentation, dies ist die Sequenz, die Sie in Ihrem ganzen »PhysikLeben « begleiten wird. Hierbei spielt auch Gruppen- oder Teamarbeit eine wichtige Rolle. Nutzen Sie die Gelegenheit im Praktikum auch dies zu üben, und bringen Sie sich aktiv ein. Es wird sich auszahlen.

Dieses Handbuch beschreibt derzeit 14 Versuche, die verpflichtend zu den Veranstaltungen Experimentalphysik III und IV durchgeführt werden müssen. Die vorhergehenden 11 Versuche zu Experimentalphysik I und II werden im ersten Band zusammen mit einführenden Konzepten beschrieben, die auch für diesen Abschnitt weiterhin berücksichtigt werden sollen.

Wir legen hiermit die erste Ausgabe des zweiten Bands vor, welche insbesondere die Änderungen im Studienverlauf und die Angliederung des Praktikums an die ExperimentalphysikVorlesungen mit entsprechenden Anpassungen und Erweiterungen der Versuche widerspiegelt. Es ist nur natürlich, dass sich damit neue Fehler und Unzulänglichkeiten in dieses Handbuch eingeschlichen haben. Wir wären dankbar, wenn Sie uns Fehler und auch Verbesserungsvorschläge sofort mitteilen würden (E-Mail: jgrosse1@uni-goettingen.de). Wir werden diese dann schnellstmöglich beheben und auf den Web-Seiten des Praktikums eine verbesserte Version der jeweiligen Anleitung zur Verfügung stellen. Noch sind nicht alle Versuche und ihre Anleitungen auf dem Stand, den Sie und wir gerne hätten. Dies werden Sie sicherlich feststellen. Bedenken Sie, dass diese Anleitung und auch die Überarbeitung und Erneuerung der Versuche sehr viel Arbeit erfordert, und wir bei der derzeitigen Personal- und Betreuungssituation nicht alles umsetzen können, was Sie sich und wir uns wünschen.

Auch nach der Modernisierung im Zuge der Umstellung auf das Praktikum zu Experimentalphysik soll dies weiterhin kontinuierlich durch Neuanschaffungen, Versuchsmodifikationen und Entwicklung neuer Versuche geschehen. Daneben gibt es Apparaturen, die etwas »altmodi- 
scher « aussehen, aber doch noch ganz ihrer (didaktischen) Aufgabe gerecht werden. Es kostet große Mühe, alle diese Apparaturen in einem einwandfreien Zustand zu erhalten. Sollten Sie dennoch Fehler feststellen, so geben Sie uns bitte sofort Bescheid. Nur dann können wir für Abhilfe sorgen.

Auch nach dem Druck dieser »Praktikumsanleitung « werden Versuche weiterentwickelt und verbessert, so dass es zu Abweichungen des aktuellen Versuches von dieser Anleitung kommen kann. Bedenken Sie bitte, dass zwischen Drucklegung und Ihrer Durchführung des Versuches schon eine lange Zeitspanne vergangen sein kann (im Extremfall über ein Jahr). Wir bemühen uns, Ihnen solche Änderungen und Verbesserungen rechtzeitig mitzuteilen, hoffen aber auch, dass Sie diese Verbesserungen honorieren werden. Wir werden versuchen, auf den Webseiten immer aktuelle Versuchsanleitungen zur Verfügung zu stellen. Es lohnt sich also bestimmt, von Zeit zu Zeit auf den Web-Seiten des Praktikums http://www.praktikum.physik.uni-goettingen.de nachzusehen, da wir uns bemühen werden, dort immer die aktuellsten Informationen zu publizieren.

Bitte bedenken Sie auch immer, dass Ihre Betreuerinnen und Betreuer für Ihr Praktikum, also für Ihren Lernerfolg, viel Arbeit und Zeit investieren. Dies geschieht neben eigenem Studium oder eigener Promotion und resultiert in einer Belastung, die weit über das hinausgeht, was als Lehrverpflichtung von Betreuer(innen) im Durchschnitt an der Fakultät erbracht wird. Leider stehen uns nicht so viele Betreuer(innen) zur Verfügung, wie wir dies aus praktischen und didaktischen Erwägungen für sinnvoll erachten. Erleichtern Sie deshalb bitte Ihren Betreuerinnen und Betreuern diese Belastung durch Ihre engagierte, aktive, gut vorbereitete und möglichst eigenständige Mitarbeit im Praktikum und »zahlen« Sie deren Engagement mit Ihrem persönlichen guten Lernerfolg zurück. Nur Ihr aktives und eigenständiges Arbeiten erzielt auch eine hohe Nachhaltigkeit des Erlernten und schafft so das solide Wissensfundament, auf dem Sie Ihre Zukunft aufbauen können.

Zusammenfassend wünschen Ihnen alle Betreuerinnen und Betreuer des Praktikums viel Spaß im und einen guten Lernerfolg durch das Praktikum. Wir alle, insbesondere Ihre Betreuerinnen und Betreuer, bemühen uns, damit dies - Ihre Mithilfe angenommen - auch erreicht werden kann. 


\section{Teil I}

\section{Vorbemerkungen}




\section{A Literatur für das Praktikum}

Als begleitende Literatur für das Physikalische Praktikum sind prinzipiell alle Physikbücher geeignet. Insbesondere sind die folgenden Bücher zu nennen. Die Aufzählung erhebt weder einen Anspruch auf Vollständigkeit, noch stellt sie eine Wertung dar. Welches Buch für Sie persönlich das Beste ist, können nur Sie selbst entscheiden. Schauen Sie sich die Bücher an, vergleichen Sie dabei beispielsweise direkt die unterschiedlichen Darstellungen eines bestimmten engen Gebietes. Wählen Sie dann dasjenige Buch aus, welches Ihnen am besten liegt. Neben dieser Aufzählung finden sich diese und weitere Bücher auch im Literaturverzeichnis wieder. Die Abkürzungen der Bücher werden zum Teil auch bei den Versuchen zur Angabe vertiefender Literatur benutzt.

Wählen Sie anhand der Sachverzeichnisse und der Stichworte in den Anleitungen die geeignete Literatur zum jeweiligen Versuch aus. Zu einigen Versuchen wird spezielle Literatur angegeben. Die meisten Bücher sind in der Bereichsbibliothek Physik BBP ausleih- oder einsehbar. Wir sind bemüht, die wichtigsten physikalischen Grundlagen in diese Anleitung aufzunehmen. Dies ist aber erst für einige Versuche gelungen. Bitte eignen Sie sich selbständig die zugehörige Physik durch Nachlesen in mehreren Büchern (zur Not auch in Vorgängerprotokollen, aber auf Richtigkeit achten!) tiefgehender an. Die unterschiedlichen Darstellungsweisen fördern das Verständnis.

\section{A.1 Spezielle Praktikumsbücher}

Tabelle A.1 enthält eine Aufzählung von Büchern, die speziell für Physikalische Praktika gedacht sind (Praktikumsbücher) und somit auch Methodisches und Handlungshinweise enthalten.

\section{A.2 Allgemeine Physikbücher}

Folgende, in Tabelle A.2 aufgeführte, allgemeine Physikbücher sind für das Praktikum und das Studium insgesamt nützlich.

Tabelle A.1: Dedizierte Praktikumsbücher

\begin{tabular}{|c|c|}
\hline Kürzel & Autor, Titel, Verlag, Jahr, Referenz \\
\hline NPP & $\begin{array}{l}\text { Eichler, Kronfeld, Sahm, Das Neue Physikalische Grundpraktikum, Springer, } 2006 \\
{[17]}\end{array}$ \\
\hline Schenk & ScHENK, Physikalisches Praktikum, Springer Spektrum, 2014 [59] \\
\hline Wal & WALCHER, Praktikum der Physik, Teubner, 2004 [66] \\
\hline Wes & Westphal, Praktikum der Physik, Springer, 1984 (vergriffen) 69] \\
\hline BeJo & BECKER, JodL, Physikalisches Praktikum, VDI-Verlag, 1983 [2] \\
\hline CIP & Diemer, Basel, Jod, Computer im Praktikum Springer, 1999 [13] \\
\hline Paus & Paus, Physik in Experimenten und Beispielen, Hanser [48] \\
\hline
\end{tabular}


Tabelle A.2: Allgemeine Physikbücher, die für das Praktikum nützlich sind.

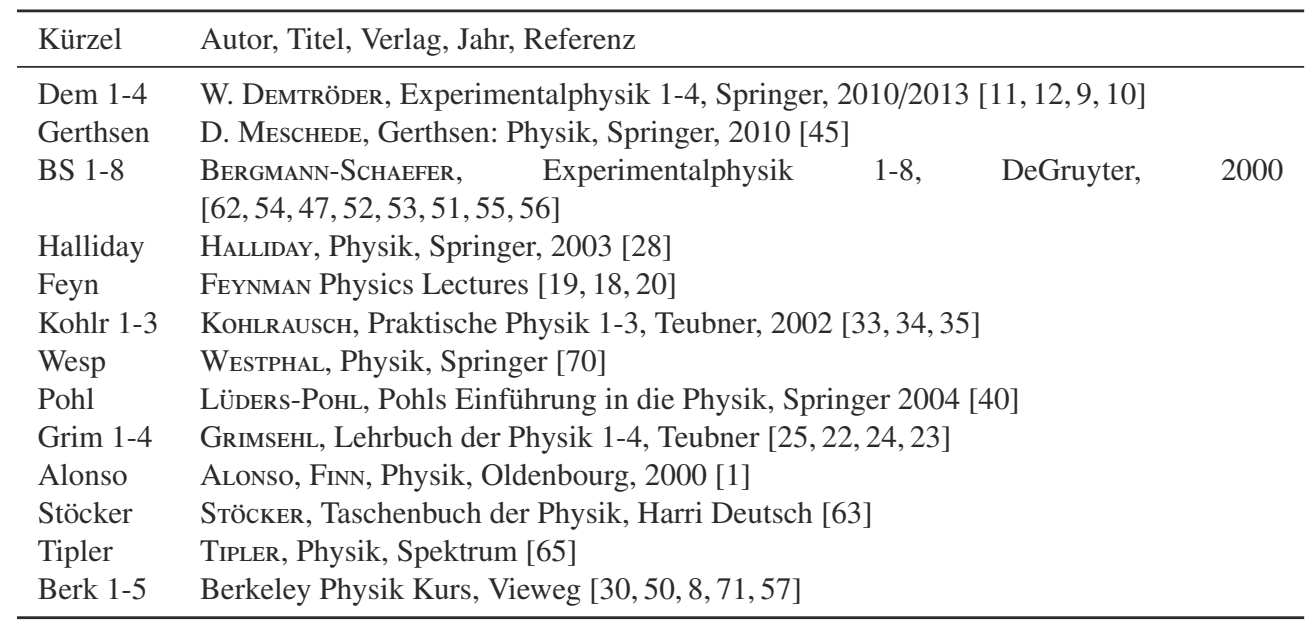

\section{A.3 Handbücher und Nachschlagewerke}

Nützliche Hinweise zur Auswertung und Fehlerrechnung, sowie eine Vielzahl von Werten und Materialdaten, findet man in den in Tabelle A.3 aufgeführten Nachschlagewerken.

Tabelle A.3: Handbücher und Nachschlagewerke für das Praktikum

\begin{tabular}{|c|c|}
\hline Kürzel & Autor, Titel, Verlag, Jahr, Referenz \\
\hline Bron & Bronstein-Semendajev, Taschenbuch der Mathematik, H. Deutsch [5] \\
\hline TBMathe & $\begin{array}{l}\text { STöскER, Taschenbuch mathematischer Formeln u. moderner Verfahren, H. Deutsch } \\
\text { [64] }\end{array}$ \\
\hline TBPhys & Stöcker, Taschenbuch der Physik, H. Deutsch 63] \\
\hline TBRegel & Lutz, Wendt, Taschenbuch der Regelungstechnik, H. Deutsch [41] \\
\hline TBStat & RinNe, Taschenbuch der Statistik, H. Deutsch [58] \\
\hline TBElektro & Kories, Schmidt-WaLter, Taschenbuch der Elektrotechnik, H. Deutsch [14] \\
\hline TBChem & Schröter, LaUtenschläGER, Bibrack, Taschenbuch d. Chemie, H. Deutsch [60] \\
\hline Kneu & KNEUBÜHL, Repetitorium der Physik, Teubner [31] \\
\hline Lichten & Lichten, Scriptum Fehlerrechnung, Springer [38] \\
\hline Beving & $\begin{array}{l}\text { Bevington, Robinson, Data reduction and error analysis for the physical sciences, } \\
\text { McGraw-Hill, } 1992 \text { [4] }\end{array}$ \\
\hline Tab & Berber, KaChER, LANGER, Physik in Formeln und Tabellen, Teubner [3] \\
\hline Messunsicher & $\begin{array}{l}\text { WeISE, WöGER, Messunsicherheit und Messdatenauswertung, Wiley-VCH, Weinheim, } \\
1999 \text { 68 }\end{array}$ \\
\hline UmgUnsich & DrosG, Der Umgang mit Unsicherheiten, facultas, 2006 [16] \\
\hline Kunze & Kunze, Physikalische Messmethoden, Teubner, 1986 [37] \\
\hline LandBörn & LANDOLT-BÖRNSTEIN WWW. springeronline. de [42] \\
\hline NIST & NIST WWW.nist.gov \\
\hline
\end{tabular}




\section{A.4 Fundamentalkonstanten}

Viele physikalische Fundamentalkonstanten werden im Praktikum für Berechnungen benötigt oder werden dort gemessen. Tabelle A.4 gibt eine Auswahl aus der von der IUPAP (International Union of Pure and Applied Physics) festgelegten Zusammenstellung CODATA [6] wieder.

Tabelle A.4: Wichtige physikalische Fundamentalkonstanten [6]. $\Delta x / x$ ist die relative Unsicherheit (ppm parts per million, $\times 10^{-6}$ ).

\begin{tabular}{|c|c|c|c|}
\hline Konstante & Symbol & Wert & $\Delta x / x[\mathrm{ppm}]$ \\
\hline Vakuumlichtgeschwindigkeit & $c_{0}$ & $299792458 \mathrm{~m} \mathrm{~s}^{-1}$ & exakt \\
\hline Permeabilität des Vakuums & $\mu_{0}$ & $4 \pi \cdot 10^{-7} \mathrm{NA}^{-2}$ & exakt \\
\hline Permittivität des Vakuums & $\epsilon_{0}$ & $8,854187817 \cdot 10^{-12} \mathrm{Fm}^{-1}$ & exakt \\
\hline Gravitationskonstante & $G, \gamma$ & $6,67259 \cdot 10^{-11} \mathrm{~m}^{3} \mathrm{~kg}^{-1} \mathrm{~s}^{-2}$ & 128 \\
\hline \multirow[t]{2}{*}{ Planck Konstante } & $h$ & $6,6260755 \cdot 10^{-34} \mathrm{~J} \mathrm{~s}$ & 0,60 \\
\hline & $h$ & $4,1356692 \cdot 10^{-15} \mathrm{eV} \mathrm{s}$ & 0,30 \\
\hline Elementarladung & $e$ & $1,60217733 \cdot 10^{-19} \mathrm{C}$ & 0,30 \\
\hline Hall-Widerstand & $R_{\mathrm{H}}$ & $25812,8056 \Omega$ & 0,045 \\
\hline Bohr Magneton & $\mu_{\mathrm{B}}=e \hbar / 2 m_{e}$ & $5,78838263 \cdot 10^{-5} \mathrm{eV} / \mathrm{T}$ & 0,089 \\
\hline \multirow{2}{*}{ Feinstrukturkonstante } & $\alpha=\mu_{0} c e^{2} / 2 h$ & 0,00729735308 & 0,045 \\
\hline & $\alpha^{-1}$ & 137,0359895 & 0,045 \\
\hline \multirow[t]{3}{*}{ Rydberg Konstante } & $R_{\infty}$ & $10973731,534 \mathrm{~m}^{-1}$ & 0,0012 \\
\hline & $c R_{\infty}$ & $3,2898419499 \cdot 10^{15} \mathrm{~Hz}$ & 0,0012 \\
\hline & $h c R_{\infty}$ & $13,6056981 \mathrm{eV}$ & 0,30 \\
\hline Bohr Radius & $a_{0}=\alpha / 4 \pi R_{\infty}$ & $0,529177249 \cdot 10^{-10} \mathrm{~m}$ & 0,045 \\
\hline Elektronenmasse & $m_{e}$ & $9,1093897 \cdot 10^{-31} \mathrm{~kg}$ & 0,59 \\
\hline Avogadro Konstante & $N_{\mathrm{A}}$ & $6,0221367 \cdot 10^{23} \mathrm{~mol}^{-1}$ & 0,59 \\
\hline \multirow[t]{2}{*}{ Atomare Masseneinheit } & $m_{u}$ & $1,6605402 \cdot 10^{-27} \mathrm{~kg}$ & 0,59 \\
\hline & $m_{u}$ & $931,49432 \mathrm{MeV}$ & 0,30 \\
\hline Faraday Konstante & $F$ & $96485,309 \mathrm{C} \mathrm{mol}^{-1}$ & 0,30 \\
\hline Molare Gaskonstante & $R$ & $8,314510 \mathrm{~J} \mathrm{~mol}^{-1} \mathrm{~K}^{-1}$ & 8,4 \\
\hline \multirow[t]{2}{*}{ Boltzmann Konstante } & $k_{\mathrm{B}}$ & $1,380658 \cdot 10^{-23} \mathrm{~J} \mathrm{~K}^{-1}$ & 8,5 \\
\hline & $k_{\mathrm{B}}$ & $8,617385 \cdot 10^{-5} \mathrm{eV} \mathrm{K}^{-1}$ & 8,4 \\
\hline Molvolumen Ideales Gas (Normalbed.) & $V_{\mathrm{m}}$ & $22414,10 \mathrm{~cm}^{3} \mathrm{~mol}^{-1}$ & 8,4 \\
\hline Loschmidt Konstante & $n_{0}=N_{A} / V_{m}$ & $2,686763 \cdot 10^{25} \mathrm{~m}^{-3}$ & 8,5 \\
\hline Stefan-Boltzmann Konstante & $\sigma$ & $5,67051 \cdot 10^{-8} \mathrm{~W} \mathrm{~m}^{-2} \mathrm{~K}^{-4}$ & 34 \\
\hline Wien Konstante & $b=\lambda_{\max } T$ & $0,002897756 \mathrm{~m} \mathrm{~K}$ & 8,4 \\
\hline
\end{tabular}




\section{B Organisatorische Regeln für das Praktikum}

Das Praktikum zu Experimentalphysik (Praktikumsteil der Module B.Phy.1101 bis 1104) besteht in den dritten und vierten Teilen, welche im dritten bzw. vierten Semester durchgeführt werden (B.Phy.1103.2 und B.Phy.1104.2), aus je 7 Versuchen (jeweils 3 SWS in beiden Semestern). Alle Versuche werden während der Vorlesungszeit durchgeführt, um eine zeitlich enge Bindung an die Vorlesung zu erzielen.

Es gelten folgende organisatorische Regeln für das Praktikum, die einen reibungslosen und effektiven Ablauf des Praktikums ermöglichen sollen. Diese sind immer zu beachten.

\section{B.1 Anmeldung}

Eine persönliche Anmeldung ist für die Versuchsdurchführung erforderlich. Zu diesem Zweck werden in der zugehörigen StudIP-Veranstaltung Versuchsgruppen zu Semesterbeginn angelegt. Eine Teilnahme an den Versuchen ist nur bei einem entsprechenden Gruppeneintrag möglich.

\section{B.2 Versuchsvorbereitung}

Jede Praktikantin und jeder Praktikant muss sich genügend auf den durchzuführenden Versuch vorbereiten. Die Durcharbeitung der Anleitung zum Praktikum und das Literaturstudium sind obligatorisch. Auch ist es äußerst hilfreich, sich die Versuchsapparatur vor dem Praktikumstag anzuschauen (z.B. am Versuchstag davor) und sich schon vorher zu überlegen, was gemessen werden muss (Tabellen erstellen). Es empfiehlt sich, den Theorieteil für das Protokoll schon vor dem Versuch zu schreiben, da dies auch erfahrungsgemäß die benötigte Zeit zur Protokollerstellung drastisch reduziert.

Lesen Sie die jeweilige Versuchsanleitung bitte bis zum Ende. Insbesondere der letzte Abschnitt »Bemerkungen« enthält meist wichtige Hinweise für die Versuchsdurchführung.

Wer unvorbereitet zu einem Versuch kommt, riskiert, dass er/sie den Versuch an diesem Tag nicht durchführen darf und einen Nachholtermin in Anspruch nehmen muss. Eine ungenügende Vorbereitung wird von der Betreuerin/dem Betreuer sehr schnell erkannt. Es werden auch Stichproben während des Praktikums durch die Praktikumsleitung stattfinden.

Die Betreuerinnen und Betreuer sind gehalten, vor jedem Versuch nochmals die Sicherheitsaspekte zum Versuch zu erläutern und deren Verständnis zu überprüfen. 


\section{B.3 Theorievortrag}

Die Vorbesprechung des Versuchs beginnt gewöhnlich um 14:15 Uhit. Erheblich verspätetes Erscheinen führt zum Ausschluss an der Durchführung des Praktikums am jeweiligen Tag. In jedem Abschnitt an Versuchen (Modulteil) muss jede/r Praktikant/in mindestens einmal einen kleinen (Theorie-)vortrag (ca. 15 min.) über den Versuch gehalten haben, wobei die Vorträge zu zweit, sinnvollerweise mit dem/der Messpartner(in), gehalten werden können 2 Der Vortrag sollte einen kurzen Überblick über die Grundlagen und die Durchführung des Versuchs geben. Es ist zu empfehlen, sich vorher mit dem/der Betreuer(in) abzusprechen, wie ausführlich einzelne Themen angesprochen und erklärt werden sollen. Grundsätzlich sollte der Vortrag nicht länger als 30 Minuten dauern, aber dies ist auch vom Umfang des jeweiligen Versuchs abhängig. Wichtig: Auch wenn nur zwei Praktikanten einen Vortrag halten - die dem Versuch zugrunde liegenden physikalischen Phänomene müssen jedem bekannt sein und von jedem erklärt werden können. Insbesondere muss jede/r Praktikant/in den Gang der Versuchsdurchführung erläutern können. Ist dies nicht der Fall, wird die/der Praktikant/in an der Durchführung des Praktikums an dem jeweiligen Tag ausgeschlossen! Zu jedem Versuch werden in einem entsprechend benannten Abschnitt Fragen zum Versuch aufgelistet, die jede(r) Teilnehmer(in) am Versuch beantworten können muss. Die Betreuerinnen und Betreuer sind angehalten in der Theorie allen Gruppenmitgliedern mindestens eine Frage zu stellen. Mitunter sind weitergehende Fragen (Abschnitt »Weiterführendes«) aufgeführt, die optional zu beantworten sind.

\section{B.4 Durchführung}

Versuche werden grundsätzlich in Gruppen bestehend aus 2 Personen durchgeführt. Insbesondere bei Nachholterminen muss der Praktikant sicherstellen, einen Mitarbeiter zu haben. Es darf niemals alleine im Praktikum gearbeitet werden. Vor dem Beginn der Messungen mache man sich mit den Apparaturen vertraut, d.h. wie sind welche Messgeräte anzuschließen, wie funktionieren sie, wie werden sie abgelesen, welche Fehler haben sie, bei welchen Apparaturen ist besondere Vorsicht geboten, usw. Insbesondere bei elektrischen Stromkreisen ist darauf zu achten, dass Strom und Spannungen sehr gefährlich sein können! Messgeräte sind vor dem Gebrauch - sofern möglich - auf Funktionsfähigkeit zu testen und auf den richtigen Messbereich einzustellen. Manchmal ist es hilfreich, Schalter und Messgeräte durch Zettel oder ähnliches (z.B. »Post-it«) zu beschriften, um Irrtümer zu vermeiden. Bei elektrischen Schaltungen ist nach dem Aufbau zunächst der Assistent zu benachrichtigen, erst mit dessen Zustimmung wird die Stromversorgung eingeschaltet! Messkurven (z.B. Franck-Hertz) sind während der Versuchsdurchführung grafisch darzustellen. Jeder ist selbst dafür verantwortlich, dass alle benötigten Daten auch richtig und vollständig gemessen werden. Bitte denken Sie nach, ob die gemessenen Werte sinnvoll sind!

Während der Versuchsdurchführung ist ein Messprotokoll dokumentenecht anzufertigen. Es darf also nur Kugelschreiber oder Tusche verwendet werden (kein Bleistift). Es wird nichts radiert, sondern nur gestrichen. Datum und Mitarbeiter angeben, Seiten nummerieren. Die Versuchsdurchführung muss nachvollziehbar sein. Darauf müssen folgende Informationen zu finden

\footnotetext{
1 Abweichende Absprachen mit dem jeweiligen Betreuer bei Zustimmung der Praktikumsleitung sind möglich, jedoch keine Arbeiten außerhalb der normalen Arbeitszeit (d.h. nicht vor 8 Uhr und nicht nach 18 Uhr, nicht am Wochenende).

2 Bitte den Assistenten darauf hinweisen, dass dies auch auf der Karteikarte vermerkt wird.
} 
sein: - Name des Versuchs - Datum der Durchführung - Namen aller beteiligten Praktikanten

- Nummer der verwendeten Apparatur (1, 2, 3 oder A, B, C, sofern vorhanden) - die gemessenen Werte mit Fehlerangabe. Das Protokoll muss leserlich sein und sollte übersichtlich gestaltet sein, z.B. durch einleitende Sätze, was bei den dann folgenden Messwerten bestimmt werden soll. Jeder Messwert muss eindeutig mit der gemessenen Größe in Verbindung gebracht werden können, ggf. sollten Skizzen (ein Bild sagt mehr als 1000 Worte) angefertigt werden. Es müssen die tatsächlich gemessenen (direkt abgelesenen) Werte aufgeschrieben werden, zusätzlich ausgerechnete Werte (z.B. Differenzen) dürfen nur zusätzlich aufgeschrieben werden. Dies soll (Kopf-)Rechen- und Denkfehlern vorbeugen. Zu jedem Messwert ist die Einheit zu notieren! $\mathrm{Zu}$ jedem Messwert ist ein Fehler zu notieren (Ablesefehler, Gerätefehler, Schwankungen).

Wichtig: Am Ende des Versuchs muss das Messprotokoll vom Assistenten testiert werden, sonst ist es ungültig! Sinnigerweise sollte der Versuch erst hiernach abgebaut werden, da u.U. bestimmte Dinge erneut gemessen werden müssen oder Daten fehlen. Grundsätzlich braucht jede Praktikantin ein vom Assistenten original unterschriebenes Messprotokoll. Dieses Protokoll kann aber auch nach dem Versuch von dem/der Gruppenpartner/in kopiert werden. Die Kopie muss dann am gleichen Tag vom Assistenten durch Unterschrift bestätigt werden. Das Protokoll muss nach dem Versuch kopiert und testiert werden. Jede(r) Student/in muss ein eigenes testiertes Messprotokoll haben. Erst nachdem der/die Betreuer/in die Werte kontrolliert, das Versuchsprotokoll testiert (Versuchs-Testat) und dies in die Karteikarte eingetragen hat, ist der Versuch abzubauen und alles aufzuräumen. Die einzige Ausnahme stellen die Versuche mit Computergesteuerter Datennahme da, also Interferenz von Laserlicht, Röntgenstrahlung, Zeeman-Effekt und Radioaktivität: Sofern unmittelbar nach dem Versuch dem/der Betreuer(in) die Daten verfügbar gemacht werden (per USB-Stick, Email, etc.) kann der Messwerte-Teil des testierten Messprotokolls entfallen. Notizen zum Versuchsverlauf sollten natürlich trotzdem angefertigt werden.

Nach Beendigung eines Versuchstages sind alle Versuche, Geräte und Räume wieder in den ursprünglichen Zustand zu versetzen. Messgeräte, Kabel und Stoppuhren sind wieder an die vorgesehenen Stellen zu bringen. Defekte sollen sofort einem/r Betreuer/in gemeldet werden. Flaschen und sonstige Abfälle sind bitte zu entsorgen.

\section{B.5 Protokolle}

Beachten Sie zum Protokoll auch die Hinweise in Kapitel G des 1. Bands der Praktikumsanleitung [26]. Im Protokollkopf müssen der Name des Versuchs und das Datum der Durchführung stehen. Bei einem in Eigenarbeit geschriebenen Protokoll steht als »Praktikant« der Name des Praktikanten und unter »Mitarbeiter « die Namen der übrigen an der Versuchsdurchführung beteiligten Personen. Bitte auch den Namen des/der Betreuers/in und die eigene E-Mail Adresse im Kopf angeben. Wurde das Protokoll in Gruppenarbeit erstellt, stehen alle Namen als »Praktikanten « auf dem Protokoll. Im letzteren Fall liegt es, wie auch beim Messprotokoll, im Ermessen des Betreuers, nur ein einziges Exemplar zu fordern, das nach dem Testat von den Studenten kopiert wird. Diese Kopie wird dann vom Betreuer durch Unterschrift bestätigt und gilt als Originaldokument. Jeder Praktikant braucht für die Unterschrift auf der Karteikarte eine eigene Kopie des Protokolls, welches auch unterschrieben werden soll. Das Erstellen von Protokollen ist nur im Rahmen der Gruppe, mit der auch die Versuchsdurchführung stattfand, gestattet, d.h. die Gruppe sollte maximal zwei, in Ausnahmefällen drei, Personen umfassen. 


\section{B.5.1 Vollversion eines Protokolls}

Das Protokoll muss leserlich und übersichtlich gestaltet sein. Es ist für sich eigenständig, also keine Verweise auf die Praktikumsanleitung $3 \mathrm{Zu}$ Anfang sollen in einem kurzen theoretischen Teil die wesentlichen physikalischen Grundlagen und Formeln erläutert werden. Bei Formeln müssen alle Variablen benannt bzw. definiert werden. Zeichnungen und Skizzen dürfen so oft wie möglich eingefügt werden. Danach sollte kurz beschrieben werden, wie die Auswertung aufgebaut ist, d.h. welche Werte gemessen wurden und was man aus diesen Messwerten bestimmen möchte.

Der Theorieteil sollte kurz und prägnant sein, aber alles Wichtige enthalten. Die Betreuerinnen und Betreuer sind angehalten überlange Theorieteile (mit zu viel Prosa) mit der Bitte um Kürzung zurückzugeben.

Im eigentlichen Teil der Auswertung sind deutlich und nachvollziehbar die einzelnen Auswertungsschritte aufzuschreiben. Einleitende Sätze, was gemessen wurde, und was daraus berechnet wird, sind obligatorisch. Ergebnisse sind deutlich zu kennzeichnen (Rahmen, farbiges Markieren, größere Schrift, usw.). Was sind Zwischen- oder Hilfsergebnisse, was sind Endergebnisse? Alle benutzten Formeln müssen im theoretischen Teil beschrieben sein bzw. aus den dort vorgestellten Formeln hergeleitet werden. Es ist auf eine durchgehend eindeutige Variablendefinition und Variablenbenutzung im Protokoll zu achten. Alle Variablen in den Funktionen sind zu benennen bzw. zu definieren. Die Fehlerrechnung muss nachvollziehbar beschrieben werden (einfacher Mittelwert oder gewichtetes Mittel, ggf. Formel der Fehlerfortpflanzung angeben). Fehler sind sinnvoll anzugeben! Alle Werte haben Einheiten, alle Grafen eine Beschriftung! Bei Vergleich mit Literaturwerten: Woher kommen die Werte (Quellenangabe)? Eine Diskussion der Ergebnisse und der Fehler ist obligatorisch. Dazu muss man sich natürlich vorher die Frage stellen, ob das, was man berechnet hat, ein sinnvolles Ergebnis ist.

Bei der Abgabe des Protokolls muss das dazugehörige original unterschriebene Messprotokoll mit abgegeben werden. Protokolle müssen in geeigneter Form zusammengeheftet sein (einfache Heftung reicht vollkommen), lose Blätter werden nicht akzeptiert. Wird ein/e Praktikant/in auf die Auswertung seines/ihres eigenen oder des gemeinsam in der Gruppe erstellten Protokolls angesprochen und kann keine Auskunft zu den gemachten Rechnungen geben, so gilt das Protokoll als nicht selbständig erstellt und wird nicht testiert. Protokolle mit einer Auswertung, die nicht auf den eigenen Messdaten basieren, bei denen die Messdaten nachträglich geändert wurden oder bei denen die Liste der am Versuch beteiligten Personen erweitert wurde, gelten als Täuschungsversuch/Urkundenfälschung und werden entsprechend geahndet.

\section{B.5.2 Reduzierte Version}

Bei einzelnen Protokollen (s. Abschnitt B.5.4) ist eine Reduktion der niedergeschriebenen Rechnungen vorgesehen, um den Aufwand der Anfertigung zu begrenzen. Dabei sollen natürlich die durchgeführten Rechnungen entsprechend der oben aufgeführten Qualitätskriterien ausgeführt werden; lediglich deren detaillierte Angabe im Protokoll wird reduziert. Im Protokoll muss in jedem Fall die abschließende Formel eines Auswertungsschritts vorhanden sein, nur Zwischenschritte können entfallen. Die Details der zugehörigen Fehlerrechnung können ebenfalls entfallen, wobei der abschließender Wert angegeben bzw. in den entsprechenden Graphen eingezeich-

3 Sätze wie »Versuchsdurchführung s. Praktikumsanleitung« sind überflüssig. 
net werden muss und die eingehende Größen und deren Wert nachvollziehbar bleiben müssen. Die Kriterien zur graphischen Darstellungen bleiben unberührt.

\section{B.5.3 Experimenteller Schwerpunkt}

Ebenfalls bei ausgewählten Versuchen (s. Abschnitt B.5.4) soll der Schwerpunkt auf experimentellen Aspekten des Versuchs und auf der Auswertung liegen. Theorie und die detaillierte Beschreibung der Durchführung entfallen, Auswertung inklusive Fehlerrechnung werden wie in Anleitung angegeben bearbeitet und wie in Abschnitt B.5.2 protokolliert. Stattdessen wird der Abschnitt zur Diskussion um ein ausführlicheres Hinterfragen der verwendeten Methode erweitert, um zu beantworten, warum so wie angegeben gemessen und ausgewertet wird. Es empfiehlt sich, dies innerhalb der Messgruppe vor Anfertigung des Protokolls zu diskutieren. Das Protokoll sollte damit eher im Stil einer Hausaufgabenrechnung (auf Wunsch handschriftlich) angefertigt werden.

\section{B.5.4 Regeln}

Im Praktikum zu Experimentalphysik III wird das benotete Protokoll und das Protokoll eines weiteren Versuchs nach Wahl (außer den beiden gemäß Abschnitt B.5.3) in Vollversion erstellt. Außerdem werden zu je zwei Versuchen, die während der Vorbesprechung von der Praktikumsleitung festgelegt werden, Protokolle wie in Abschnitt B.5.3 angegeben und alle übrige Protokolle entsprechend Abschnitt B.5.2 angefertigt.

Ein vollständiges Protokoll muss innerhalb einer Woche abgegeben werden. Wird das Protokoll nicht innerhalb von 1 Woche (gerechnet vom Tag der Versuchsdurchführung) abgegeben, wird das Protokoll nicht mehr angenommen und der Versuch gilt als nicht durchgeführt. Pro Praktikumsabschnitt kann die Abgabefrist bei entsprechender Begründung in Abstimmung mit dem Betreuer bei einem Versuch auf zwei Wochen verlängert werden.

Ein Protokoll gilt nur dann als vollständig, wenn es die oben genannten Bedingungen erfüllt. Insbesondere gilt es als nicht vollständig, wenn es außer dem Endergebnis keine Zwischenergebnisse enthält, die den Rechenweg und die Werte nachvollziehbar machen. Sollte das Protokoll für Korrekturen ohne Testat zurückgegeben werden, so gilt erneut die 1 Woche-Frist ab dem Tag der Rückgabe. Die Korrekturen sind (z.B. als Anhang) zusammen mit dem vollständigen ursprünglichen Protokoll abzugeben. Es ist zu beachten, dass es für jedes Semester einen Termin gibt, ab dem alle bis zu diesem Tag nicht testierten Protokolle nicht mehr angenommen und testiert werden. Dies ist in der Regel der 31.03. für das vergangene Wintersemester und der 31.09. für das vergangene Sommersemester. Dies ist erforderlich, da auch die Betreuer Fluktuationen unterworfen sind und so der/die Betreuer/in später eventuell Göttingen schon verlassen hat.

Auch für die Rückgabe der Protokolle durch die Assistenten soll die 1-Woche-Frist eingehalten werden 4 Bei Angabe der E-Mail Adresse auf dem Protokoll kann der/die Betreuer/in eine Nachricht schicken, wenn die Korrektur fertig ist.

4 Sollten hier drastische Verzögerungen auftreten, benachrichtigen Sie bitte die Praktikumsleitung. 


\section{B.6 Nachholtermine}

Nach jedem Versuchsblock stehen Nachholtermine für versäumte Versuche zur Verfügung. Bitte sorgen Sie dafür, dass ggf. ein/e Partner/in für die Versuchsdurchführung zur Verfügung steht.

\section{B.7 Benotung}

Es wird im dritten Praktikumsabschnitt (B.Phy.1103.2) eins der sieben Protokolle zur Benotung herangezogen, d.h. die Benotung wird während des Praktikums vorgenommen. Der vierte Praktikumsabschnitt (B.Phy.1104.2) ist unbenotet.

Die Auswahl der zu benotenden Protokolle geschieht wie folgt: Pro Themenblock wird aus zwei möglichen Versuchen vor Abgabe ein Versuch gewählt, zu dem in vollkommener Einzelarbeit, also nicht wie bei den übrigen Versuchen zu zweit, das Protokoll angefertig 5 und zur Bewertung spätestens zwei Wochen nach dem Versuchstermin bei der Praktikumsleitung abgegeben wird. Diese benoteten Protokolle werden also nicht wie die übrigen Protokolle bei Mängeln zur Verbesserung zurückgegeben, sondern nach Korrektur und Benotung als Prüfungsleistung archiviert. Es empfiehlt sich daher, zunächst einige Protokolle ohne Benotung anzufertigen. Die endgültige Benotung geschieht durch die Praktikumsleitung. Zur Auswahl stehen dabei die folgenden Versuche:

1. Wellen und Optik (3. Teil, B.Phy.1103.2):

- Prismen- und Gitterspektrometer

- Fresnelsche Formeln und Polarisation

2. Atomphysik (4. Teil, B.Phy.1104.2): keine Benotung

\section{B.8 Karteikarte}

Nach der Anmeldung zum Praktikum werden zum ersten Versuch Karteikarten ausgeteilt, die die Praktikantin oder der Praktikant bis zum Abschluss des jeweiligen Praktikumsabschnitts behält. In diese werden dann die Versuchs-Durchführung, die Theorievorträge und alle Protokoll-Testate vom Assistenten eingetragen und mit Unterschrift bestätigt. Diese Karteikarte ist der Nachweis für die Gesamtleistung im Praktikum und damit Zulassung zum Eintrag in FlexNow, also bitte nicht verlieren.

Nach Abschluss des Praktikums geben Sie bitte Ihre vollständig ausgefüllte Karteikarte bei der Praktikumsleitung ab, damit Sie Ihre Zulassungsberechtigung (s.u.) erhalten (Abgabe im Postfach genügt).

\section{B.9 Leistungsnachweis}

Die Gesamtleistung besteht damit im dritten Teil (B.Phy.1103.2) aus sieben durchgeführten Versuchen mit einem benoteten und sechs weiteren (unbenoteten, aber testierten) Protokollen und

5 Die beiden Praktikumspartner können sich dabei für unterschiedliche Versuche entscheiden. Als Konsequenz muss aber auch der/die Partner/in, dessen/deren Protokoll zum gewählten Versuch nicht bewertet wird, dieses in Einzelarbeit anfertigen, was also insgesamt mehr Arbeit bedeutet. 
im vierten Teil (B.Phy.1104.2) aus sieben durchgeführten Versuchen mit sieben testierten Protokollen und je einem Theorievortrag. 


\section{Sicherheit im Praktikum}

Nehmen Sie Ihre eigene Sicherheit und die Ihrer Kommilitonen sehr wichtig. Auch im Praktikum gibt es viele Gefahrenquellen (Spannung, Strom, Wasserdampf, Kochplatten, flüssiger Stickstoff, Radioaktivität, Gase, Druck, Vakuum, et cetera). Bitte machen Sie sich dies immer bewusst und handeln Sie besonnen. Immer zuerst denken, dann handeln. Sind Sie sich über Gefahren, Prozeduren und Vorgehensweisen im Unklaren, wenden Sie sich bitte zuerst an eine betreuende Person. Generell sind alle Unfallverhütungsvorschriften UVV zu beachten.

Aus Sicherheitsgründen müssen während des Aufenthaltes in den Räumen des Praktikums mindestens zwei Studierende anwesend und eine betreuende Person in unmittelbarer Nähe sein, damit bei einem Unfall für eine schnelle und wirksame Erste Hilfe gesorgt werden kann. Für dringende Notfälle sind bei den Telefonen die Notrufnummern 110 und 112 freigeschaltet.

Folgende Sicherheitsbestimmungen fassen die für das Praktikum wichtigsten Punkte zusammen und erheben keinen Anspruch auf Vollständigkeit. Auf Wunsch können die einschlägigen Sicherheitsbestimmungen eingesehen werden.

In den Labors und Praktikumsräumen darf weder geraucht noch gegessen oder getrunken werden.

Im Falle eines Feuers ist unverzüglich eine betreuende Person zu verständigen. Feuerlöscher befinden sich in den Fluren. Die Feuerwehr ist unter der Notrufnummer 112 zu erreichen. Die Feuermelder sind im Notfall auf dem Weg aus dem Gebäude zu betätigen. Bei einem Feueralarm ist das Gebäude auf den gekennzeichneten Fluchtwegen zügig, aber ruhig zu verlassen. Man muss sich am entsprechenden Sammelpunkt vor dem Gebäude einfinden (Mitte des Parkplatzes), damit festgestellt werden kann, ob alle Personen das Praktikum verlassen haben. Es gilt der generelle Grundsatz »Personenschutz geht vor Sachschutz $«$.

Auch bei Unfällen oder Verletzungen ist sofort eine betreuende Person zu benachrichtigen. Ein Verbandskasten ist in den Praktikumsräumen vorhanden. Die Notrufnummern 110 und 112 sind freigeschaltet. Ein Notfallblatt mit entsprechenden Telefonnummern ist an verschiedenen Stellen ausgehängt. Die betreuende Person muss Unfälle und Verletzungen sofort weitermelden.

Werden Schäden an einer Apparatur oder an einem Gerät festgestellt, dürfen diese nicht weiter verwendet werden. Bitte sofort eine betreuende Person benachrichtigen.

Bananenstecker gehören keinesfalls in Steckdosen! Bei Aufbau und Arbeiten an elektrischen Schaltungen ist die Schaltung zuerst in einen spannungsfreien Zustand zu bringen. Schaltungen sind vor deren Einsatz durch eine betreuende Person zu kontrollieren. Schwingkreise, Spulen und Kondensatoren können auch nach Abschalten der Spannung noch eine längere Zeit Spannung führen. Sollte ein elektrischer Unfall passieren, ist sofort der NOT-AUS Schalterl zu betätigen und dann Hilfe zu leisten. Danach sofort eine betreuende Person verständigen oder weitere Hilfe veranlassen.

Beim Umgang mit Chemikalien und anderen Gefahrstoffen sind die Gefahrstoffverordnung und weitere Vorschriften zu beachten. Beim Umgang mit radioaktiven Stoffen und ionisierender

1 Der NOT-AUS Schalter (Roter Knopf auf gelbem Grund), welcher den ganzen Raum stromlos schaltet, befindet sich immer direkt neben der Raumtür. Bei Gefahr einfach eindrücken. 
Strahlung ist die Strahlenschutzverordnung (StrSchV) und Röntgenverordnung (RöV) zu beachten. Beide liegen in den jeweiligen Räumen aus. Da diese Gesetze auch besondere Regeln für Schwangere enthalten, müssen Schwangerschaften dem Praktikumsleiter gemeldet werden. Am Versuch »Radioaktivität« darf dann nicht teilgenommen werden.

Kochendes Wasser, Wasserdampf und Heizplatten stellen ein Gefahrenpotenzial für schwere Verbrennungen dar. Unter Druck stehender Wasserdampf (Versuch »Dampfdruck «) ist noch eine Stufe gefährlicher.

Laserlicht ist äußerst intensiv und kann bei direkter Einstrahlung in das Auge zu Schädigungen, bis hin zur Erblindung, führen. Im Praktikum werden Laser der Klasse 2 oder Klasse 3 verwendet. Gehen Sie bitte entsprechend vorsichtig damit um.

Druckgasflaschen stehen unter sehr hohem Druck, sie sind nur durch eine betreuende Person zu benutzen. Die Betätigung der zentralen Gasarmaturen in den Praktikumsräumen erfolgt ausschließlich durch den Praktikumstechniker.

Die Betreuerinnen und Betreuer sind gehalten, vor jedem Versuch nochmals die Sicherheitsaspekte zum Versuch zu erläutern und deren Verständnis zu überprüfen.

Generell gilt: Alle Unfälle und Verletzungen sind sofort einer betreuenden Person zu melden, die dann das weitere veranlassen und den Unfall weitermelden muss. 



\section{Teil II}

\section{Versuche}




\section{Ultraschallwellen}

Anhand des Schalldurchgangs in einer Platte unter verschiedenen Winkeln soll die Entstehung und Transmission von longitudinalen und transversalen Schallwellen in einem Festkörper gemessen werden. Die Schallgeschwindigkeit beider Wellenarten kann aus der Beziehung zwischen Amplitude und Einfallswinkel bestimmt werden.

\subsection{Stichworte}

Schallwellen, Wellenausbreitung, longitudinale und transversale Wellen, Ultraschallwellen im Festkörper, Reflexion und Transmission, Brechung.

\subsection{Literatur}

Empfohlen: Dem 1 [11]: Kap. 6.2, 11.9.4, 11.9.5 und 11.11.4.

\subsection{Zubehör}

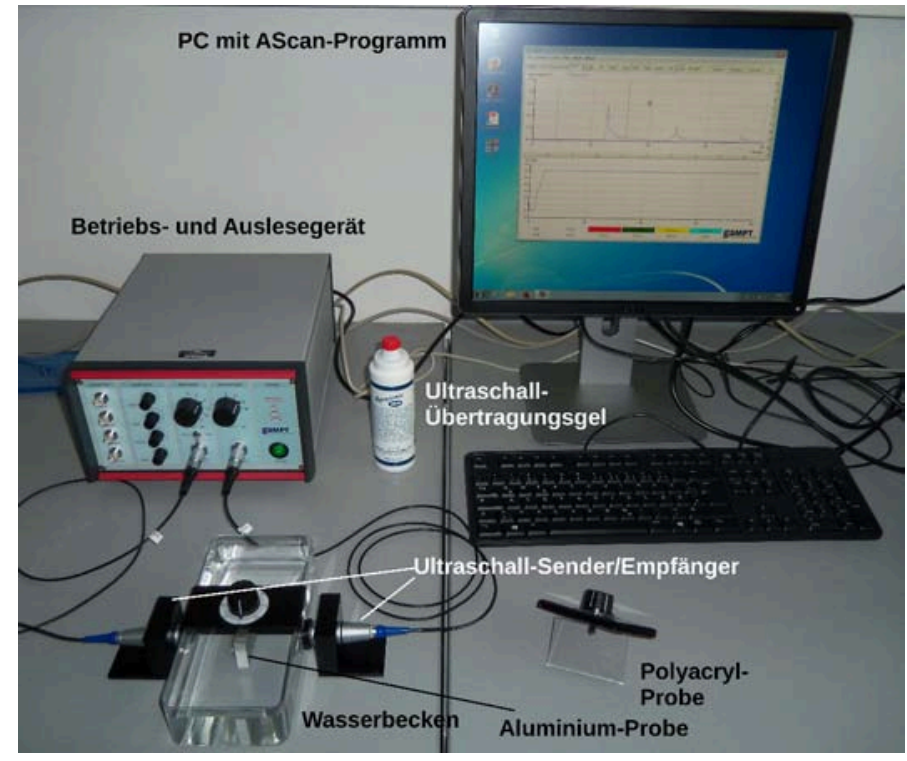

Bild 12.1: Der Versuch $»$ Ultraschall«.

Bild 12.1 zeigt ein Foto des Versuchsaufbaus mit Zubehör: zwei Ultraschallsonden mit Betriebsund Auslesegerät, ein Probenhalter mit Winkelskala und Aluminium- und Polyacryl-Probe, ein 
Wasserbecken zum Einkoppeln der Ultraschallwellen, Ultraschall-Übertragungsgel, PC zur Bedienung des Betriebsgeräts.

\subsection{Grundlagen}

Ebene Wellen können grundsätzlich longitudinal oder transversal auftreten, d.h. mit oder senkrecht zu der Ausbreitungsrichtung schwingen. Im Gegensatz zu elektromagnetischen Wellen, die nur transversaler Natur sind (s. Kapitel 17), können Schallwellen in einem Festkörper in beiden Versionen auftreten.

Trifft eine Schallwelle senkrecht auf die Oberfläche eines Festkörpers, so bildet sich eine longitudinale Welle über die elastische Spannung $\sigma$ aus. Diese ist mit einer relativen Dehnung $\varepsilon$ über das Elastizitätsmodul $E$ verknüpft:

$$
\sigma=E \varepsilon
$$

Die elastische Spannung sorgt für eine rückstellende Kraft, welche die Masse des verschobenen Bereichs beschleunigt, so dass sich insgesamt eine Wellengleichung ergibt:

$$
\frac{\partial^{2} \xi}{\partial t^{2}}=\frac{E}{\rho} \frac{\partial^{2} \xi}{\partial z^{2}} .
$$

Dabei sind $\xi$ die Auslenkung, $\rho$ die Dichte des Materials und $z, t$ die Orts- bzw. Zeitkoordinate der Wellenausbreitung. Die longitudinale Ausbreitungsgeschwindigkeit $v_{L}$ ist damit durch

$$
v_{L}=\sqrt{\frac{E}{\rho}},
$$

gegeben. Genau genommen muss zusätzlich eine Querkontraktion berücksichtigt werden. Dies führt zu einer Korrektur von Gl. 12.3) mittels der Poissonzahl $\mu$ :

$$
v_{L}=\sqrt{\frac{E(1-\mu)}{\rho(1+\mu)(1-2 \mu)}} .
$$

Trifft eine Schallwelle hingegen unter einem Winkel auf einen Festkörper auf, werden durch Scherkräfte Transversalwellen angeregt. Die Wellengleichung ergibt sich analog zur Longitudinalwelle, wobei das Elastizitätsmodul durch das Torsionsmodul $G$ ersetzt wird. Damit wird die transversale Ausbreitungsgeschwindigkeit $v_{T} \mathrm{zu}$

$$
v_{T}=\sqrt{\frac{G}{\rho}} .
$$

Die Transmission der Transversalwelle ist am größten bei einem Durchgangswinkel von $\beta=$ $45^{\circ}$. Dies entspricht damit dem Winkel nach der Brechung an der Grenzfläche. Nach dem Snelli- 
usschen Brechungsgesetz gilt somit für den Einfallswinkel $\alpha$

$$
\frac{v_{T}}{v_{F}}=\frac{\sin \beta}{\sin \alpha} .
$$

Hierbei ist $v_{F}$ die Schallgeschwindigkeit der den Festkörper umgebenden Flüssigkeit. Damit wird der Winkel $\alpha_{\max }$, bei dem die Amplitude der transmittierten Welle maximal wird, zu

$$
\sin \alpha_{\max }=\frac{v_{F}}{v_{T}} \sin 45^{\circ}=\frac{v_{F}}{\sqrt{2} v_{T}} .
$$

Beide Wellentypen verschwinden bei der Transmissionsmessung für die Totalreflexion, d.h. bei $\beta=90^{\circ}$, so dass der zugehörige Einfallswinkel $\alpha_{\text {tot }} \mathrm{zu}$

$$
\sin \alpha_{\mathrm{tot}}=\frac{v_{F}}{v_{L}, T} \sin 90^{\circ}=\frac{v_{F}}{v_{L}, T}
$$

wird.

\subsection{Fragen}

1. Was sind longitudinale und transversale Wellen?

2. Was ist das Elastizitätsmodul, was die Poissonzahl?

3. Was ist das Torsionsmodul?

4. Nennen und erläutern Sie eine Anwendung für Laufzeitmessungen von Ultraschallsignalen.

\subsection{Durchführung}

Schließen Sie die beiden Ultraschallsonden an den RECEIVER bzw. TRANSMITTER-Ausgang des Betriebsgeräts an. Füllen Sie das Plexiglasbecken mit Wasser, so dass die beiden an der Längsseite außen angebrachten Sonden vollständig bedeckt sind (für die Anbringung das UltraschallÜbertragungsgel verwenden). Auf dem PC als Benutzer prakt einloggen (kein Passwort), das Betriebsgerät anschalten und auf dem PC das Programm AScan starten. Im oberen Teil der Anzeige ist der Zeitverlauf der Amplitude des empfangenen Signals zu sehen, im unteren der Zeitverlauf der Verstärkung, die nur während des Sendesignals $(t=0)$ klein und ansonsten möglichst groß und zeitlich flach sein sollte: mit den Knöpfen unter TIMER-TGC am Betriebsgerät einstellen. Unter RECEIVER kann zwischen REFLECT . und TRANS ., also reflektiertem bzw. transmittiertem Signal an Hand der Laufzeit unterschieden werden. Auf transmittiertes Signal stellen, Verstärkung unter RECEIVER auf kleinsten Wert setzen und Ausgangsleistung unter TRANSMITTER so einstellen, dass das empfangene Signal gut erkennbar ist und unter 1,2 V liegt, da ansonsten der Verstärker in Sättigung geht. Ggf. Position der Sonden justieren. In der Menüleiste des Programms auf die volle Zeitachse stellen (der Knopf neben Stop sollte Half anzeigen, ansonsten Full drücken).

Eine Bedienungsanleitung des Steuergeräts befindet sich auf dem Desktop unter dem Icon AScan Handbuch.

1. Platzieren Sie die Polyacrylplatte in die Mitte des Beckens und stellen den Knopf auf $20^{\circ}$. 
Es sollte nun zwei Signale, ggf. mit einem «Satelliten» zu sehen sein (durch Mehrfachreflexion, diese ignorieren): das frühere Signal entspricht wegen der etwa zweifach größeren Schallgeschwindigkeit der longitudinalen Schallwelle, das spätere entsprechend der transversalen Welle. Messen Sie die Amplitude beider Signale mit dem hellblauen Cursor im AScan-Programm als Funktion des Auftreffwinkels in Schritten von 2,5 $5^{\circ}$ zwischen $0^{\circ}$ und $90^{\circ}$; ggf. während der Messung die Verstärkung erhöhen.

2. Wiederholen Sie diese Messung analog mit der Aluminiumplatte.

\subsection{Angaben}

Tabelle 12.1 enthält Angaben zu den verwendeten Festkörpern. Die Schallgeschwindigkeit in Wasser beträgt $v_{W}=1480 \mathrm{~m} / \mathrm{s}$ [66].

Tabelle 12.1: Elastische Koeffizienten und Dichte der verwendeten Materialien [66].

\begin{tabular}{lll}
\hline Größe & Polyacryl & Aluminium \\
\hline$\rho\left[\mathrm{g} / \mathrm{cm}^{3}\right]$ & 1,19 & 2,7 \\
$G[\mathrm{MPa}]$ & 1700 & 27000 \\
$E[\mathrm{MPa}]$ & 3300 & 72000 \\
\hline
\end{tabular}

\subsection{Auswertung}

1. Tragen Sie jeweils die Amplitude der longitudinalen und der transversalen Welle gegen den Winkel auf. Bestimmen Sie den Winkel des ersten Verschwindens der Welle. Dieser entspricht dem Winkel für Einsetzen der Totalreflexion. Berechnen Sie daraus die entsprechende longitudinale und transversale Schallgeschwindigkeit in beiden Festkörpern.

2. Bestimmen Sie das Maximum der Amplitude des transversalen Signals und berechnen Sie hieraus ebenfalls die transversale Schallgeschwindigkeit.

3. Vergleichen Sie beide Messergebnisse zur transversalen Schallgeschwindigkeit und beide Schallgeschwindigkeiten mit den entsprechenden Literaturwerten.

\subsection{Bemerkungen}

Der Verstärker verwendet die Einheit Dezibel (dB). Diese gibt die Verstärkung $v$ der Eingangsspannung $V_{i}$ zur Ausgangsspannung $V_{a}$ logarithmisch an:

$$
v[\text { in } \mathrm{dB}]=10 \log \frac{V_{a}^{2}}{V_{i}^{2}}
$$




\section{Mikrowellen}

In diesem Versuch werden Mikrowellen mit einem Gunn-Oszillator generiert, aus denen auf zwei verschiedene Arten eine stehende Welle erzeugt wird. Deren Feldverlauf wird mittels einer E-Feld-Sonde vermessen, um die Wellenlänge in Luft und in einem dielektrischen Medium zu bestimmen.

\subsection{Stichworte}

Elektromagnetische Wellen, Ausbreitung, stehende Wellen, Lecherleitung, dielektrische Medien.

\subsection{Literatur}

Empfohlen: Dem 2 [12]: Kap. 7.8 und 7.9; Gerthsen [45]: Kap. 8.4.3, 8.4.9, 8.4.10.

\subsection{Zubehör}
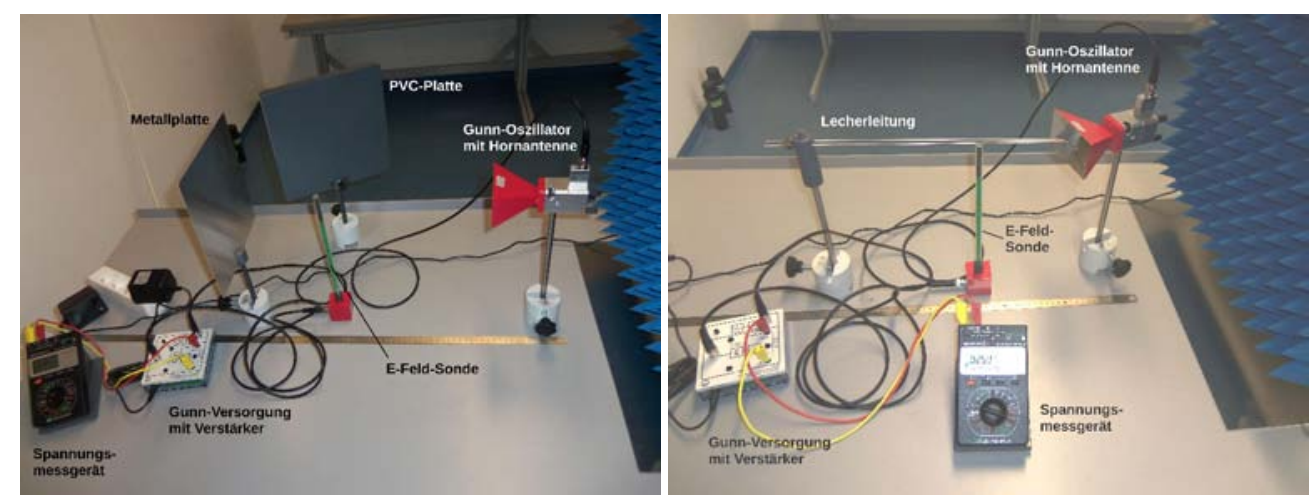

Bild 13.1: Die Versuchsteile »Wellenlängenbestimmung an stehenden Mikrowellen« (links) und »Mikrowellen in einer Lecherleitung « (rechts).

Bild 13.1 zeigt links ein Foto des Versuchsteils »Wellenlängenbestimmung an stehenden Mikrowellen« mit Zubehör: Gunn-Versorgung mit Verstärker, Gunn-Oszillator mit Hornantenne, E-Feld-Sonde, Spannungsmessgerät, Maßstab, Metall- und PVC-Platte und rechts ein Foto des 
Versuchsteils »Mikrowellen in einer Lecherleitung « mit Zubehör: Gunn-Versorgung mit Verstärker, Gunn-Oszillator mit Hornantenne, E-Feld-Sonde, Spannungsmessgerät, Maßstab, Lecherleitung.

\subsection{Grundlagen}

Sowohl zur Mikrowellen-Erzeugung als auch zur -Messung werden in diesem Versuch stehende Wellen verwendet. Diese bilden sich aus, wenn sich durch Reflexion erzeugte gegenläufige Wellen so überlagern, dass die Amplitude der resultierenden Welle ortsfest ist.

Betrachten wir das elektrische Feld einer ebenen elektromagnetischen Welle der Wellenlänge $\lambda$ und Amplitude $\vec{E}_{0}$, die sich in $z$-Richtung ausbreitet, d.h.

$$
\vec{E}(z, t)=\overrightarrow{E_{0}} \cdot \cos \left(\frac{2 \pi c}{\lambda} t-\frac{2 \pi}{\lambda} z\right) .
$$

Dabei bezeichnet $c$ die Lichtgeschwindigkeit. Eine leitende Platte befinde sich bei $z=0$ senkrecht zu $\vec{e}_{z}$, d.h. die transversalen Komponenten von $\vec{E}$ können dort wegen der Leitfähigkeit nicht existieren. Sind $\vec{E}_{i}$ und $\vec{E}_{r}$ die Amplituden der Feldstärken der ein- und auslaufenden Wellen, so muss

$$
\left(\vec{E}_{i}+\vec{E}_{r}\right)(z=0)=\overrightarrow{0} \Rightarrow \vec{E}_{i}=-\vec{E}_{r}:=\vec{E}_{0}
$$

gelten. Damit ergibt sich für die gesamte stehende Welle

$$
\begin{aligned}
\vec{E}(z, t) & =\vec{E}_{i} \cos \left(\frac{2 \pi c}{\lambda} t-\frac{2 \pi}{\lambda} z\right)+\vec{E}_{r} \cos \left(\frac{2 \pi c}{\lambda} t+\frac{2 \pi}{\lambda} z\right) \\
& =2 \vec{E}_{0} \sin \left(\frac{2 \pi c}{\lambda} t\right) \sin \left(\frac{2 \pi}{\lambda} z\right)
\end{aligned}
$$

so dass die Wellenlänge $\lambda$ direkt die Ortsabhängigkeit der Amplitude der stehenden Welle beschreibt.

Dreidimensionale stehende elektromagnetische Wellen können sich in einem sogenannten Hohlraumresonator ausbilden. In diesem Versuch wird ein solcher Hohlraumresonator mittels eines Gunn-Oszillators zu Schwingungen im Mikrowellenbereich angeregt, d.h. zu elektromagnetischen Wellen im Frequenzbereich $300 \mathrm{MHz}$ bis $300 \mathrm{GHz}$. Um in einem rechteckigen Hohlraumresonator mit den Kantenlängen $a, b$ und $c$ stehende Wellen der Frequenz $f$ anzuregen, muss die Bedingung

$$
f=\frac{c}{2} \sqrt{\frac{m}{a^{2}}+\frac{n}{b^{2}}+\frac{q}{c^{2}}}
$$

mit ganzen Zahlen $m, n$ und $q$ erfüllt sein. Die niedrigste Frequenz ergibt sich also für $m=n=$ $q=1$.

Treffen die so erzeugten Mikrowellen auf eine sich senkrecht zur Abstrahlrichtung stehende leitende Platte, bildet sich wiederum wie oben beschrieben eine stehende Welle aus, deren Eigenschaften vermessen werden können. Alternativ können die Mikrowellen in einen Wellenleiter eingespeist werden. Verwendet man zwei parallele, am Ende kurzgeschlossene Leiter, eine 
sog. Lecherleitung, so bildet sich auf dieser eine periodische Spannungs- und Stromverteilung aus, deren Frequenz und Wellenlänge der ursprünglichen Mikrowelle entsprechen. Der Kurzschluss am Ende der Leitung agiert dabei analog zur Metallplatte.

\subsection{Fragen}

1. Warum muss der gesamte elektrische Feldvektor an der Oberfläche einer senkrecht zur Ausbreitungsrichtung stehenden leitenden Platte verschwinden? Was passiert bei einem anderen Auftreffwinkel?

2. Wie groß ist der Abstand zwischen zwei Wellenknoten einer stehenden Welle der Wellenlänge $\lambda$ ?

3. Wie verhält sich das Magnetfeld einer stehenden Welle im Vergleich zum elektrischen Feld?

\subsection{Angaben}

Die Dicke der PVC-Platte beträgt $20 \mathrm{~cm}$. Die Breite des Hohlraumresonators des Gunn-Oszillators beträgt $(23,0 \pm 0,2) \mathrm{mm}$, wogegen dessen Höhe vernachlässigt werden kann, und der Abstand zwischen Lochblende und Gunn-Element, d.h. die effektive Länge des Hohlraums, beträgt $(22,0 \pm 0,2) \mathrm{mm}$.

\subsection{Durchführung}

Verbinden Sie den oberen Ausgang der Gunn-Versorgung per HF-Kabel mit dem Gunn-Oszillator, die E-Feld-Sonde per HF-Kabel mit dem Verstärkereingang. Das Spannungsmessgerät (auf Wechselspannungsmessung gestellt) mit einfachen Kabeln am AC-Ausgang des Verstärkers anschließen; die angezeigte Spannung ist proportional zur gemessenen Feldstärke.

Stellen Sie nun die E-Feld-Sonde mittig vor die Hornantenne und regeln Sie die Modulationsfrequenz der Mikrowellen mit dem schwarzen Knopf oben links an der Gunn-Versorgung so, dass ein maximales Signal gemessen wird.

1. Bauen Sie den Versuchsteil »Wellenlängenbestimmung an stehenden Mikrowellen« auf: Die Metallplatte auf einem der Halter befestigen und in $200 \mathrm{~mm}$ Abstand und senkrecht zur Abstrahlrichtung der Hornantenne aufstellen.

2. Messen Sie den Feldstärkeverlauf von $50 \mathrm{~mm}$ vor der Hornantenne bis $50 \mathrm{~mm}$ vor der Metallplatte in Schritten von $5 \mathrm{~mm}$. Wählen Sie mindestens je fünf Minima und Maxima in der Feldstärke und messen in deren Umgebung die Feldstärke in kleineren Schritten als $5 \mathrm{~mm}$ aus.

3. Vergrößern Sie den Abstand zwischen Metallplatte und Hornantenne und suchen Sie mit der E-Feld-Sonde ein Minimum in der Feldstärke. Notieren Sie die Position. Bringen Sie nun die graue PVC-Platte auf einem Sockel in den Strahlengang, finden und notieren Sie die verschobene Position des Minimums.

4. Bauen Sie den Versuchsteil »Mikrowellen in einer Lecherleitung« auf: Schieben Sie den Kurzschlussbügel auf die Lecherleitung und stecken Sie diese in die Bohrungen des PVCHalters. Gleichen Sie die Höhen von Hornantenne und Lecherleitung auf das obere Ende 
der E-Feld-Sonde ab. Führen Sie die Induktionsschleife am Ende der Lecherleitung in die Hornantenne ein.

5. Messen Sie den Feldstärkeverlauf über die Länge der Lecherleitung in Schritten von 2 mm.

\subsection{Auswertung}

1. Tragen Sie die Feldstärke aus Messungen2 2 und 5 gegen die Position auf. Bestimmen Sie die Wellenlänge der abgestrahlten Mikrowellen und vergleichen Sie beide Ergebnisse miteinander und mit der aus den Dimensionen des Hohlraumresonators zu erwartenden Wellenlänge.

2. Interpretieren Sie den Feldstärkeverlauf aus Messung 5. Welche Aussagen können Sie über die Ausbreitung entlang der Lecherleitung treffen?

3. Bestimmen Sie den Brechungsindex von PVC aus Messung 3

\subsection{Bemerkungen}

Die Leistung des Gunn-Oszillators ist hinreichend niedrig, um keine Gefahr darzustellen. Trotzdem sollten Arbeiten am Aufbau immer bei ausgeschaltetem Oszillator durchgeführt werden und niemals direkt in die in Betrieb befindliche Hornantenne geblickt werden. 


\section{Das Mikroskop}

Das Mikroskop, erstmals gebaut von Anton van Leuvenhook im Jahr 1673, ist neben dem Fernrohr eines der grundlegendsten Instrumente der geometrischen Optik. Im ersten Teil werden die die Vergrößerung eines Mikroskops beeinflussenden Parameter untersucht und variiert. Im zweiten Teil des Versuches werden das Auflösungsvermögen und die numerische Apertur eines Mikroskops behandelt.

\subsection{Stichworte}

Mikroskop, Auflösungsvermögen, Numerische Apertur, Strahlengang, Abbesche Konstruktion, Immersionsflüssigkeit, Abbildungsmaßstab, Gesamtvergrößerung

\subsection{Literatur}

NPP: 33-36; BS-3: 1.9, 3.9; Gerthsen S. 466ff, Pohl: Optik S. 45ff; Kohlrausch; Walcher; Dem-2.

\subsection{Zubehör}

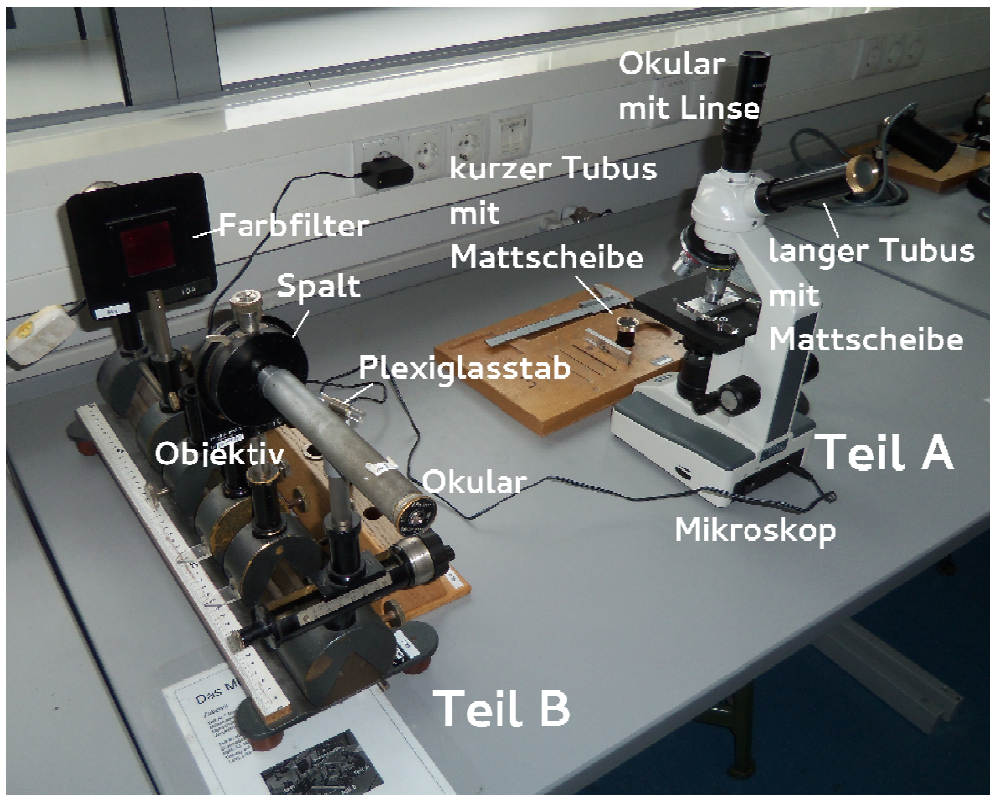

Bild 14.1: Der Versuch »Mikroskop«. 


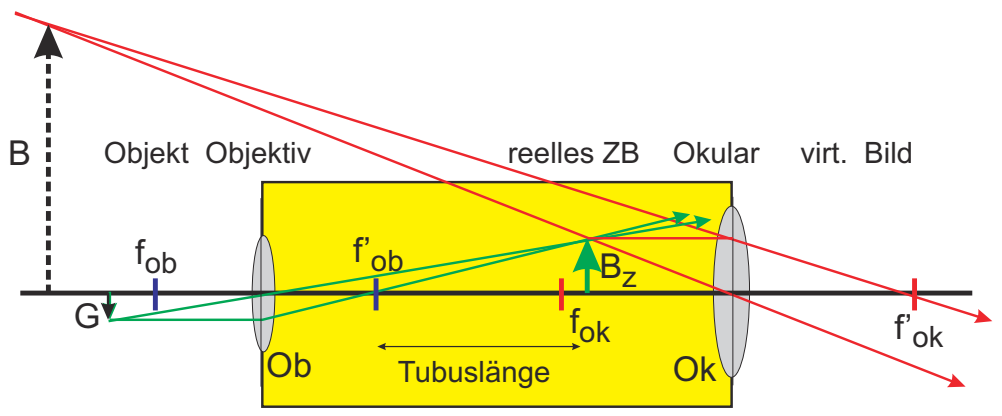

Bild 14.2: Schematischer Strahlengang im Mikroskop.

Bild 14.1 zeigt ein Foto des Versuches mit separatem Zubehör für die beiden Versuchsteile A und $\mathrm{B}$ :

Teil A: 1 Mikroskop mit drei Objektiven (4x, 10x, 40x) und zwei über einen Strahlteiler verbundenen Okularen; 2 verschiebbare Mikroskoptubi mit Mattscheibe; 1 Objektmikrometer, 1 Vergleichsmaßstab.

Teil B: Mikroskop, Präzisionsspalt als Aperturblende, Glasmaßstab, Farbfilter (Wellenlänge $6500 \AA$ A), Mikrometer-Schiebelehre, Plexiglasstab von $5 \mathrm{~cm}$ Länge mit 1/2 mm Teilung auf einer Stirnfläche (Brechungsindex von Plexiglas $n=1,49$ ), Lochblende, Lampe.

\subsection{Grundlagen}

1. Mikroskop:

Mikroskope erzeugen vergrößerte Bilder von kleinen Strukturen, lassen also Objekte unter einem größeren Sehwinkel erscheinen. Im Gegensatz zum Fernrohr liegen die Objekte im Falle des Mikroskops jedoch im Endlichen. Die Vergrößerung wird in zwei Stufen erzeugt. Ein Objektiv erzeugt vom knapp außerhalb der Brennebene liegenden Gegenstand ein reelles Zwischenbild am Ende eines Tubus, welches mit einem lupenartigem Okular beobachtet wird. Der Strahlengang ist in Bild 14.2 schematisch dargestellt.

2. Numerische Apertur und Auflösungsvermögen eines Mikroskops:

Für das Auflösungsvermögen gilt folgende Definition:

\section{Definition 14.1:}

Das Auflösungsvermögen ist der kleinste Abstand zweier Objektpunkte, die von einem optischen Gerät noch getrennt abgebildet werden können.

Problematisch dabei ist, dass es kein objektives Kriterium dafür gibt, wann zwei Beugungsscheibchen noch getrennt wahrgenommen werden. Oft wird das Rayleighsche Kriterium benutzt: 


\section{Definition 14.2:}

Rayleighsches Kriterium: Zwei Objekte werden dann sicher aufgelöst, wenn das nullte Beugungsmaximum des ersten Objekts mit dem ersten Beugungsminimum des zweiten zusammenfällt. Für den Winkel $\delta$, unter dem diese beiden Objekte mit Öffnung $b$ bei der Wellenlänge $\lambda$ erscheinen, gilt dann $\sin \delta=1,22 \lambda / b$.

Das Auflösungsvermögen $A$ eines Mikroskops bestimmt sich aus dem kleinsten Abstand $x_{\min }$ zweier Gegenstandspunkte, welche im Mikroskop aufgelöst werden können. Hierfür gelten die gleichen Bedingungen wie beim Fernrohr nach AвbE und RAYLEIGH. Die entscheidenden Größen bilden die Wellenlänge $\lambda$ des verwendeten Beobachtungslichtes, sowie die Numerische Apertur. Das Produkt aus der Brechzahl $n$ des Mediums zwischen Gegenstand und Objektiv und dem Sinus des Winkels $\alpha$ zwischen optischer Achse und dem Randstrahl des Lichtkegels, welcher noch gerade von einem Gegenstandspunkt und das Objektiv einzutreten vermag, heißt Numerische Apertur $N$ :

$$
N=n \cdot \sin \alpha .
$$

Somit ergibt sich für das optische Auflösungsvermögen die Gleichung

$$
x_{\min }=\frac{\lambda}{N}=\frac{\lambda}{n \sin \alpha} \text {. }
$$

\subsection{Fragen}

1. Das Mikroskop:

a. Zeichnen Sie den Strahlengang eines herkömmlichen Mikroskops und erklären Sie anhand der Zeichnung die Begriffe »Lupe«, »Bezugssehweite«, »optische Tubuslänge«.

b. Die Gesamtvergrößerung eines Mikroskops $V_{M}$ bestimmt sich aus Abbildungsmaßstab und Lupenvergrößerung zu

$$
V_{M}=-\frac{t \cdot s_{0}}{f_{\mathrm{Obj}} \cdot f_{\mathrm{Ok}}}
$$

mit $t=$ Tubuslänge, $s_{0}=$ deutliche Bezugssehweite $(25 \mathrm{~cm}), f$ Brennweite von Okular und Objektiv. Leiten Sie diese Formel aus der Abbildungsgleichung her.

c. Was bedeutet das negative Vorzeichen in der Formel?

d. Prinzipiell ist ein Mikroskop eine Kombination aus einem Fernrohr und einer Lupe: Erklären Sie das bitte (Zeichnung!).

2. Numerische Apertur und Auflösungsvermögen

a. Leiten Sie die Formel

$$
x_{\min }=\frac{\lambda}{n \cdot \sin \alpha}
$$

aus dem Abbe-Kriterium und einer einfachen Gitterüberlegung her. 
b. Erklären Sie die Bedeutung der Brechzahl $n$ anhand so genannter »Trocken-« und »Immersionsobjektive«. Wie groß kann die Apertur also maximal werden?

c. Welches ist die bestimmende Größe in der Auflösungsformel und begrenzt die Auflösung optischer Mikroskope (mit Größenangabe für sichtbares Licht)? Wie kann trotzdem die Auflösung eines »Mikroskops« erhöht werden?

\subsection{Weiterführendes}

1. Skizzieren Sie bitte den Strahlengang für das astronomische Fernrohr und berechnen Sie die Winkelvergrößerung.

\subsection{Durchführung}

\subsubsection{Teil A:}

Hierzu verwende man den Aufbau mit dem Motic-Mikroskop.

1. Messung der Gesamtvergrößerung des Mikroskops für die Objektive mit 10-facher und 40-facher Vergrößerung und dem senkrechten Okular: Als Objekt dient in diesem Fall das Objektmikrometer, welches am Objekttisch eingespannt wird. Der Vergleichsmaßstab wird neben den Objekttisch gestellt, so dass beide Maßstäbe parallel zueinander liegen. Blickt man nun (mit entspannten Augen) mit einem Auge in das Okular und mit dem anderen Auge auf den Vergleichsmaßstab, so kommen beide Bilder zur Deckung. In 3 Messungen für jedes Objektiv wird somit die Bildgröße bestimmt. Bei jeder Messung bitte neu fokussieren.

2. Man verwende das geneigte Okular mit dem kurzen verschiebbaren Tubus mit Mattscheibe. Durch Verschieben der Mattscheibe fokussiere man das reelle Zwischenbild des Objektivs (Bitte zweckmäßigerweise den Raum verdunkeln.). Bitte nicht am Objektivrad drehen. Man vermesse mit dem Messschieber das Objektivbild zur zugehörigen Objektiv-OkularKombination. Zweckmäßigerweise führe man die Teile 1 und 2 für jedes Objektiv und jede Fokussierung zusammen durch. 3 Messungen, für jedes Objektiv (1 pro Fokussierung)

3. Man vermesse nun die Objektivvergrößerungen durch Messung der Zwischenbilder für zwei verschiedene Tubuslängen und die beiden Objektive wie oben (10x, 40x), nämlich:

a. mit dem kurzen Tubus, der vollständig in den geneigten Okularhalter gesteckt wird.

b. mit dem langen Tubus, der ebenfalls vollständig in den geneigten Okularhalter gesteckt wird.

Bei jeder Messung bitte neu fokussieren!

Jeweils 3 Messungen. Bitte die beiden Tubuslängen $t_{1}, t_{2}$ messen.

\subsubsection{Teil B:}

Hierzu verwende man den Aufbau auf der optischen Schiene bestehend aus Leuchte, Farbfilter, Glasmaßstab, Spalt und Tubus. 
1. Das Mikroskop wird auf den Glasmaßstab scharf eingestellt. Dann wird die Aperturblende (Spalt direkt vor dem Objektiv) so weit geschlossen, dass die Maßstabteilung gerade nicht mehr aufgelöst wird. Notieren Sie den Abstand von Blende und Glasmaßstab.

2. Zur Bestimmung der Apertur dieser Anordnung wird nun der Glasmaßstab entfernt und

a. der Abstand von Aperturblende und Gegenstand durch Verschieben des Spaltes bis zu dessen Scharfstellung bestimmt;

b. die Spaltbreite mit dem Mikrometertrieb des Mikroskops ausgemessen. Notieren Sie die Vergrößerung des Mikroskops.

3. In der folgenden Messung besteht das Mikroskop aus der Leuchte, dem Farbfilter, dem Tubus sowie dem Plexiglasstab. Es wird auf die polierte ebene Vorderseite des Plexiglasstabes scharf eingestellt. Dann wird das Okular durch eine Lochblende ersetzt, die, ganz eingesteckt, in der Ebene des Zwischenbildes liegt. Durch die Lochblende wird die auf der Rückseite des Plexiglases eingeritzte Skala beobachtet und so der Bündeldurchmesser in der Skalenebene bestimmt. Hierzu wird die Anzahl an erkennbaren Linien notiert. (Beobachtung am besten mit seitlich aufgestellter Tischlampe). Die Länge des Plexiglasstabes wird vermessen.

\subsection{Angaben}

Die Skala des Objektmikrometers muss der Aufschrift des Metallrahmens entnommen werden; die Skala des Plexiglasstabes beträgt $0,5 \mathrm{~mm}$. Der Brechungsindex der Luft wird mit $n \approx 1$, der des Plexiglasstabes mit $n \approx 1,49$ angenommen.

\subsection{Auswertung}

\subsubsection{Teil A:}

1. Zeichnen Sie den Strahlengang.

2. Bestimmen Sie aus Messung 1. die Gesamtvergrößerung, aus Messung 2. die Objektivvergrößerung für beide Objektive und berechnen Sie daraus jeweils die Okularvergrößerung.

3. Berechnen Sie aus 3. die Brennweite $f$ der Objektive mit Fehlerangabe. Verwenden Sie hierbei, dass für die Vergrößerung gilt:

$$
V=\frac{B}{G}=\frac{b}{g}
$$

mit der Bild- und Gegenstandsgröße $B$ und $G$, sowie der Bild- und Gegenstandsweite $b$ und g. Zusammen mit der Linsengleichung erhält man

$$
V_{\text {lang }}-V_{\text {kurz }}=\frac{\Delta t}{f}
$$

mit der Differenz der Tubuslängen $\Delta t$. 


\subsubsection{Teil B:}

1. Zeichnen Sie den Strahlengang zu 1. und 3. .

2. Geben Sie das durch die Maßstabeinteilung gegebene Auflösungsvermögen zu 1. an.

3. Berechnen Sie die Breite des Spaltes und damit den Öffnungswinkel aus Messung 2. Hiermit können Sie nun mit der Annahme $n_{\text {Luft }} \approx 1$ das Auflösungsvermögen bestimmen.

4. Vergleichen Sie die beiden Ergebnisse für das Auflösungsvermögen.

5. Die Apertur und das Auflösungsvermögen des Mikroskops ist aus 3. zu bestimmen. Berechnen Sie hierzu die Breite $d$ der sichtbaren Fläche und daraus zusammen mit der Länge $L$ den Winkel der äußersten noch sichtbaren Lichtstrahlen im Plexiglasstab. Leiten Sie die Formel

$$
\sin \alpha=\frac{d / 2}{\sqrt{d^{2} / 4+L^{2}}}
$$

her. Hiermit können Sie nun die numerische Apertur und folglich das Auflösungsvermögen berechnen. 


\section{Messung der Lichtgeschwindigkeit}

Nach den aus den Maxwell-Gleichungen (JAMEs Clerk Maxwell, 1831-1879) hergeleiteten Wellengleichungen für elektromagnetische Felder ist die Ausbreitungsgeschwindigkeit elektromagnetischer Wellen im Vakuum unabhängig von deren übrigen Eigenschaften wie der Frequenz. Historisch wurden verschiedenste Methoden verwendet, um die Lichtgeschwindigkeit mit immer besserer Präzision zu bestimmen. Dies führte schließlich zum Definitionswert 2,997 92458 . $10^{8} \mathrm{~m} / \mathrm{s}$, anhand dessen die Längeneinheit 1 Meter definiert ist. Der gegenwärtige Versuch verwendet eine der moderneren Methoden zur Bestimmung der Lichtgeschwindigkeit und misst die Abhängigkeit der Lichtgeschwindigkeit vom durchlaufenen Medium.

\subsection{Stichworte}

Elektromagnetische Wellen, Ausbreitung, Wellengleichung, Laufzeitmessung, Lichtgeschwindigkeit in Medien.

\subsection{Literatur}

Empfohlen: Dem 2 [12]: Kap. 7.1, 7.2, 7.7, 8.1; Gerthsen [45]: Kap. 8.4.3, 10.3.2., 10.3.5. Weitere Literatur: BS-3.

\subsection{Zubehör}

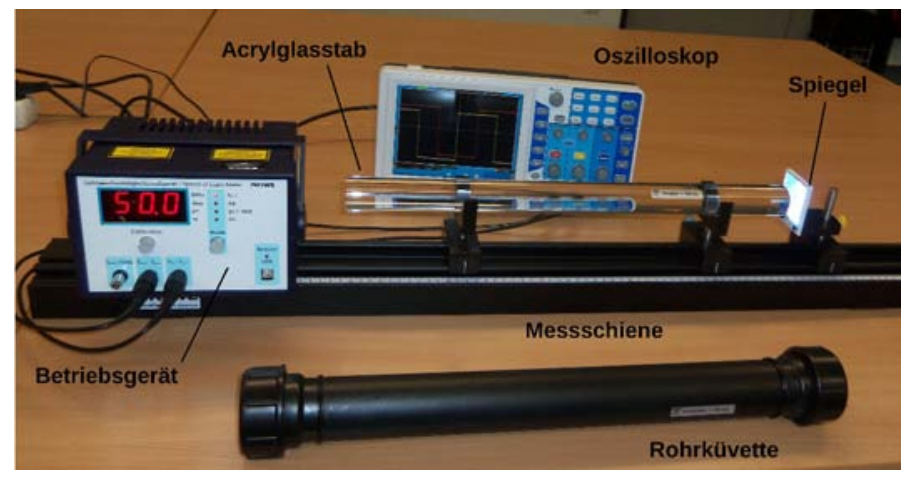

Bild 15.1: Der Versuch »Messung der Lichtgeschwindigkeit«.

Bild 15.1 zeigt ein Foto des Versuches mit Zubehör: Betriebsgerät mit Laser, Oszilloskop, Messschiene mit Reflexionsspiegel und Haltern für die zu untersuchenden Medien, Acrylglasstab, Rohrküvette mit Einlassscheiben (mit Wasser zu befüllen). 


\subsection{Grundlagen}

Nach dem Maxwell-Gleichungen gilt für das elektrische Feld $\vec{E}$ und das Magnetfeld $\vec{B}$ in einem ladungs- und stromfreien Medium mit Dielektrizitätskonstante $\varepsilon$ und Permeabilität $\mu$ :

$$
\begin{array}{lr}
\nabla \cdot \vec{E}=0, & \nabla \times \vec{E}+\frac{\partial \vec{B}}{\partial t}=0, \\
\nabla \cdot \vec{B}=0, & \nabla \times \vec{B}-\varepsilon \mu \frac{\partial \vec{E}}{\partial t}=0 .
\end{array}
$$

Unter Verwendung der Divergenzgleichungen führt die Kombination der beiden Rotationsgleichungen zu Wellengleichungen für die karthesischen Komponenten von $\vec{E}$ und $\vec{B}$, hier als $u$ bezeichnet:

$$
\nabla^{2} u-\varepsilon \mu \frac{\partial^{2} u}{\partial t^{2}}
$$

G1. (15.2) hat als Lösung eine ebene Welle mit der Ausbreitungsgeschwindigkeit $v$ im Medium

$$
v=\frac{1}{\sqrt{\varepsilon \mu}},
$$

so dass sich im Vakuum wegen $\varepsilon=\varepsilon_{0}$ ( $\varepsilon_{0}$ : elektrische Feldkonstante) und $\mu=\mu_{0}\left(\mu_{0}\right.$ : magnetische Feldkonstante) die Lichtgeschwindigkeit $c$ unabhängig von den Eigenschaften der Welle aus Naturkonstanten zu

$$
c=\frac{1}{\sqrt{\varepsilon_{0} \mu_{0}}}
$$

ergibt. Mit der Defnition des Brechungsindex $n$

$$
n:=\sqrt{\frac{\varepsilon \mu}{\varepsilon_{0} \mu_{0}}}
$$

ergibt sich dann die Lichtgeschwindigkeit in einem Medium zu

$$
v=\frac{c}{n} .
$$

Da im Allgemeinen Dielektrizitätskonstante und Permeabilität nicht nur material- sondern auch frequenzabhängig sind, ist damit die Lichtgeschwindigkeit in einem Medium $v$ abhängig von den Eigenschaften der elektromagnetischen Welle.

\subsection{Fragen}

1. Warum ist es möglich, die Vakuumlichtgeschwindigkeit als Definitionsgrundlage der Längeneinheit »Meter« zu verwenden? Warum kann hierfür die Ausbreitungsgeschwindigkeit anderer bekannter Wellen nicht herangezogen werden? 
2. Erläutern Sie den Ursprung der Material- und Frequenzabhängigkeit der Dielektrizitätskonstante und der Permeabilität.

\subsection{Durchführung}

Das Betriebsgerät sendet aus der Öffnung rechts einen gepulsten Laserstrahl aus, dessen Modulationssignal wie auch das empfangene Signal mit 1/1000-stel der Frequenz als Rechtecksignale an einem Oszilloskop dargestellt werden können. Schließen Sie die beiden Kanäle des Oszilloskops entsprechend an die beiden Ausgänge des Betriebsgeräts an. Das Betriebsgerät sollte vor Beginn der Messungen mindestens 15 min. im Betrieb aufgewärmt werden. Es empfiehlt sich, dies während der Vorbesprechung zu tun.

1. Justierung des Aufbaus: Positionieren Sie den Spiegel in einiger Distanz vom Betriebsgerät, entfernen Sie alle übrigen Teile aus dem Strahlengang. Stellen Sie das Betriebsgerät nun mit dem Mode-Knopf auf $\mathrm{f}_{\text {emit }}$-Betrieb. Spiegel und Betriebsgerät so gegeneinander justieren, dass über die gesamte Länge der Messschiene der Laserstrahl mittig auf den Spiegel fällt und in die Austrittsöffnung des Lasers reflektiert wird. Niemals direkt in den Laserstrahl blicken!

2. Kalibration des Aufbaus: Bringen Sie den Spiegel direkt vor die Austrittsöffnung des Lasers und notieren Sie dessen Position auf der Messschiene (dies ist der Nullpunkt der Messung). Stellen Sie das Betriebsgerät mit dem Mode-Knopf auf $\Delta \varphi$-Betrieb und drücken Sie den Calibration-Knopf. Auf dem Oszilloskop sollen nun zwei Rechteckkurven zu sehen sein (ggf. Einstellungen des Oszilloskops ändern), die zeitlich exakt überlappen.

3. Verschieben Sie nun den Spiegel auf Abstände größer als $50 \mathrm{~cm}$ bis zum Ende der Messschiene und messen Sie an 10 verschiedenen Positionen den Zeitunterschied zwischen den beiden Signalen auf dem Oszilloskop. Schätzen Sie dabei am Oszilloskop die Ablesegenauigkeit ab.

4. Deaktivieren Sie den Laser, indem Sie den Mode-Knopf so oft drücken, bis in der Anzeige »Off « erscheint. Bringen Sie nun den Acrylglasstab auf den beiden Haltern auf die Messschiene und positionieren Sie den Spiegel direkt an dessen rechtes Ende. Mit dem ModeKnopf auf $\Delta \varphi$-Betrieb gehen und den Calibration-Knopf drücken, so dass beide Signale wieder übereinander liegen. Die Position des Spiegels notieren. Entfernen Sie den Acrylglasstab, wodurch ein Laufzeitunterschied zwischen den Signalen entsteht. Den Spiegel so weit verschieben, bis beide Signale wieder überlappen und die Position des Spiegels notieren. Diese Prozedur mindestens fünf mal wiederholen. Messen Sie die Länge des Acrylglasstabs.

5. Führen Sie Messung 4 analog mit der mit Wasser gefüllten Rohrküvette durch.

\subsection{Angaben}

Der Laser ist mit einer Frequenz von $50 \mathrm{MHz}$ (max. 5\% Ungenauigkeit) gepulst.

Der Brechungsindex von Acrylglas beträgt $n_{\text {Acryl }}=1,49 \pm 0,01$, der von Wasser $n_{\text {Wasser }}=$ 1,3311 bei $\lambda_{\mathrm{C}}=656,3 \mathrm{~nm}[66]$. 


\subsection{Auswertung}

1. Tragen Sie den Wegunterschied aus Messung 3 (zusätzlichen Weg durch Reflexion beachten!) gegen den vom Oszilloskop abgelesenen Laufzeitunterschied auf (Faktor 1000 des Betriebsgeräts beachten!). Bestimmen Sie aus der Steigung die Lichtgeschwindigkeit in Luft.

2. Bestimmen Sie die Lichtgeschwindigkeit im jeweiligen Medium aus der gemessenen Positionsdifferenz des Spiegels in den Messungen 4 und 5 sowie aus der Länge des Acrylglasstabs bzw. des Wasservolumens in der Küvette. Berechnen Sie daraus den Brechungsindex der beiden Medien.

\subsection{Bemerkungen}

Sie arbeiten in diesem Versuch mit einem frei zugänglichen Laserstrahl. Beim Hantieren im Strahlbereich diesen immer deaktivieren (den Mode-Knopf so oft drücken, bis in der Anzeige »Off « erscheint), um unerwünschte Reflexionen z.B. an Schmuckstücken oder Armbanduhren zu vermeiden. Die Nulllage der Signale bleibt dabei erhalten, sofern sich der Laser nicht zu lange abkühlt. Den Laser nie ohne Spiegel im Strahlengang betreiben.

Nach Ende des Versuchs bitte die Rohrküvette entleeren, geöffnet zum trocknen liegen lassen und den Arbeitsbereich trocken hinterlassen. 


\section{Das Prismen- und Gitterspektrometer}

Mit Hilfe eines Prismas oder eines Gitters ist es möglich, das Licht in seine Spektralfarben aufzufächern. Über Dispersion und Interferenz sollen in diesem Versuch die Wellenlängen der Linien einer Hg-Cd-Lampe gemessen werden. Das Auflösungsvermögen des Prismen- und des Gitterspektrometers sollen bestimmt und miteinander verglichen werden.

\subsection{Stichworte}

Beugung, Brechung, Interferenz, kohärentes Licht, Huygens-Prinzip, Auflösungsvermögen, Rayleighsche Grenzlage, Dispersion, Fraunhofer-Formel

\subsection{Literatur}

NPP: 37, 38; BS-3: Optik; Gerthsen, Walcher: 4.0-4.7; Kohlrausch; Geschke; Dem-2; Optik, Hecht.

\subsection{Zubehör}

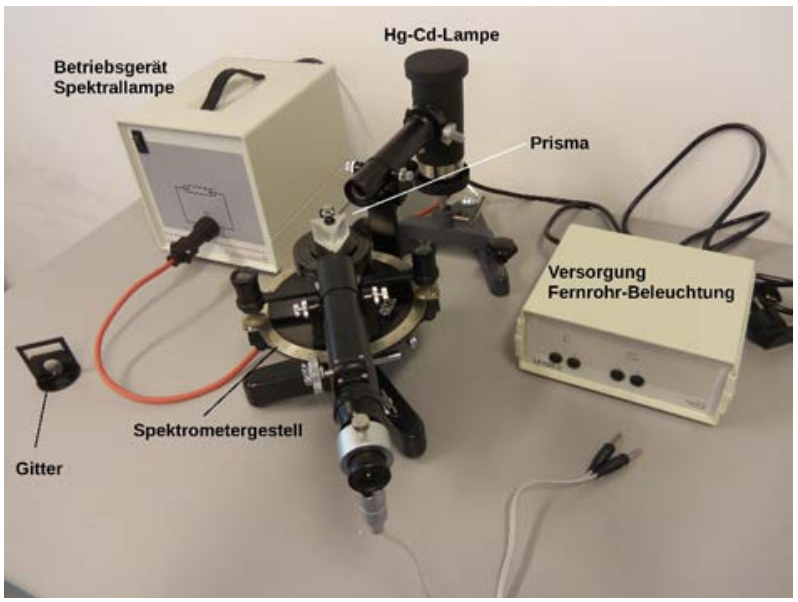

Bild 16.1: Der Versuch »Prismen- und Gitterspektrometer $\ll$.

Bild 16.1 zeigt ein Foto des Versuches mit Zubehör: Spektrometergestell mit Winkelskala, Fernrohr mit Betriebsgerät für Beleuchtung und Spaltrohr, Hg-Cd-Lampe mit Betriebsgerät, Prisma, Glasgitter. 


\subsection{Grundlagen}

\subsubsection{Das Prismenspektrometer}

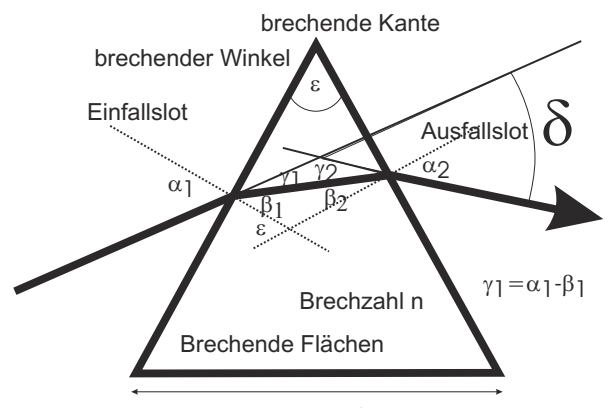

Basis S

Bild 16.2: Hauptschnitt eines Prismas.

Wird ein Lichtstrahl an der Grenzfläche zwischen zwei Medien gebrochen und weist eines der beiden Medien eine Dispersion auf, kommt es zu einer Zerlegung des Lichts in sein Spektrum. Weil beim Übergang von Luft auf Glas das blaue Licht stärker als das rote gebrochen wird und man anfangs nur diesen Fall kannte, nennt man diejenige Dispersion, bei der der Brechungsindex $n$ mit abnehmender Wellenlänge $\lambda$ zunimmt, normal $\left(\frac{d n}{d \lambda}<0\right)$, im umgekehrten Fall anomal $\left(\frac{d n}{d \lambda}>\right.$ $0)$.

Beim schiefen Durchgang durch ein von zwei planparallelen Grenzflächen eingeschlossenes homogenes Medium erfährt ein Lichtstrahl - und bei Dispersion auch jeder Anteil des Lichtstrahls mit unterschiedlicher Wellenlänge für sich - nur eine Parallelverschiebung, sofern Brechungsindex und Dispersion vor und hinter dem Medium gleich sind.

Ein optisches Prisma wird verkörpert durch ein homogenes prismatisch begrenztes Medium. Zwei der ebenen Begrenzungsflächen sind die brechenden Flächen. Sie schließen den brechenden Winkel $\epsilon$ ein und schneiden sich in der brechenden Kante. Dem brechenden Winkel $\epsilon$ gegenüber liegt die Basis als dritte Prismenfläche. Jede senkrecht zur brechenden Kante liegende Ebene ist ein Hauptschnitt des Prismas. Bei symmetrischem Strahlengang im Hauptschnitt ist die Gesamtablenkung $\delta$ des Lichtstrahls minimal. Für diesen Fall lässt sich mit Hilfe des SNELLIusschen Brechungsgesetz eine Beziehung zwischen dem minimalen Ablenkungswinkel und dem Brechungsindex $n$ ableiten, die Fraunhofer-Formel

$$
n=\frac{\sin \left(\left(\delta_{\min }+\varepsilon\right) / 2\right)}{\sin (\varepsilon / 2)} .
$$

Da die Lichtgeschwindigkeit im Medium $c$ und damit die Brechzahl $n$ von der Wellenlänge $\lambda$ abhängt, ist der Ablenkungswinkel $\delta$ für jede Wellenlänge verschieden: Beim Durchgang eines »weißen« Lichtbündels tritt eine spektrale Zerlegung auf. Die Größe

$$
\frac{d \delta}{d \lambda}=\left(\frac{\partial \delta}{\partial n}\right) \cdot \frac{d n}{d \lambda}
$$


nennt man Winkeldispersion des Prismas. Der erste Faktor errechnet sich aus der Fraunhoferschen Formel, der zweite Faktor $\frac{d n}{d \lambda}$ ist die Dispersion. Genaue Zahlenwerte für den Brechungsindex $n(\lambda)$ und somit der Dispersion $\frac{d n}{d \lambda}$ der im Praktikum verwendeten Prismenmaterialien finden Sie in Abschnitt 16.7 Möchte man das Prismenspektrometer für Wellenlängenmessungen einsetzten, so muss beachtet werden, dass auch ein monochromatischer, infinitesimal schmaler Eingangsstrahl sich nach der Ablenkung durch das Prisma als Beugungsmuster zeigt. Man hat es hier also nicht nur mit einer einfachen geometrischen Abbildung zu tun. Wie gut ein Prismenspektrometer zur Messung zweier dicht benachbarter Wellenlängen geeignet ist, wird über das Auflösungsvermögen

$$
A:=\frac{\lambda}{\Delta \lambda}
$$

angegeben. D. h. Licht mit der Wellenlänge $\lambda$ ist von Licht der Wellenlänge $\lambda+\Delta \lambda$ genau dann gerade noch aufzulösen, wenn das $k$. Hauptmaximum des Beugungsmusters der Wellenlänge $\lambda$ genau auf das 1. Minimum neben dem $k$. Hauptmaximum des Beugungsmusters der Wellenlänge $\lambda+\Delta \lambda$ fällt (Rayleighsche Grenzlage). Für das Prisma lässt sich Gl. (16.3) damit durch

$$
A=B\left|\frac{d n}{d \lambda}\right|
$$

ausdrücken. $B$ ist die effektive Basisbreite, über deren Variation durch die Spaltbreite $S$ im Versuch das Auflösungsvermögen $A$ bestimmt wird.

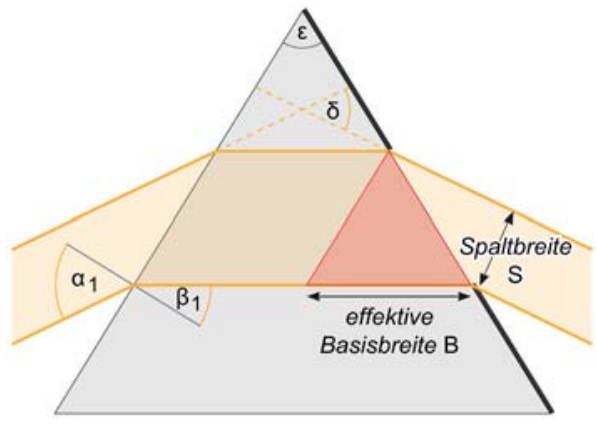

Bild 16.3: Skizze zur Bestimmung des Auflösungsvermögens eines Prismas über Spaltbreite $S$ und die effektive Basisbreite $B$.

\subsubsection{Das Gitterspektrometer}

Da die Funktionsweise des Gitterspektrometers auf Interferenzerscheinungen beruht, wird im Versuch, wie schon beim Prisma, mit einer Spalt / Linsenkombination kohärentes Licht hergestellt. Beim Durchgang durch das Gitter entsteht an jeder (vereinfacht: infinitesimal kleinen) Gitteröffnung eine Huygenssche Elementarwelle. Diese Wellen interferieren miteinander, so dass man durch das Messokular ein Interferenzstreifenmuster erkennen kann. Da dieses Muster von der Wellenlänge abhängt, kann auch ein Gitter als Spektralapparat eingesetzt werden. 
Auch das Auflösungsvermögen des Gitters ist über die Rayleighsche Grenzlage (Gl. [16.3) gegeben. Zusammen mit den Gleichungen für die Richtungen $\alpha$ der Hauptmaxima und Minima für die Interferenz am Gitter ( $k$ : Ordnung, $N$ : Anzahl der beleuchteten Spalte, $a$ : Gitterkonstante)

$$
\begin{aligned}
& \sin \alpha_{\text {max }}=\frac{k \lambda}{a} \\
& \sin \alpha_{\text {min }}=\frac{n \lambda}{N a}
\end{aligned}
$$

lässt sich das Auflösungsvermögen durch

$$
A=k N
$$

ausdrücken. $n$ ist dabei eine ganze Zahl zwischen 1 und (N-1), zwischen $(\mathrm{N}+1)$ und (2N-1), usw., wobei $n=0, N, 2 N, \ldots$ ausgeschlossen sind. Zwischen den Hauptmaxima liegen also eine von $N$ abhängige Zahl von Nebenminima. Im Praktikumsversuch wird das Auflösungsvermögen $A$ durch Variation der Zahl der beleuchteten Spalte $N$ bestimmt.
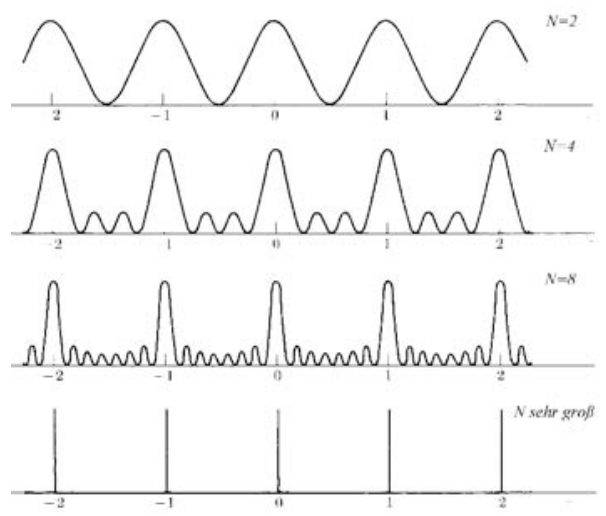

Bild 16.4: Interferenzmuster eines Gitters mit infinitesimal kleinen Gitteröffnungen. $N$ : Anzahl der beleuchteten Spalte. Die Gitterkonstante $a$ ist konstant.

\subsection{Fragen}

1. Wie stellt man paralleles kohärentes Licht her?

2. Wie leitet man für das Prisma Gleichung (16.1) und damit Gl. 16.4) ab?

3. Wie leitet man für das Gitter die Gleichungen (16.5), 16.6) und damit Gl. (16.7) ab?

4. Warum muss beim Prisma mit der effektiven Basislänge gerechnet werden?

5. Warum verwendet man zur Spektroskopie nicht grundsätzlich nur Gitter? Durch Beobachtung einer hohen Ordnung $k$ lässt sich das Auflösungsvermögen doch beliebig erhöhen? 


\subsection{Durchführung}

\subsubsection{Das Prismenspektrometer}

1. Justierung des Spektralapparates.

- Spalt von außen mit der Spektrallampe beleuchten. Dabei beachten, dass sich die Lampe in der optischen Achse des Spaltrohres befindet.Fernrohr auf das Spaltrohr richten und den Spalt mit der Mikrometerschraube zur Spaltverbreiterung etwas öffnen. Spalt mit Schieber auf eine beobachtbare, geeignete Spalthöhe einstellen. Mit der Höhenverstellschraube des Spaltrohres den Spalt mittig zum Horizontalfaden des Fadenkreuzes ausrichten und anschließend kontern. Feststellschraube für Spaltauszug lösen und Spalttubus entlang der Achse so verschieben, dass er scharf abgebildet wird. Durch Drehen des Tubus den Spalt vertikal ausrichten, so dass er parallel zum Vertikalfaden des Fadenkreuzes ist, und Feststellschraube für Spaltauszug wieder anziehen.

- Fernrohr in einem spitzen Winkel zum Spaltrohr drehen und mit Feststellschraube fixieren. Prisma auf den Halter des Prismentisches stellen, so dass eine Prismenfläche parallel zur Verbindungslinie zweier Nivellierschrauben des Prismentisches verläuft. Prismentisch drehen, bis das von einer Prismenfläche reflektierte Spaltbild im Fernrohr sichtbar wird, und mit Feststellschraube für den Prismentisch fixieren. Mit hinterer Nivellierschraube des Prismentisches den reflektierten Spalt in die Mitte des Fadenkreuzes bringen.

- Die beiden folgenden Schritte so oft wiederholen, bis sich die Reflexion des Spaltes beim Drehen des Prismentisches nicht mehr vertikal verschiebt:

- Feststellschraube des Prismentisches lösen, Prismentisch drehen, bis das Spaltbild an der anderen Prismenfläche reflektiert wird, und mit Feststellschraube für Prismentisch wieder fixieren.

- Mit der jetzt aus der Sicht des Fernrohrs hinteren Nivellierschraube des Prismentisches den reflektierten Spalt in die Mitte des Fadenkreuzes bringen.

2. Prisma nun so in den Strahl bringen, dass es durchleuchtet wird. Stellen Sie durch Drehen des Prismas auf dem Teller den minimalen Ablenkungswinkel her und justieren Sie den Schwenkarm auf eine der gelben Linien $\left(\lambda_{\text {gelb }}\right)$. Dieser Winkel ist zu notieren.

3. Bestimmen Sie nun den Unterschied $\Delta \varphi$ des Ablenkungswinkels der gelben Linie aus Messung $2\left(\lambda_{\text {gelb }}\right)$ zur grünen Linie $\left(\lambda_{\text {grün }}\right)$ mit dem Feintrieb. Der Grobtrieb des Schwenkarms muss dafür arretiert werden!

4. Verengen Sie den Bündelquerschnitt $S$ des auf das Prisma fallenden Strahls durch den Spalt so lange, bis die beiden gelben Linien gerade noch getrennt erscheinen. Notieren Sie die Einstellung des Mikrometertriebs am Spalt bei dieser Einstellung und bei völlig geschlossenem Spalt.

Messen Sie die geometrische Basislänge des Prismas. 


\subsubsection{Das Gitterspektrometer}

1. Mit Hg-Cd-Lampe und Fern-/Spaltrohr ist wie im ersten Versuchsteil ein scharfes Bild des Spaltes ohne Objekt auf dem Tisch herzustellen. Nun wird das Gitter in den Strahlengang gebracht. Bitte achten Sie darauf, dass das einfallende parallele Licht möglichst senkrecht auf das Glasgitter trifft.

2. Bestimmen Sie die Ablenkungswinkel der gelben, grünen und violetten (Doppel)Linie für die 1. und 2. Ordnung.

3. Bestimmen Sie die Winkeldifferenz $\Delta \alpha$ zwischen den beiden gelben Linien und die Winkeldifferenz $\Delta \beta$ zwischen den grünen und einer der gelben Linien (ebenfalls für die 1. und 2. Ordnung).

4. Mit dem Mikrometertrieb des Spalts bestimme man $S$, für die die beiden gelben Linien gerade nicht mehr getrennt sichtbar sind (ebenfalls für 1., 2. Ordnung).

Vorsicht: Das Glasgitter ist sehr empfindlich. Daher darf das Gitter nicht berührt werden!

\subsection{Angaben}

Tabelle 16.1: Wellenlängen der Emissionslinien von Quecksilber (Hg) und Cadmium (Cd) (nach [66]).

\begin{tabular}{lll}
\hline Wellenlänge & Farbe & Intensität \\
\hline$\lambda_{1}=708,19 \mathrm{~nm}(\mathrm{Hg})$ & rot & schwach \\
$\lambda_{2}=690,72 \mathrm{~nm}(\mathrm{Hg})$ & rot & schwach \\
$\lambda_{3}=643,85 \mathrm{~nm}(\mathrm{Cd})$ & rot & stark \\
$\lambda_{4}=635,99 \mathrm{~nm}(\mathrm{Cd})$ & gelbrot & schwach \\
$\lambda_{5}=579,07 \mathrm{~nm}(\mathrm{Hg})$ & gelb & sehr stark \\
$\lambda_{6}=576,96 \mathrm{~nm}(\mathrm{Hg})$ & gelb & sehr stark \\
$\lambda_{7}=546,07 \mathrm{~nm}(\mathrm{Hg})$ & grün & stark \\
$\lambda_{8}=508,58 \mathrm{~nm}(\mathrm{Cd})$ & grün & stark \\
$\lambda_{9}=491,60 \mathrm{~nm}(\mathrm{Hg})$ & blaugrün & mittel \\
$\lambda_{10}=479,99 \mathrm{~nm}(\mathrm{Cd})$ & blaugrün & stark \\
$\lambda_{11}=467,82 \mathrm{~nm}(\mathrm{Cd})$ & blau & stark \\
$\lambda_{12}=467,82 \mathrm{~nm}(\mathrm{Cd})$ & blau & stark \\
$\lambda_{13}=441,46 \mathrm{~nm}(\mathrm{Cd})$ & blau & mittel \\
$\lambda_{14}=407,78 \mathrm{~nm} \mathrm{(Hg)}$ & violett & mittel \\
$\lambda_{15}=404,66 \mathrm{~nm}(\mathrm{Hg})$ & violett & mittel \\
\hline
\end{tabular}

Das hier verwendete Flintglas-Prisma hat nach Herstellerangaben einen Brechungsindex von $n=1,620$ bei $\lambda_{\mathrm{D}}=589,3 \mathrm{~nm}$. und eine mittlere Dispersion von $\Delta n=0,017$ zwischen den NatriumD- und Wasserstoff-F-Linien $\left(\lambda_{\mathrm{D}}=589,3 \mathrm{~nm}, \lambda_{\mathrm{F}}=486,1 \mathrm{~nm}\right)$.

Die Gitterkonstante des Glasgitters beträgt $a=5700 \mathrm{~cm}^{-1}$.

Die Wellenlängen einiger Hg-Cd-Emissionslinien sind in Tabelle 16.1 angegeben. 


\subsection{Auswertung}

\subsubsection{Das Prismenspektrometer}

1. Zeichnen Sie den Strahlengang des Versuchs.

2. Aus allen mit der Winkelskala gemessenen Größen sind unter Berücksichtigung der Nullposition die tatsächlichen Winkeldifferenzen zu bestimmen.

3. Bestimmen Sie aus Messung 3 und aus den bekannten Wellenlängen der gemessenen Linien die Dispersion $d n / d \lambda$ der beiden gelben Linien (die Dispersion wird als konstant angenommen). Vergleichen Sie die berechnete Dispersion mit dem angegebenen Wert für Flintglas.

4. Vergleichen Sie für die gemessene Spaltbreite $S$ das Auflösungsvermögen nach G1. 16.4 mit dem Auflösungsvermögen nach Gl. 16.3.

5. Berechnen Sie das mit dem Prisma maximal erreichbare Auflösungsvermögen (Annahme: die gesamte geometrische Basislänge wird ausgeleuchtet). Welcher kleinsten, noch trennbaren Wellenlängendifferenz entspricht das?

\subsubsection{Das Gitterspektrometer}

1. Aus den gemessenen Ablenkungswinkeln und aus $\Delta \alpha$ sowie $\Delta \beta$ berechne man die Gitterkonstante des Glasgitters und die Wellenlängendifferenz $\Delta \lambda$ der gelben Doppellinien.

2. Berechnen Sie aus den für die verschiedenen Ordnungen erhaltenen Werten für $S$ das Auflösungsvermögen nach G1. 16.7 und vergleichen Sie es mit dem Auflösungsvermögen nach Gl. 16.3).

3. Geben Sie das maximal erreichbare Auflösungsvermögen in der 1. Ordnung an.

4. Berechnen Sie die Wellenlänge der violetten Linie der Hg-Lampe und Vergleichen Sie sie mit dem Literaturwert.

Vergleichen Sie die beiden Spektrometer. 


\section{Fresnelsche Formeln und Polarisation}

Dieser Versuch führt in die Theorie der Polarisation von Licht und die Abhängigkeiten von Reflexion und Transmission vom (komplexen) Brechungsindex und dem Einfallswinkel ein.

\subsection{Stichworte}

Fresnelsche Formeln, Brewster-Winkel, Reflexion, (komplexer) Brechungsindex, Polarisation, Polarisator, Analysator, Polarisationsfilter.

\subsection{Literatur}

BS-3: Optik, S.455-535; ; Wap: 211ff, Gerthsen: 508ff; NPP: 41-43; Dem-2; Geschke.

\subsection{Zubehör}

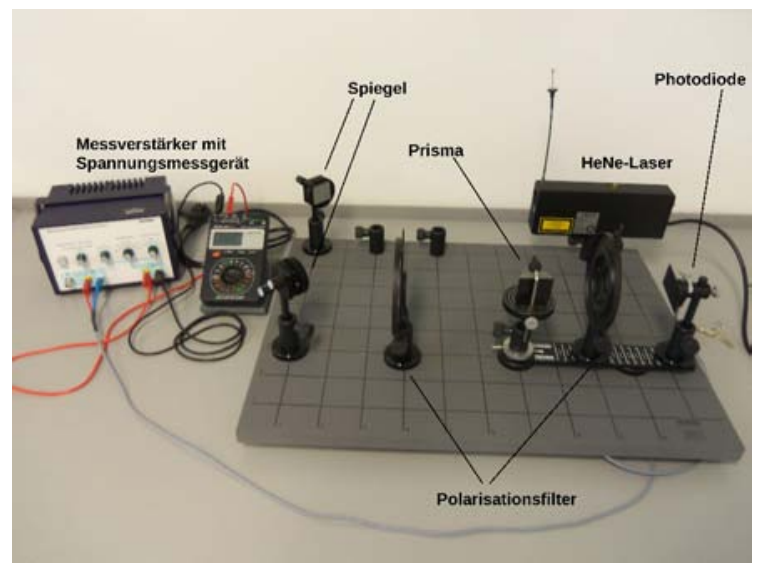

Bild 17.1: Der Versuch »Fresnelsche Formeln und Polarisation«.

Bild 17.1 zeigt ein Foto des Versuchsaufbaus mit Zubehör: HeNe-Laser, Optische Platte mit Haltern, 2 Spiegel, 2 Polarisationsfilter, Schwenkarm mit Prismenteller und Fotodiode, 1 Glasprisma, Messverstärker für die Fotodiode mit Multimeter.

\subsection{Grundlagen}

Aus den Maxwell-Gleichungen folgt direkt, dass elektromagnetische Wellen Transversalwellen sind. Experimentell war dieses Phänomen jedoch viel eher durch die Tatsache bekannt, dass sich elektromagnetische Strahlung polarisieren lässt. 
Thema des Versuches ist die unterschiedliche Erzeugung von polarisiertem Licht. Eine Methode verwendet Polarisationsfolien. Diese bestehen aus ausgerichteten dichroitischen Kristallen, mit denen sowohl polarisiertes Licht erzeugt (Polarisator), als auch die Polarisationsrichtung von Licht gemessen werden kann (Analysator). Die Kombination aus beiden wird im Versuch verwendet, um ein Polarimeter aufzubauen und eine weitere Polarisationsmethode zu untersuchen: die Polarisation infolge von Reflexion an einem dielektrischen Medium, im Falle des Versuches an einem Glasprisma. Letzteres wird durch die so genannten Fresnelschen Formeln (Augustin-Jean Fresnel, 1788- 1827) beschrieben, welche das Reflexions- und Transmissionsverhältnis elektromagnetischer Strahlung an einer Grenzfläche bestimmen. Da diese Formeln für die jeweilige Polarisationsrichtung des Lichtes relativ zur Grenzfläche verschieden sind, wird die Schwingungsebene des Lichtes bei Reflexion gedreht. Diese Drehung kann mit dem Analysator beobachtet und hierüber die Gültigkeit der Fresnelschen Formeln verifiziert werden. Die parallele Polarisation verschwindet bei Einfall im so genannten Brewster-Winkel. Dieser ist abhängig vom Brechungsindex des Glasprismas und soll somit im Versuch bestimmt werden.

\subsubsection{Theorie}

\section{Definition 17.1:}

Elektromagnetische Wellen und Polarisation: Elektromagnetische Wellen sind immer Transversalwellen. Die Polarisation wird nach der Schwingungsrichtung des elektrischen Feldvektors definiert, sie heißt Polarisationsrichtung.

Die hier verwendeten flachen Polarisatoren arbeiten mit Hilfe des Dichroismus, bei dem optische Anisotropie dafür sorgt, dass der Absorptionskoeffizient bei einer bestimmten Wellenlänge von der Polarisationsrichtung des einfallenden Lichts abhängt.
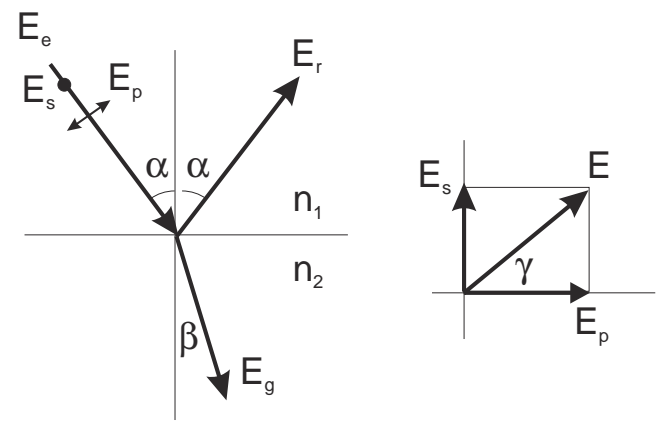

Die Basis für diesen Versuch bilden die Fresnelschen Formeln. Trifft Licht auf eine optische Grenzfläche, so gilt das Snelliussche Brechungsgesetz:

$$
n_{1} \sin \alpha=n_{2} \sin \beta
$$

Haben wir eben die Richtungen bestimmt, so bestimmen die Fresnelschen Formeln das Intensitätsverhältnis zwischen reflektiertem und gebrochenem Anteil des Lichtstrahls. 
Die Intensität einer Lichtwelle ist proportional zu $\sqrt{\epsilon} E^{2}$ (warum?). $E$ ist der Betrag des elektrischen Feldvektors. Die Intensität senkrecht zur Grenzfläche ist eine stetige Funktion, also gilt an der Grenzfläche:

$$
\sqrt{\epsilon_{1}}\left(E_{e}^{2}-E_{r}^{2}\right) \cos \alpha=\sqrt{\epsilon_{2}} E_{g}^{2} \cos \beta,
$$

wobei $E_{e}$ den einfallenden, $E_{r}$ den reflektierten und $E_{g}$ den gebrochenen Lichtstrahl beschreibt.

Die Parallelkomponente des elektrischen Feldes ist stetig an der Grenzfläche (warum?), also gilt für Polarisation senkrecht zur Einfallsebene:

$$
E_{e}+E_{r}=E_{g}
$$

Mit der Maxwell-Relation:

$$
\sqrt{\frac{\epsilon_{2}}{\epsilon_{1}}}=\frac{n_{2}}{n_{1}}=\frac{\sin \alpha}{\sin \beta}
$$

folgt:

$$
\begin{aligned}
& E_{r}=-E_{e} \frac{\sin (\alpha-\beta)}{\sin (\alpha+\beta)} \\
& E_{g}=E_{e} \frac{2 \sin \beta \cos \alpha}{\sin (\alpha+\beta)}
\end{aligned}
$$

Ist die Welle parallel zur Einfallsebene polarisiert, so ist nur die Komponente parallel zur Grenzfläche stetig:

$$
\left(E_{e}+E_{r}\right) \cos \alpha=E_{g} \cos \beta
$$

Es folgt:

$$
\begin{aligned}
& E_{r}=-E_{e} \frac{\tan (\alpha-\beta)}{\tan (\alpha+\beta)} \\
& E_{g}=E_{e} \frac{2 \sin \beta \cos \alpha}{\sin (\alpha+\beta) \cos (\alpha-\beta)}
\end{aligned}
$$

Mit der Definition $\varrho=E_{r} / E_{e}$ (Reflexionsverhältnis) und $\sigma=E_{g} / E_{e}$ (Durchlässigkeit) erhält man die Fresnelschen Formeln (A.-J. Fresnel, 1822):

E senkrecht zur Einfallsebene:

$$
\begin{gathered}
\varrho=-\frac{\sin (\alpha-\beta)}{\sin (\alpha+\beta)} \\
\sigma=\frac{2 \sin \beta \cos \alpha}{\sin (\alpha+\beta)}
\end{gathered}
$$


E parallel zur Einfallsebene:

$$
\begin{aligned}
\varrho & =-\frac{\tan (\alpha-\beta)}{\tan (\alpha+\beta)} \\
\sigma & =\frac{2 \sin \beta \cos \alpha}{\sin (\alpha+\beta) \cos (\alpha-\beta)}
\end{aligned}
$$

Mit dem Betrag des Reflexionsverhältnisses $|\varrho|$ kann man das in Bild 17.3 dargestellte Diagramm erstellen.

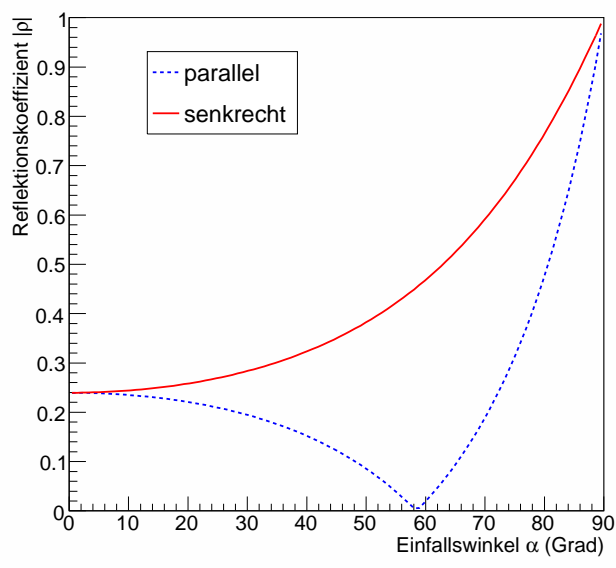

\subsubsection{Versuchsaufbau}

Das Polarimeter, in Bild 17.4 schematisch dargestellt, besteht aus zwei hintereinander angeordneten Polarisationsfiltern, dem Polarisator $\left(\mathrm{P}_{1}\right)$ und dem Analysator $\left(\mathrm{P}_{2}\right)$, der an den Schwenkarm für den zweiten Versuchsteil angebracht wird. Die Lichtquelle ist ein Helium-Neon-Laser $(\lambda=632,8 \mathrm{~nm})$. Zwischen Polarisator und Analysator trifft das Lichtbündel auf das Untersuchungsmedium, in diesem Fall ein Glasprisma. Da sich der Prismenteller nicht mit dem Schwenkarm mitdreht, muss während der Durchführung das Prisma nachjustiert werden, so dass der reflektierte Laserstrahl immer auf die Fotodiode fällt. Die Fotodiode wird über einen Verstärker und ein daran angeschlossenes Multimeter ausgelesen. Der Verstärker muss so eingestellt sein, dass die maximale zu untersuchende Lichtintensität ein Ausgangssignal am Multimeter unter $10 \mathrm{~V}$ erzeugt.

\subsection{Fragen}

1. Erklären Sie bitte anhand der Maxwellschen Gleichungen, dass elektromagnetische Wellen Transversalwellen sind.

2. Erklären sie die Begriffe lineare, elliptische und zirkulare Polarisation. 


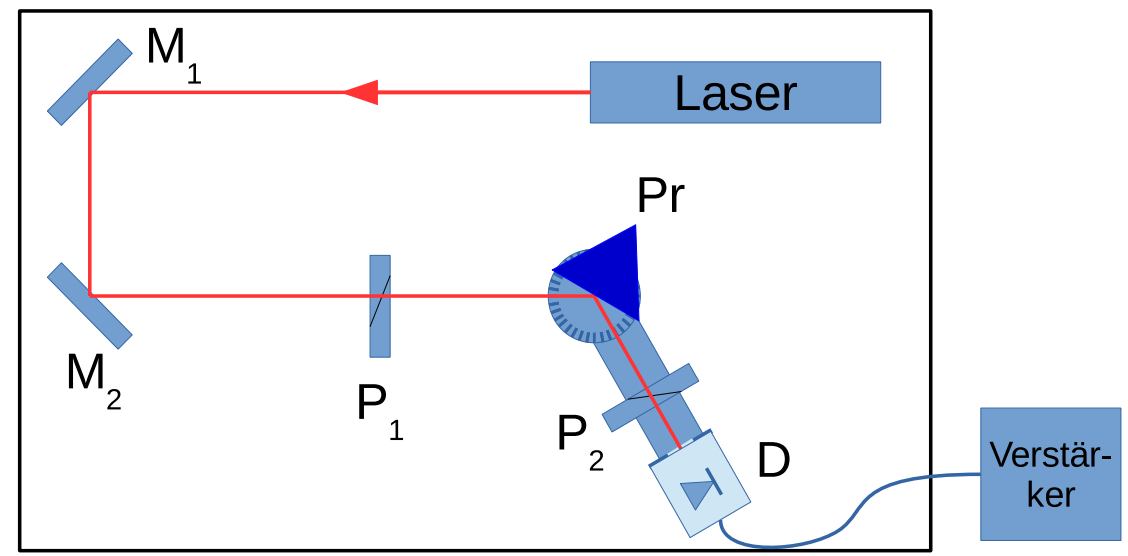

Bild 17.4: Skizze des Polarimeters mit Bestandteilen: Laser: HeNe-Laser, $\mathrm{M}_{1,2}$ : Spiegel,

$\mathrm{P}_{1}$ :Polarisationsfilter, $\mathrm{P}_{2}$ : Analysatorfilter, Pr: Glasprisma, D: Fotodiode.

3. Wie lässt sich das Snelliussche Brechungsgesetz geometrisch herleiten?

4. Welche Eigenschaften müssen optisch anisotrope Kristalle besitzen?

5. Was passiert bei $n_{2}>n_{1}(\alpha>\beta)$ mit den Vorzeichen von $E_{e}$ und $E_{g}$ ? Was bedeutet das physikalisch?

6. Erläutern Sie anhand der Fresnelschen Formeln die Phänomene im Brewster-Winkel ( $n=$ $\tan \alpha$ ) und die Totalreflexion. Tragen Sie hierzu das Reflexionsverhältnis und die Durchlässigkeit (Transmittivität) als Funktion des Einfallswinkels sowohl für $n_{2}>n_{1}$ als auch für $n_{2}<n_{1}$ auf.

\subsection{Durchführung}

Der Laser und der Messverstärker sollten vor Beginn des Versuchs mindestens 15 Minuten aufwärmen. Es empfiehlt sich, dies während der Vorbesprechung zu tun. Der Laser wird mit dem beiliegenden Schlüssel am Lasergehäuse selbst angeschaltet. An den Ausgang des Verstärkers wird ein Multimeter zur Spannungsmessung angeschlossen. Der Verstärker sollte auf folgende Parameter eingestellt werden:

- «low drift»: $R_{e}=10^{4} \Omega$

- amplification $10^{2}, V_{\max } \leq 10 \mathrm{~V}$.

- «Time Constant» 0

- Mittels Knopf, der mit einem Pfeil-Symbol gekennzeichnet ist, die Nulllage des Messverstärkers justieren.

1. Für den ersten Versuchsteil wird der Analysator $P_{2}$ aus dem Strahlengang entfernt und der Zeiger des Polarisators $\mathrm{P}_{1}$ auf 90 gestellt, so dass das Licht parallel zur Einfallsebene polarisiert wird. 
2. Entfernen Sie das Prisma vom Teller und stellen Sie den Schwenkarm gerade, so dass das Laserlicht direkt auf die Fotodiode fällt: Einstellen der Verstärkung, so dass die Spannung am Multimeter maximal $10 \mathrm{~V}$ beträgt. Dies ist die Referenzmessung der einfallenden Lichtintensität.

3. Das Prisma wird wieder auf dem Teller abgestellt. Stellen Sie den Schwenkarm auf einen Winkel $\phi=10^{\circ}$ ein und richten das Prisma so aus, dass der reflektierte Laserstrahl die Fotodiode trifft. Nach dem Snelliussche Brechungsgesetz sollte somit für den Einfallswinkel $\alpha=\phi / 2$ gelten. Die von der Fotodiode gemessene Lichtintensität wird notiert.

4. Wiederholen Sie dies für Werte von $\phi$ bis $160^{\circ}$ in Schritten von $5^{\circ}$ (in der Nähe des BrewsterWinkels in Schritten von $2.5^{\circ}$ ).

5. Punkte 2 bis 4 werden wiederholt, ebenfalls ohne den Analysator $\mathrm{P}_{2}$, aber mit dem Zeiger des Polarisators $\mathrm{P}_{1}$ auf 0 , so dass das Licht nun senkrecht zur Einfallsebene polarisiert ist.

6. Der Analysator $\mathrm{P}_{2}$ wird nun auf dem Schwenkarm zwischen Prisma und Fotodiode angebracht. Der Polarisator $\mathrm{P}_{1}$ wird auf einen Winkel von $45^{\circ}$ gestellt (Zeiger auf 45). Für die selben Werte von $\phi$ wie oben wird der Winkel des Analysators bestimmt, bei dem die minimale Intensität des reflektierten Lichts gemessen wird, d.h. der Winkel, bei dem die Polarisationsebene des Analysators senkrecht auf der des reflektierten Lichts steht.

\subsection{Angaben}

Der Brechungsindex des Glasprismas (Flintglas) beträgt im untersuchten Wellenlängenbereich $n=1,63$.

\subsection{Auswertung}

1. Tragen Sie die auf die einfallende Intensität normierte Intensität des reflektierten Lichts für senkrecht und parallel polarisiertes Licht auf und vergleichen Sie dies mit der Erwartung nach Gl. (17.10) und 17.12.

2. Bestimmen Sie aus der Messung den Brewster-Winkel und daraus den Brechungsindex des Glasprismas.

3. Bestimmen Sie die Drehung $\gamma$ der Schwingungsebene des Lichtes und tragen Sie diese grafisch über dem Einfallswinkel $\alpha$ auf.

4. Bestimmen Sie für den gemessenen Einfallswinkel die Drehung der Schwingungsrichtung des Lichtbündels nach folgender Formel:

$$
\tan \left(\gamma+\frac{\pi}{4}\right)=-\frac{\cos (\alpha-\arcsin (\sin \alpha / n))}{\cos (\alpha+\arcsin (\sin \alpha / n))}
$$

Tragen Sie die Werte bitte in die Grafik mit ein und vergleichen Sie Theorie und Experiment. 
5. Bestimmen Sie grafisch aus dem Drehwinkel von $45^{\circ}$ den Brechungsindex des Glasprismas. Warum geht das? Rechnen sie dabei eine Ableseungenauigkeit von $\pm 0.1^{\circ}$ mit ein. Vergleichen Sie diesen Wert des Brechungsindex mit dem Resultat von Punkt_2.

\subsection{Bemerkungen}

Sie arbeiten in diesem Versuch mit einem frei zugänglichen Laserstrahl. Beim Hantieren im Strahlbereich Schmuckstücke, Armbanduhren oder dergleichen ablegen, um unerwünschte Reflexionen zu vermeiden und nicht in Augenhöhe zur Strahlebene (d.h. im Sitzen) arbeiten. Den Laser nie ohne die Stellwände zwischen den Aufbauten betreiben. 


\section{Beugung und Interferenz von Laserlicht}

Dieser Versuch führt ein in Theorie, Erzeugung und Eigenschaften von Laserstrahlung und verwendet sie, um Beugungs- und Interferenzeffekte zu beobachten (Spalt, Loch, Gitter, Steg, Doppelloch). Der Intensitätsverlauf der jeweiligen Beugungsfigur wird elektronisch mittels Fotodiode, Schrittmotor und Computer aufgezeichnet.

\subsection{Stichworte}

Laser: Quantisiertes Atommodell, Metastabile Zustände, optische Übergänge, Strahlungsdichte im thermischen Gleichgewicht, Besetzungsinversion, stehende Wellen und Resonatorbedingung. Beugung und Interferenz: Beugung im Grenzfall der ebenen Welle, Wellen-Amplitude und -Intensität, Intensität komplementärer Gegenstände.

\subsection{Literatur}

Laser BS-3; NPP; Wap; Gerthsen; Geschke; Dem-2; H. Haken, H.C. Wolf: »Atom- und Quantenphysik« [27]; T. Mayer-Kuckuk: »Atomphysik« [43]; H. Weber, G. Herziger: »Laser: Grundlagen und Anwendungen« [67]; A. Cornay: »Atomic and Laser Spectroscopy« [7]; H. G. Kuhn: »Atomic Spectra« [36]; Kneubühl/Sigrist: Laser [32].

Beugung und Interferenz BS-3: Optik; Wap; NPP: 38-43; Gerthsen; Dem-2; Geschke; Lipson: Optik [39].

\subsection{Zubehör}

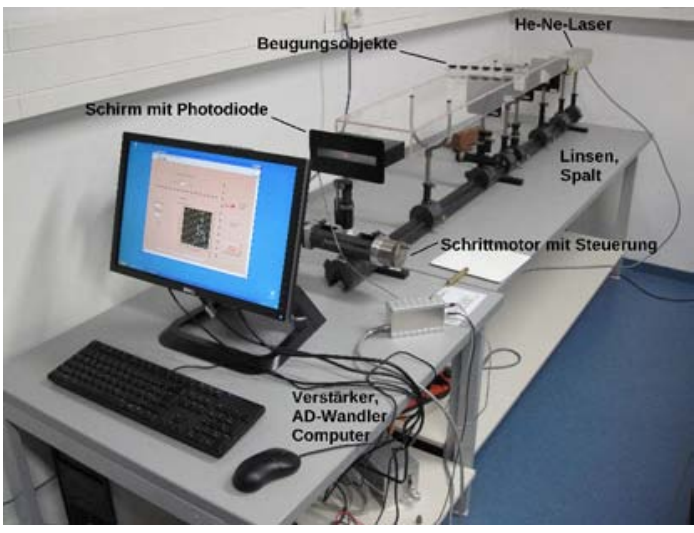

Bild 18.1: Der Versuch »Beugung und Interferenz von Laserstrahlung «. 
Bild 18.1 zeigt ein Foto des Versuches mit Zubehör: Optische Bank mit Helium-Neon-Laser ( $\lambda=632,8 \mathrm{~nm}$ ), Zerstreuungslinse, Sammellinse, Objekte: Spalt, Steg, Lochblende, Doppellochblende mit 3 verschiedenen Lochabständen, Gitter; Schrittmotor, Mattscheibe mit Fotodiode, Gleichspannungsverstärker $(0,5 \mathrm{mV}$ bis $10 \mathrm{~V})$, Analog-Digital-Wandler (ADC), Computer.

\subsection{Grundlagen}

\subsubsection{Laser}

Die Bezeichnung LASER steht für »Light Amplification by Stimulated Emission of Radiation «. Vom praktischen Standpunkt aus gesehen, ist der Laser einfach eine Lichtquelle, die einen eng gebündelten, intensiven Lichtstrahl aussendet. Das Licht des Lasers ist monochromatisch und kohärent, hat eine geringe Divergenz und ist meist polarisiert. Durch die Entstehungsgeschichte der Laserstrahlung hat sie meist auch noch ein spezielles Intensitätsprofil (TEM - Transversal Electromagnetic Modes). Laser gibt es in großer Vielfalt. Die Wellenlängen reichen vom infraroten bis zum ultravioletten Bereich 1 , die Leistungen variieren von Milliwatt bis zu Peta- und Exawatt. Bei den »klassischen« Lasern kommt der Lichtstrahl immer auf die gleiche Art und Weise zustande: Man benutzt ein Medium (z.B. Gase, Rubinkristalle, Neodymglas, Farbstoffe), das man mit Energie »vollpumpt«. Anschließend bringt man das Medium dazu, die gespeicherte Energie »stimuliert« in Form von Licht wieder abzugeben. Das Licht wird dann in einem Resonator aus Spiegeln zu einem Strahl gebündelt. Die Lichtquanten entstehen dadurch, dass ein Atom oder Molekül von einem angeregten Zustand in einen energieärmeren Zustand »fällt«. Die Anregungszustände können unterschiedliche Ursachen haben wie Elektronenanregung in der Hülle, Schwingungen oder Rotationen von Molekülen. Der Praktikumsversuch verwendet einen Helium-Neon-Gaslaser (He-Ne-Laser), um Beugungs- und Interferenzerscheinungen an einfachen geometrischen Objekten zu erzeugen und Intensitätsverteilungen quantitativ auszumessen, d.h. die Kohärenz und Monochromasie der Laser-Strahlung wird direkt ausgenutzt.

\section{Absorption, spontane und induzierte Emission}

Betrachtet man schematisch ein Atom mit zwei Energiezuständen $E_{1}$ und $E_{2}$, so kann man verschiedene Wechselwirkungen dieses Atoms mit elektromagnetischer Strahlung (in Form von Lichtquanten) betrachten.

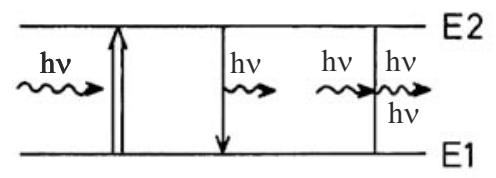

Absorption spontane induzierte Emission Bild 18.2: Absorption, spontane und induzierte Emission.

1. Die Absorption eines Lichtquants der Energie $E=h v=E_{2}-E_{1}$ regt das System aus dem tieferen Energiezustand $E_{1}$ in den höheren Zustand $E_{2}$ an (optischer Übergang).

1 Mittlerweile auch bis in den EUV (extended ultraviolet) und XUV (x-ray ultraviolet) und sogar den Röntgenbereich (FEL - Free Electron Laser) 
2. Der angeregte Zustand hat eine mittlere Lebensdauer, nach der er in den niedrigeren $\mathrm{Zu}$ stand zurückfällt und dabei ein Lichtquant der Energie $E=h v=E_{2}-E_{1}$ aussendet (Spontane Emission).

3. Ein vorhandenes Lichtquant der Energie $E=h v=\hbar \omega=E_{2}-E_{1}$ kann ein entsprechendes angeregtes Atom dazu veranlassen, unter Aussendung eines weiteren Quants $h v$ in den tieferen Zustand überzugehen(Induzierte Emission).

Im thermischen Gleichgewicht kann die Besetzung der beiden Energieniveaus durch eine Boltzmannverteilung beschrieben werden. Bezeichnet man die Anzahl der Atome im Zustand $E_{1}$ bzw. $E_{2}$ mit $N_{1}$ bzw. $N_{2}$, so ist das Verhältnis der Besetzungszahlen:

$$
\frac{N_{2}}{N_{1}}=\frac{e^{-E_{2} / k T}}{e^{-E_{1} / k T}}
$$

Bringt man dieses System in ein Strahlungsfeld, so kann man die Wahrscheinlichkeiten für die möglichen Übergänge wie folgt angeben: Die Absorption eines Quants ist der Besetzungszahl $N_{1}$ und der Dichte des Strahlungsfeldes $u(v)$ proportional, d.h. es gilt

$$
d N_{12}^{\text {Absorption }}=B_{12} \cdot u(v) \cdot N_{1} \cdot d t
$$

Die Zahl der spontanen Emissionen pro Zeiteinheit ist einfach der Besetzungszahl $N_{2}$ proportional, also

$$
d N_{21}^{\text {spontan }}=A_{21} \cdot N_{2} \cdot d t
$$

Die induzierte Emission ist analog zur Absorption proportional zur Besetzungszahl $N_{2}$ im angeregten Zustand und zur Strahlungsdichte $u(v), \mathrm{d}$. h.

$$
d N_{21}^{\text {induziert }}=B_{21} \cdot u(v) \cdot N_{2} \cdot d t
$$

Die Proportionalitätsfaktoren $B_{12}, A_{21}$ und $B_{21}$ nennt man Einstein-Koeffizienten. Sie sind ein Maß für die Übergangswahrscheinlichkeiten je Zeit- und Strahlungsdichteeinheit. Im Gleichgewicht werden sich die Übergänge in beiden Richtungen ausgleichen. Deshalb gilt der Zusammenhang

$$
d N_{12}^{\text {Absorption }}=d N_{21}^{\text {spontan }}+d N_{21}^{\text {induziert }} .
$$

Man kann auch das Verhältnis der Besetzungszahlen mit den Einstein-Koeffizienten angeben

$$
\frac{N_{2}}{N_{1}}=\frac{B_{12} \cdot u(v)}{A_{21}+B_{21} \cdot u(v)}
$$

Vergleicht man dieses Verhältnis $N_{2} / N_{1}$ mit der Boltzmannverteilung und löst nach der Strahlungsdichte $u(v)$ auf, so findet man die Gleichung der Planckschen Strahlungsdichteformel:

$$
u(v)=\frac{A_{21}}{B_{12} \cdot e^{h v / k T}-B_{21}}
$$


Die Einstein-Koeffizienten $B_{12}$ und $B_{21}$ sind in diesem Versuch gleich, was nichts anderes bedeutet, als dass das System Photonen in gleicher Weise abgibt wie aufnimmt. Absorption und erzwungene Emission sind also physikalisch äquivalente Prozesse.

\section{Inversion der Besetzungszahlen}

Der Laser als Lichtquelle setzt voraus, dass sich genügend Atome (oder Moleküle ...) im angeregten Zustand befinden (Anhand der Boltzmannverteilung kann man sich leicht davon überzeugen, dass das zunächst nicht der Fall ist, man setze z. B. T=300 K, $E_{2}-E_{1}=1 \mathrm{eV}$ als typische Werte ein). Durch Energiezufuhr von außen (»Pumpen«) müssen mehr Atome angeregt werden als es dem thermischen Gleichgewicht entspricht, während der tiefer liegende Zustand vergleichsweise gering besetzt ist. Man nennt diesen Vorgang Inversion der Besetzungszahlen.

Schwierigkeiten entstehen dadurch, dass beim optischen Pumpen nicht alle Atome denselben angeregten Zustand erreichen, sondern dass die aufgewandte Energie auf viele mögliche Anregungszustände verteilt wird. So gehen für die Emission einer bestimmten Frequenz Atome verloren. Ein zweites Problem liegt darin, dass die angeregten Atome normalerweise nur eine sehr kurze Lebensdauer (Größenordnung Nanosekunden) haben, und deshalb schnell durch spontane Emission verloren gehen. Dieses zweite Problem lässt sich umgehen, wenn man die Existenz so genannter metastabiler Zustände ausnutzt. Hier handelt es sich um angeregte Niveaus, deren Lebensdauern vergleichsweise lang sind, weil optische Übergänge zu tieferen Niveaus stark behindert oder verboten sind (»Auswahlregeln«).

So kommt man zu den Systemen, die man mit Drei-Niveaulaser bzw. Vier-Niveaulaser bezeichnet. Beim 3-Niveaulaser (Typisches Beispiel: Rubinlaser) können Atome in eine Reihe höher liegender Zustände (oder einen breitbandigen Zustand) angeregt werden, aus denen sie in einen metastabilen Zwischenzustand $E_{2}$ zerfallen. Hier können sich dann angeregte Atome ansammeln, bis sie durch induzierte Emission gemeinsam in den Grundzustand $E_{1}$ abgeregt werden. Der Nachteil liegt natürlich darin, dass der Laserübergang in den relativ dicht besetzten Grundzustand erfolgt, was zur Inversion der Besetzungszahlen ziemlich große Pumpleistungen erfordert. Beim 4-Niveaulaser umgeht man diesen Nachteil dadurch, dass der Laserübergang nicht in den Grundzustand $E_{1}$, sondern in ein darüber liegendes Niveau $E_{1}^{*}$ erfolgt. Wenn dieser Zustand kaum besetzt ist, ist eine Inversion der Besetzungszahlen von $E_{2}$ und $E_{1}^{*}$ leicht möglich.

\section{Resonatorbedingung}

Der Laser arbeitet erst, wenn die induzierte Emission nicht regellos erfolgt. In der Praxis erzeugt man den »Laserstrahl « dadurch, dass man das Lasermaterial in einen Resonator bringt. In der einfachsten Form besteht er aus zwei parallelen Spiegeln. Von dem Licht, das im Lasermedium entsteht, wird nur das senkrecht auf die Spiegel fallende ins Medium zurückgeworfen. Lichtquanten, die seitlich emittiert werden, gehen verloren. Das Licht, das zwischen den Spiegeln hinund her reflektiert wird, kann angeregte Atome wieder zur induzierten Emission bringen. Dieser Prozess führt schnell zu einer positiven Rückkopplung, d.h. der Lichtstrahl, der senkrecht auf die Spiegel trifft, wird immer stärker. Ist einer der Spiegel teildurchlässig, so tritt ein Teil des parallelen Laserstrahles aus. Der Laser ist also einem rückgekoppelten Verstärker vergleichbar, der die ihm durch Strahlung entzogene Energie durch phasenrichtige induzierte Emission wieder ergänzt. Im optischen Resonator werden nur bestimmte Eigenschwingungen (Moden) verstärkt. 
Die Bedingung entspricht der für stehende Wellen zwischen den Spiegeln, also

$$
l=n \cdot \frac{\lambda}{2}
$$

wenn man die Länge des Resonators mit $l$ bezeichnet (Diese Bedingung ist beim HeNe-Laser schärfer als etwa die Dopplerbreite der roten $632,8 \mathrm{~nm}$ Linie, d.h. man verstärkt mehrere Frequenzen innerhalb der Dopplerbreite!) [67].

\section{Der Helium-Neon-Laser}

Der HeNe-Laser arbeitet mit einem Gemisch aus etwa $88 \%$ Helium und $12 \%$ Neon unter geringem Druck (0,1 mbar). Durch eine Gasentladung regt man überwiegend die zahlreicheren Heliumatome an (Elektronische Anregung). Diese fallen dann in die metastabilen Helium-Zustände $2^{3} S$ und $2^{1} S$ zurück, aus denen kein optischer Übergang in den Grundzustand möglich ist (Drehimpuls-Auswahlregel bzw. Interkombinationsverbot). Die Energie dieser Zustände ist nun fast genau gleich der 2s- und 3s-Zustände von Neon, deshalb kann man diese Anregungsenergie durch Stöße auf die Neonatome übertragen. Aus den 2s- und 3s-Niveaus wäre ein Übergang in den Neon-Grundzustand denkbar, aber wegen dessen hoher Besetzung unerwünscht. Durch den passenden Partialdruck des Neongases werden die entsprechenden Quanten durch Absorption wieder eingefangen. Als Laser-Übergänge dienen im Neon die Übergänge von $3 \mathrm{~s}$ nach $3 p$ (infrarot, $3,39 \mu \mathrm{m}$ ), von $3 \mathrm{~s}$ nach $2 \mathrm{p}$ (rot $632,8 \mathrm{~nm}$ ) und von von $2 \mathrm{~s}$ nach $2 \mathrm{p}$ (infrarot $1,15 \mu \mathrm{m}$ ). Wegen der Aufspaltung dieser Terme sind zahlreiche Laserlinien möglich. Die dann erreichten Zustände $3 p$ und $2 p$ werden durch spontane Emission in die metastabilen 1s-Zustände abgebaut. Zurück in den Grundzustand gelangen die Ne-Atome durch Stöße mit der Rohrwand (Kleiner Rohrdurchmesser verursacht eine schnelle Entleerung dieser Zustände).

\subsubsection{Intensität}

Die Intensität einer Welle, also die Energie, die pro Zeiteinheit auf eine Einheitsfläche fällt, ist im Falle vom Licht gleichmäßig auf seine elektrischen und magnetischen Komponenten verteilt. Die Lichtgeschwindigkeit $c$ in Materie wird von deren Permittivität $\epsilon_{r} \epsilon_{0}$ und Permeabilität $\mu_{r} \mu_{0}$ bestimmt und beträgt:

$$
c=\frac{1}{\sqrt{\mu_{0} \cdot \mu_{r} \cdot \varepsilon_{0} \cdot \varepsilon_{r}}} .
$$

Außerdem kann man die Intensität der Welle $I$ durch ihre Energiedichte $d W / d V$ und die Geschwindigkeit im Medium (bzw. im Vakuum) ausdrücken:

$$
I=\frac{\frac{d W}{d t}}{d A}=\frac{c \cdot \frac{d W}{d t}}{\frac{d x}{d t} \cdot d A}=c \cdot \frac{d W}{d V} .
$$

Die Energiedichte des elektrischen Feldes ist gleich der des magnetischen Feldes

$$
W_{\mathrm{el}}=\frac{1}{2} \varepsilon_{0} \varepsilon_{r} \cdot E^{2}=\frac{1}{2} \mu_{0} \mu_{r} \cdot B^{2}=W_{\mathrm{magn}} .
$$


Die gesamte Intensität $I$ einer elektromagnetischen Welle ist also

$$
I=n c \epsilon_{0} E^{2}=\frac{E^{2}}{Z}=\sqrt{\frac{\epsilon_{0}}{\mu_{0}}} \sqrt{\frac{\epsilon_{r}}{\mu_{r}}} E_{0}^{2} .
$$

Diese Ableitung kann man auch über den Poynting Vektor $\vec{S}=\vec{E} \times \vec{H}$ führen. Ist die Intensität der von der Quelle abgestrahlten Energie fest, so ist die Intensität vom Medium abhängig, entweder ausgedrückt durch den Brechungsindex $n$ oder durch den Wellenwiderstand $Z$ des Mediums.

Im Versuch geht es um die Extrema der Intensität, um ihre Beugungswinkel (Positionen) und nicht um den absoluten Wert der Intensität; so eignet sich hier eine einfache Fotodiode, deren Fotospannung der Lichtintensität $I$ proportional ist.

\subsubsection{Beugung und Interferenz}

Um Beugungsfiguren zu berechnen, nimmt man einfallende ebene Wellen an, die durch eine Gleichung der Form

$$
\vec{E}=\overrightarrow{E_{0, c}} \cdot e^{i(k x-\omega t)}
$$

beschrieben werden können. $\overrightarrow{E_{0, c}}$ ist die komplexe Amplitude, $k$ die Wellenzahl und $\omega$ die Kreisfrequenz $\omega=2 \pi v$. Mit reeller Amplitude $\overrightarrow{E_{0}}$ und der Phase $\delta$ schreibt man die Wellengleichung in der Form

$$
\vec{E}=\overrightarrow{E_{0}} \cdot e^{i(k x-\omega t)} \cdot e^{i \delta}
$$

Um nun die Beugungsmuster in Abhängigkeit vom Beugungswinkel $I(\alpha)$ zu ermitteln, zerlegt man die vom Beugungsobjekt kommenden Lichtwellen in infinitesimale Teilwellen und integriert für jede Beugungsrichtung $\alpha$ phasenrichtig über das Objekt. Hier nehmen wir immer den Fall der Fraunhofer Beugung an, wo angenommen wird, das Lichtquelle und Beobachtungsschirm unendlich weit vom Beugungsobjekt entfernt sind (parallele Strahlen). Mit der Wellenlänge $\lambda$ des Lichtes und der geometrischen Abmessung $D$ des Objektes erhält man dann einfach, charakteristische Intensitätsverteilungen, die es gestatten Rückschlüsse auf die Objekte selbst zu ziehen. Im Versuch werden Steg, Lochblende, Doppellochblende, Spalt und Gitter behandelt. Die Intensitätsmuster werden jeweils beschrieben durch Gleichungen der Form

$$
I(\varepsilon)=I_{0} \cdot f(\varepsilon) \quad \text { mit: } \quad \varepsilon=\frac{\pi D \sin \alpha}{\lambda} .
$$

Die Variable $\varepsilon$ erspart etwas Schreibarbeit, und da die Winkel $\alpha$ meist sehr klein sind, kann $\sin \alpha$ durch $\alpha$ selbst ersetzt werden. Beim Doppelloch mit Lochabstand $D$ ergibt sich eine $\cos ^{2}(\varepsilon)$ Verteilung der Intensität, der aber noch das Beugungsmuster des Loches selbst überlagert wird.

Die jeweiligen Lösungen werden hier nur vereinzelt hergeleitet, aber alle angegeben. Die Kreisblende (Lochblende) wird explizit ausgerechnet, da sie mathematisch etwas anspruchsvoller ist. 


\section{Doppelloch}

Zwei punktförmige Löcher (Durchmesser $B \rightarrow 0$ ) haben einen Abstand $D$ und man beobachtet die Interferenzfigur dahinter, die sich auch den beiden Teilstrahlen 1 und 2 ergibt. Der Strahl 2

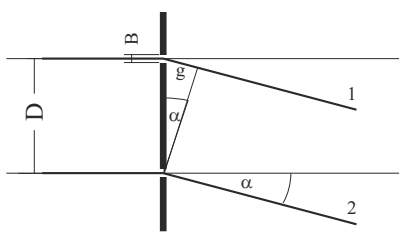

Bild 18.3: Beugung an zwei punktförmigen Öffnungen.

liefert zur resultierenden Feldstärke $\mathrm{E}_{r}$ den Beitrag $E_{0}$, der Strahl 1 liefert $E_{0} \cdot e^{i \delta}$. Die gesamte Feldstärke berechnet sich also zu

$$
E_{r}=E_{0} \cdot\left(1+e^{i \delta}\right)
$$

Den Gangunterschied $g$ der beiden Strahlen erhält man aus der Phase $\delta$ über

$$
\frac{2 \pi}{\delta}=\frac{\lambda}{g} \Rightarrow \delta=\frac{2 \pi g}{\lambda}=\frac{2 \pi D \alpha}{\lambda},
$$

wenn man die Näherung $\alpha \approx \sin \alpha \approx \frac{g}{D}$ benutzt. Die Intensität $\mathrm{I}_{r}$ ist $\left|E_{r}\right|^{2}=E_{r} \cdot E_{r}^{*}$, wobei $E_{r}^{*}$ die konjugiert komplexe Amplitude zu $E_{r}$ ist. Schreibt man

$$
\overrightarrow{E_{r}}=\overrightarrow{E_{0}} \cdot\left(1+e^{i \delta}\right)=\overrightarrow{E_{0}} \cdot\left(e^{-i \frac{\delta}{2}}+e^{i \frac{\delta}{2}}\right) \cdot e^{i \frac{\delta}{2}}=2 \cdot \overrightarrow{E_{0}} \cdot e^{i \frac{\delta}{2}} \cdot \cos \left(\frac{\delta}{2}\right)
$$

so wird

$$
I_{r}=4 \cdot E_{0}^{2} \cdot \cos ^{2}\left(\frac{\delta}{2}\right) \cdot\left|e^{i \frac{\delta}{2}}\right| .
$$

Nun ist $\left|e^{i \phi}\right|=1$, deshalb folgt für die Intensität

$$
I_{r}=4 \cdot E_{0}^{2} \cdot \cos ^{2}\left(\frac{\delta}{2}\right)=4 \cdot E_{0}^{2} \cdot \cos ^{2}\left(\frac{\pi D \alpha}{\lambda}\right) .
$$

Also erhalten wir als Lösung für das Doppelloch mit Lochabstand $D$ :

$$
I(\varepsilon)=I_{0} \cdot \cos ^{2}(\varepsilon) \quad \text { mit: } \quad \varepsilon=\frac{\pi D \alpha}{\lambda}
$$

\section{Beugung am Spalt der Breite D}

Man denkt sich den Spalt in Teile der Breite $\Delta \xi_{i}$ zerlegt. Aufintegriert ergibt dies als Lösung für die Intensität hinter einem Spalt der Öffnungsbreite $D$ : 


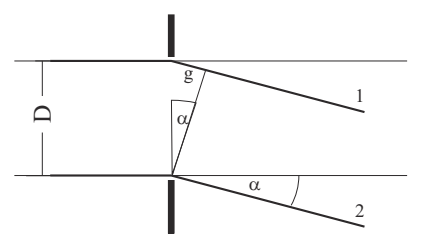

Bild 18.4: Beugung am Spalt.

$$
I_{r}=E_{0}^{2} \cdot\left(\frac{\sin (\varepsilon)}{\varepsilon}\right)^{2}
$$

\section{Beugung am Steg der Breite D}

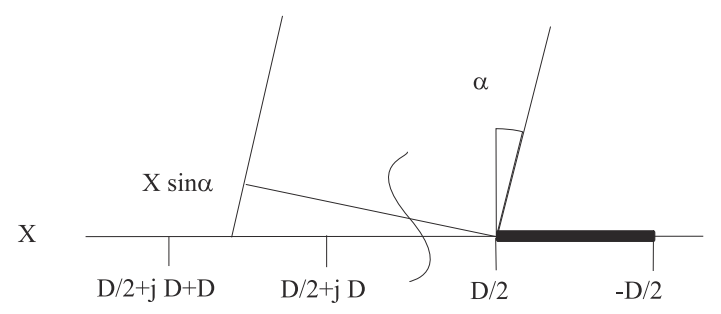

Hinter dem Steg überlagert sich die einfallende Welle $E_{0}$ mit einer um $\pi$ phasenverschobenen Welle $E_{0} \cdot e^{i \pi}$, so dass dort Auslöschung stattfindet. Nach dem Babinetschen Theorem erhält man daher dieselbe Intensitätsverteilung wie am Spalt. Der Phasensprung um $\pi$ wird optisch nicht wahrgenommen, weil das Auge nur Intensitäten wahrnehmen kann).

Die Zerlegung der Öffnung beim Steg kann zum Beispiel so erfolgen, dass man die Beiträge von Spalten der selben Größe des Stegs aufsummiert. Das Ergebnis für das elektrische Feld ist bis auf eine Phasenverschiebung von $\pi$ identisch mit dem für den Spalt. Sehr einfach kann man das Ergebnis auch ermitteln, in dem man bedenkt, dass die Superposition der elektrischen Felder des Spalts und des Stegs wie auch für jede anderen komplementären Objekte vollkommene Auslöschung ergeben muss (hinter einem unendlich ausgedehnten Schirm herrscht perfekte Dunkelheit):

$$
E_{\text {Spalt }}+E_{\text {Steg }}=0
$$

also ist die wahrgenommene Intensität hinter dem Steg exakt die gleiche wie beim Spalt. Selbstverständlich gibt es keine unendlich ausgedehnte Quelle monochromatischen parallelen kohärenten Lichtes. So ist das aufgenommene Beugungsspektrum nur näherungsweise das von einem Steg, vielmehr das eines Doppelspaltes mit viel kleinerer Stegbreite als Spaltbreite. Man kann sich leicht überzeugen, dass dieses Spektrum in das des Steges übergeht, je breiter das Lichtspot des Laser ist. Wichtig ist auch, dass der Steg rechts und links gleichmäßig bestrahlt wird 2

2 Natürlich ist unsere Lichtquelle nicht unendlich ausgedehnt, so dass dies hier nur beschränkt gilt, aber noch annehmbare Ergenisse liefert. 


\section{Beugung an einer Kreisblende mit dem Durchmesser D}

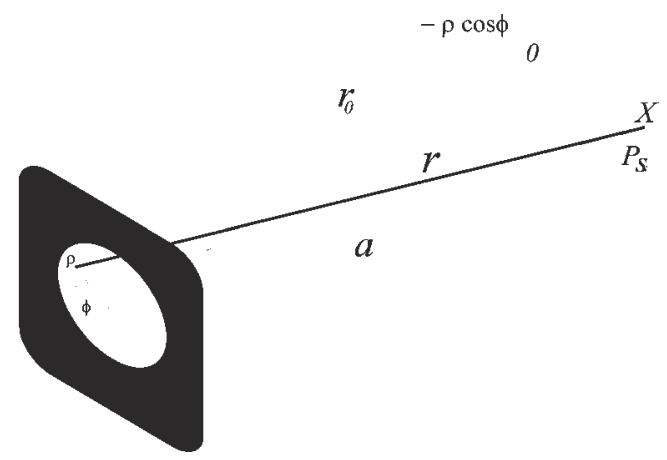

Bild 18.6: Beugung an der Kreisblende.

Das Loch der Kreisblende habe Durchmesser $D=2 R$. Sei $P$ ein Punkt auf der Kreisblende mit Polarkoordinaten $(\rho, \varphi)$ wie im Bild gezeigt. Sei $r$ der Abstand von Punkt $P$ zu einem Punkt $P_{s}$ auf dem Schirm, der sich um $x$ von dessen Mitte befindet. Weiter sei $a$ der Abstand zwischen dem Zentrum der Kreisblende und $P_{s}$ :

$$
\begin{aligned}
& r=\sqrt{r_{0}^{2}+(x+\rho \cos \varphi)^{2}+(\rho \sin \varphi)^{2}} \\
& a=\sqrt{r_{0}^{2}+x^{2}}
\end{aligned}
$$

dann ist auch $r=\sqrt{a^{2}+\rho^{2}+2 x \rho \cos \varphi}$. Der Radius der Kreisblende ist klein gegenüber dem Abstand vom Schirm $R \ll r_{0}$. Dann können Sie zeigen, dass man den Abstand $r$ mit

$$
r=a+\frac{x \rho \cos \varphi}{a}
$$

annähern kann. Gegenüber dem Strahl von der Mitte haben alle anderen Strahlen einen Gangunterschied von

$$
g(\rho, \varphi)=\frac{x \rho \cos \varphi}{a}=\rho \sin \alpha \cos \varphi
$$

Dann liefert das infinitesimale Flächenelement $\rho \mathrm{d} \rho \mathrm{d} \varphi$ der Kreisblende zum gesamten Feld einen Beitrag

$$
d E=E_{0} \cdot \frac{\rho \mathrm{d} \rho \mathrm{d} \varphi}{\pi R^{2}} \cdot \mathrm{e}^{i \frac{2 \pi}{\lambda} \rho \sin \alpha \cos \varphi}
$$

Mit der Integration über die ganze Kreisblende erhält man das Gesamtfeld zu

$$
E=\frac{E_{0}}{\pi R^{2}} \int_{0}^{R}\left(\int_{0}^{2 \pi} \mathrm{e}^{i \frac{2 \pi}{\lambda} \rho \sin \alpha \cos \varphi} \mathrm{d} \varphi\right) \rho \mathrm{d} \rho
$$




$$
\begin{aligned}
& =\frac{2 \cdot E_{0}}{R^{2}} \int_{0}^{R}\left(\frac{1}{2 \pi} \cdot \int_{0}^{2 \pi} \mathrm{e}^{i \frac{2 \pi}{\lambda} \rho \sin \alpha \cos \varphi} d \varphi\right) \rho d \rho \\
& =\frac{2 E_{0}}{R^{2}} \int_{0}^{R} J_{0}\left(\frac{2 \pi}{\lambda} \rho \sin \alpha\right) \rho d \rho \\
& =\frac{2 E_{0}}{R^{2}\left(\frac{2 \pi}{\lambda} \sin \alpha\right)^{2}} \cdot \int_{0}^{\left.\frac{2 \pi}{\lambda} R \sin \alpha\right)} J_{0}\left(\frac{2 \pi}{\lambda} \rho \sin \alpha\right) \cdot\left(\frac{2 \pi}{\lambda} \rho \sin \alpha\right) \mathrm{d}\left(\frac{2 \pi}{\lambda} \rho \sin \alpha\right) \\
& =-2 E_{0} \frac{J_{1}\left(\frac{2 \pi}{\lambda} R \sin \alpha\right)}{\left(\frac{2 \pi}{\lambda} R \sin \alpha\right)}:=-2 E_{0} \frac{J_{1}(\epsilon)}{\epsilon}
\end{aligned}
$$

wobei man sich folgende Eigenschaft der zylindrischen Bessel-Funktionen $J_{0}$ und $J_{1}$ zu Nutze gemacht hat:

$$
\int_{0}^{\epsilon} \xi J_{0}(\xi) d \xi=-\epsilon J_{1}(\epsilon)
$$

Das Intensitätsprofil im Falle der Kreisblende mit Durchmesser $D=2 R$ ist mit $\varepsilon=\frac{\pi D \sin \alpha}{\lambda}$ also

$$
I=I_{0} \cdot\left(\frac{J_{1}(\epsilon)}{\epsilon}\right)^{2}
$$

Wie erwartet, hat man ein ähnliches Intensitätsprofil wie beim Spalt, nur dazu rotationssymmetrisch, also mit einem hellen Kreis in der Mitte und konzentrische Ringe, deren Helligkeit stark mit dem Radius abnimmt. Die Bessel-Funktion $J_{1}$ ist in den üblichen Programmen implementiert. Die Maxima und Minima der Funktion $\left(\frac{J_{1}(\epsilon)}{\epsilon}\right)^{2}$ haben die in Tabelle 18.1 aufgeführten Werte. Der Zahlenwert 1,22 des ersten Minimums dürfte Ihnen schon öfter aufgefallen sein (z.B.

Tabelle 18.1: Extrema der Besselfunktion $J_{1}(\varepsilon)$.

\begin{tabular}{crrrrrrr}
\hline Extremum & $I_{\min _{1}}$ & $I_{\max _{1}}$ & $I_{\min _{2}}$ & $I_{\max _{2}}$ & $I_{\min _{3}}$ & $I_{\max _{3}}$ & $I_{\min _{4}}$ \\
\hline$\epsilon / \pi$ & 1.2197 & 1.6347 & 2.2331 & 2.6793 & 3.2383 & 3.6987 & 4.2411 \\
\hline
\end{tabular}

Auflösungsvermögen). Nun haben Sie gesehen wie dieser Zahlenwert zustande kommt.

\section{Beugung am Gitter}

Das Gitter habe $N$ Spalte der Breite $D$. Die Stegbreite zwischen den Spalten sei $S$. Die Gitterkonstante wäre dann $g=\frac{1}{D+S}$ (d.h. Zahl der Spalte pro Längeneinheit). Zur Berechnung nummeriert man die Spalte von 0 bis $(\mathrm{N}-1)$ durch. Der $v$-te Spalt beginnt dann bei $v \cdot(D+S)$ und endet bei 


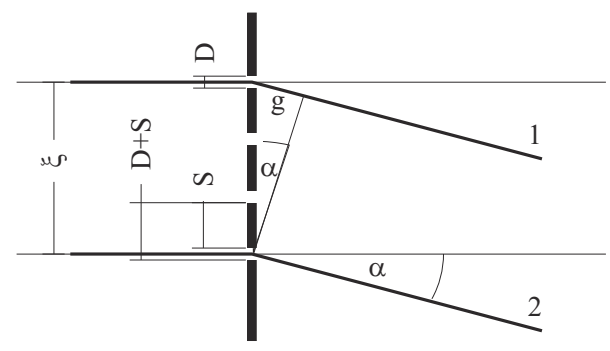

Bild 18.7: Beugung am Gitter.

$v \cdot(D+S)+D$ (siehe Bild). Nach etwas Rechnung ergibt sich die Intensität $I_{r}$ hinter dem Gitter als:

$$
I_{r}=E_{0}^{2} \cdot \underbrace{\left(\frac{\sin \left(\frac{\pi \alpha D}{\lambda}\right)}{\frac{\pi \alpha D}{\lambda}}\right)^{2}}_{\text {Spaltfunktion }} \cdot \underbrace{\left(\frac{\sin (N \varepsilon)}{\sin (\varepsilon)}\right)^{2}}_{\text {Gitterfunktion }}
$$

wo die Terme Gitterfunktion und Spaltfunktion definiert sind. Da $\varepsilon=\frac{\pi \alpha(D+S)}{\lambda}$ größer ist als $\frac{\pi \alpha D}{\lambda}$, wird die Helligkeitsverteilung am Gitter im Wesentlichen durch die Gitterfunktion bestimmt. Die Spaltfunktion moduliert die Intensitätsverteilung zusätzlich.

Als Beispiel wird hier für $D=2 S$ und $N=4$ die Intensitätsverteilung berechnet. Man hat also die Beziehung

$$
\varepsilon=\frac{\pi \alpha(D+S)}{\lambda}=\frac{3}{2} \cdot \frac{\pi \alpha D}{\lambda}
$$

und eine Intensitätsverteilung der Form

$$
I_{r}=E_{0}^{2} \cdot\left(\frac{\sin \left(6 \frac{\pi \alpha D}{\lambda}\right)}{\sin \left(\frac{3}{2} \frac{\pi \alpha D}{\lambda}\right)}\right)^{2} \cdot\left(\frac{\sin \left(\frac{\pi \alpha D}{\lambda}\right)}{\frac{\pi \alpha D}{\lambda}}\right)^{2}
$$

Man findet Hauptmaxima dieser Verteilung an den Stellen, an denen $\frac{\pi \alpha D}{\lambda}=\frac{2}{3} n \pi$ für $n=$ $0,1,2, \ldots$ wird. Für $n$ sind Vielfache von 3 auszuschließen, weil dann der Zähler der Spaltfunktion Null ist. Das Hauptmaximum für $n=3$ tritt deshalb gar nicht auf! Nebenmaxima findet man, wenn der Zähler der Gitterfunktion Maxima hat, also wenn $\frac{\pi \alpha D}{\lambda}=\frac{1}{6}\left(m-\frac{1}{2}\right) \pi(m=1,2,3, \ldots)$ wird. Diese Situation ist in Bild 18.8 dargestellt.

\subsubsection{Versuchsaufbau}

Der Laser liefert ein schwach divergentes, kohärentes Lichtbündel, dessen Durchmesser am Laserausgang $1 \mathrm{~mm}$ beträgt. Der Divergenzpunkt liegt $1 \mathrm{~m}$ vor dem Laserausgang. Die gesamte Strahlungsleistung des Lasers beträgt etwa $1 \mathrm{~mW}$, was auf den Strahl mit $1 \mathrm{~mm}$ Durchmesser eine Leistungsdichte (Irradianz) von etwa $127 \mathrm{~mW} / \mathrm{cm}^{2}$ ergibt. Zum Vergleich: Die gesamte Strahlungsdichte der Sonne auf der Erde oberhalb der Erdatmosphäre beträgt $136 \mathrm{~mW} / \mathrm{cm}^{2}$. Der 
a)

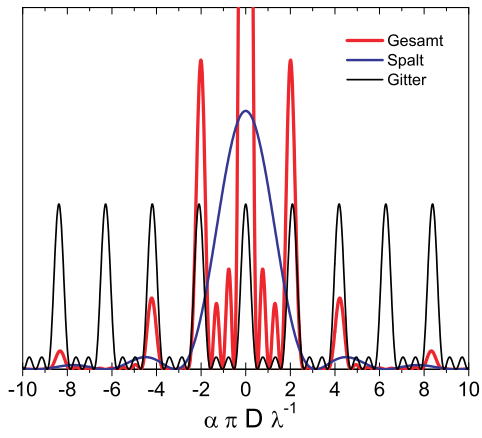

b)

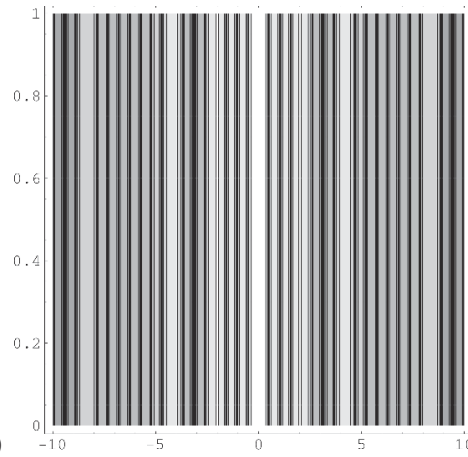

Bild 18.8: Intensitätsverteilung für das Beispielgitter mit $N=4$, links als Funktion, rechts als Intensitätskontur.

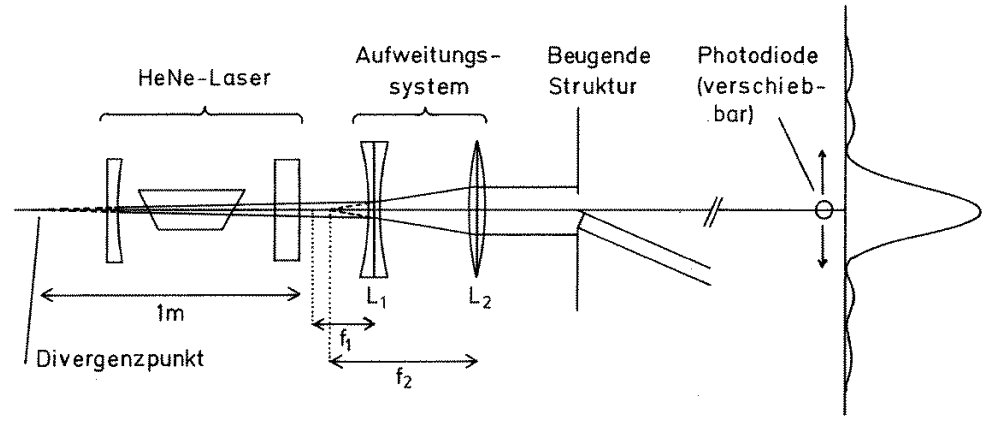

Bild 18.9: Strahlengang für den Versuch »Beugung mit Laserstrahlung«

Umgang mit dem Laser ist sehr gefährlich, wenn man in den direkten oder durch einen spiegelnden Gegenstand umgelenkten Laserstrahl blickt 3 Die verwendeten Linsen haben eine Brennweite von $f_{\text {konkav }}=-9 \mathrm{~cm}$ bzw. $f_{\text {kovex }}=+30 \mathrm{~cm}$. Ihre optimalen relativen Positionen können einfach berechnet werden, wenn man davon ausgeht, dass für die meisten Objekte ein Lichtspot von ca. $3 \mathrm{~mm}$ nicht zu klein wäre. Es sollte für alle Objekte ein homogener und symmetrischer Strahl über ihre gesamten Objektfläche (Durchlassbereich) eingestellt werden. Die Intensität wird mit einer Fotodiode vermessen, deren Fotospannung über einen Verstärker und AnalogDigital-Konverter (ADC) in den Computer eingelesen wird 4 Der Computer steuert über einen Schrittmotor die Position der Fotodiode, und somit den Beugungswinkel.

3 Der verwendete He-Ne-Laser hat Laserklasse 2. Die Laserschutzbestimmungen liegen im Praktikum aus.

4 Die Beugungsmuster haben eine hohe Dynamik, d.h. eine über mehrere Größenordnungen variierende Intensität. Dies ist mit solchen linearen Messgeräten meist nicht einfach zu erfassen. Das Auge hat für die Intensitätsmessung bereits eine Art Logarithmusfunktion eingesetzt kann deshalb seine hohe Dynamik erreichen. 


\subsection{Fragen}

Machen Sie sich bereits vor dem Versuch die folgenden Aspekte klar:

1. Wie entstehen stehende Wellen?

2. Wie wird im Laser Energie in Form von Licht verstärkt? Welche Rolle spielen dabei metastabile Zustände und stehende Wellen?

3. Welche Größenordnung hat die Kohärenzlänge des erzeugten Lichtes beim He-Ne Laser?

4. Weshalb soll der Gasresonator lang und dünn sein?

5. Wie wird das Laser-Licht nur sehr schwach divergent gemacht?

6. Welche Rolle spielt der Grenzwinkel für Polarisation in einem Laser?

7. Welche Energiedichte haben die zwei Komponenten der Lichtwelle und was ist die Intensität einer Welle?

8. Leiten Sie bitte die erwarteten Intensitätsprofile der vorhandenen Objekte her, mit der Annahme von parallel einfallendem Licht und Beobachtung im Unendlichen bzw. auf der Brennebene einer Sammellinse.

9. Bei welchen Objekten werden Sie besonders dafür sorgen müssen, dass der Lichtfleck möglichst gleichmäßig die Durchlassbereiche bestrahlt?

\subsection{Weiterführendes}

1. Bei einer Doppellochblende: bis zu welchem Abstand der Zentren unterscheidet sich die ringförmige Beugungsfigur nicht von der der einfachen Lochblende?

2. Welches Maximum erster Klasse wird von der Spaltmodulation bei Gittern mit Verhältnis Spaltbreite/Stegbreite $D / B=3 / 4$ und $D / B=3 / 2$ unterdrückt?

3. Was ändert sich am Spektrum, wenn die Diode eine endliche Breite und eine endliche Höhe hat?

\subsection{Durchführung}

Das Gelingen dieses Versuchs hängt nicht nur von der Aufnahme der Spektren ab (die ja sowieso vom Rechner übernommen wird), sondern auch davon, wie sauber Sie mit der Optik umgehen. Fassen Sie die Objekte nur an ihrer Fassung an 5 . Bitte gehen Sie auch entsprechend vorsichtig und sauber mit den Linsen um.

1. Computer einschalten, Windows starten und anmelden (User=»laser«, kein Password). Die LABVIEW-Applikation »LaserSteuerung « auf dem Desktop starten; dies führt automatisch eine Referenzfahrt des Motors aus und setzt dessen Position auf 0. Laser einschalten. Der Einschalter des Lasers ist der Drehschalter (Schlüssel) vorn.

2. Vorbereitung: Mit der Zerstreuungslinse und der Sammellinse weiten Sie bitte das Lichtbündel auf (wenn es für das jeweilige Objekt überhaupt nötig ist). Man überlegt sich vor

5 Das Fett Ihrer Finger bindet Schmutz an die Öffnungen der Objekte, deren Dimensionen hierdurch stark verfälscht werden. 
dem Versuch mit Hilfe des Strahlengangs, wie die Linsen aufgestellt werden müssen. Entfernen Sie das Objekt aus dem Strahlengang und überprüfen Sie, ob die Strahlen parallel sind und das gesamte Strahlenbündel für alle Objekte diese ausreichend breit, homogen, symmetrisch ausleuchtet.

3. Beobachtung: Sagen Sie voraus, welche Beugungsmuster Sie für die einzelnen Beugungsobjekte erwarten. Stellen Sie die Objekte in den Strahlengang und kommentieren Sie die auf den Schirm projizierten Muster. Beobachten Sie zum Beispiel die Doppellochblenden mit verschiedenen Abständen zwischen den Löchern. Überlegen Sie, welches Verhältnis zwischen Spaltbreite und Stegbreite das gerade beobachtete Gitter haben soll, je nachdem welches Maximum erster Ordnung nicht vorhanden ist.

4. Nun beginnen Sie mit den Messungen des Intensitätsverlaufs von:
a. Spalt
b. Steg
c. Kreisblende
d. einer Doppellochblende
e. einem Gitter.

Der Abstand zwischen Objekt und Diode muss möglichst groß sein und gemessen werden. Die Aufnahmen selbst sind computergesteuert. Das Messprogramm lässt sich einfach bedienen und wird vom Desktop gestartet (s. Punkt1). Anzugeben sind u.a. der Messbereich für das Beugungsmuster, welchen die Diode abfahren soll (dieser wird in Schritten des Motors angegeben, 400 Schritte entsprechen $1 \mathrm{~mm}$ ) und der Spannungsbereich, der vom ADC digitalisiert werden soll (je nach Intensität des Lichtes $0 \ldots 1 \mathrm{~V}$ und $0 \ldots 10 \mathrm{~V}$ ). Stellen Sie sicher,

a. dass Sie symmetrische Spektren bis zum vierten Neben-Minimum links und rechts vom Hauptmaximum aufnehmen. Beim Gitter und bei der Doppelkreisblende genügt es bis zum ersten Neben-Maximum der Spalt- bzw. Kreisblende-Funktion, bei der Kreisblende sind die Maxima höherer Ordnung u.U. schwer zu identifizieren 6 . Fahren Sie die Diode manuell so weit nach links bzw. rechts, dass die Fotodiode für das betrachtete Beugungsobjekt am Rand des aufzunehmenden Bereichs liegt. Die manuelle Steuerung des Motors geschieht über relative Bewegungen im oberen Teil des Bedienfensters. Notieren Sie sich diese beiden Positionen, die im Bedienfenster rechts angezeigt werden.

b. dass die Positionen der Extrema deutlich zu bestimmen sind. Die Maxima größerer Ordnung haben ziemlich schwache Intensitäten. Andererseits hat der ADC »nur « 4096 Kanäle, was dessen Auflösung entsprechend begrenzt; die falsche Wahl des Messbereichs führt also zu einem Verlust in der Auflösung oder zu einem Übersteuern des ADC. Daher ist es erforderlich das Spektrum zweimal aufzunehmen, einmal im Bereich des Hauptmaximus, und einmal im Bereich der Nebenmaxima (zu beiden 
Seiten!). Wählen Sie jeweils den optimalen Spannungsbereich im rechten Teil des Bedienfensters aus, wo auch der aktuelle Messwert der Fotospannung abgelesen werden kann 7

Die Messung starten Sie in der Applikation unten links. Geben Sie eine Schrittweite ein, die eine möglichst hohe Auflösung des Spektrums ermöglicht (auf jeden Fall unter 50 Schritte). Die Messung ist völlig computergesteuert. Das aufgenommene Spektrum wird in eine Datei gespeichert, dabei geben Sie einen eindeutigen Namen an (ganz unten im Bedienfenster). Mit Hilfe eines zweiten Programmes (gnuplot) können Sie die Spektren darstellen, um die Messung zu überprüfen. Es empfiehlt sich dies sofort nach der jeweiligen Messung zu tun.

Bei jedem Objektwechsel überprüfen Sie bitte, dass dieses gleichmäßig bestrahlt wird.

5. Übertragen Sie die Dateien auf einen USB-Stick oder schicken sich diese per Email. Ihr(e) Betreuer(in) erhält hiervon am Versuchstag eine Kopie, was als Teil des Messprotokolls gilt. Die übrigen Angaben (welche Datei zu welchem Beugungsobjekt gehört, Konversionsfaktor (Schritte/mm), Abstand Objekt-Diode) sollten auf einem separaten Blatt notiert werden und werden wie ein konventionelles Messprotokoll behandelt.

\subsection{Angaben}

Die benötigten Größen der Objekte sind in Tabelle 18.2 aufgeführt. Bitte überprüfen Sie diese dennoch anhand des am Messplatz ausgelegten Korrekturblattes. Diese müssen im Messprotokoll notiert werden.

Tabelle 18.2: Geometrische Größenangaben der Beugungsobjekte für die drei Versuchsaufbauten beim Versuch »Laser«. Bitte achten Sie auf eventuelle Korrekturangaben an der Apparatur.

\begin{tabular}{llrrrrr}
\hline Objekt & Angabe & Aufbau 1 & Aufbau 2 & Aufbau 3 & Aufbau 4 & Aufbau 5 \\
\hline Spalt & Breite $D$ & $237 \mu \mathrm{m}$ & $225 \mu \mathrm{m}$ & $250 \mu \mathrm{m}$ & $170 \mu \mathrm{m}$ & $190 \mu \mathrm{m}$ \\
Steg & Breite $D$ & $195 \mu \mathrm{m}$ & $195 \mu \mathrm{m}$ & $200 \mu \mathrm{m}$ & $180 \mu \mathrm{m}$ & $180 \mu \mathrm{m}$ \\
Lochblende & Durchmesser $D$ & $200 \mu \mathrm{m}$ & $200 \mu \mathrm{m}$ & $200 \mu \mathrm{m}$ & $180 \mu \mathrm{m}$ & $190 \mu \mathrm{m}$ \\
Doppelloch (nah) & Abstand $D$ & $490 \mu \mathrm{m}$ & $500 \mu \mathrm{m}$ & $500 \mu \mathrm{m}$ & $430 \mu \mathrm{m}$ & $430 \mu \mathrm{m}$ \\
& Durchmesser $B$ & $210 \mu \mathrm{m}$ & $210 \mu \mathrm{m}$ & $200 \mu \mathrm{m}$ & $190 \mu \mathrm{m}$ & $180 \mu \mathrm{m}$ \\
Doppelloch (mittel) $)$ & Abstand $D$ & $700 \mu \mathrm{m}$ & $1000 \mu \mathrm{m}$ & $700 \mu \mathrm{m}$ & $830 \mu \mathrm{m}$ & $840 \mu \mathrm{m}$ \\
& Durchmesser $B$ & $210 \mu \mathrm{m}$ & $210 \mu \mathrm{m}$ & $200 \mu \mathrm{m}$ & $180 \mu \mathrm{m}$ & $180 \mu \mathrm{m}$ \\
Doppelloch (fern) & Abstand $D$ & $1500 \mu \mathrm{m}$ & $1500 \mu \mathrm{m}$ & $1500 \mu \mathrm{m}$ & $1270 \mu \mathrm{m}$ & $1260 \mu \mathrm{m}$ \\
& Durchmesser $B$ & $200 \mu \mathrm{m}$ & $140 \mu \mathrm{m}$ & $200 \mu \mathrm{m}$ & $180 \mu \mathrm{m}$ & $180 \mu \mathrm{m}$ \\
Gitter & Spaltbreite $D$ & $210 \mu \mathrm{m}$ & $210 \mu \mathrm{m}$ & $175 \mu \mathrm{m}$ & $160 \mu \mathrm{m}$ & $170 \mu \mathrm{m}$ \\
& Stegbreite $S$ & $280 \mu \mathrm{m}$ & $280 \mu \mathrm{m}$ & $110 \mu \mathrm{m}$ & $250 \mu \mathrm{m}$ & $250 \mu \mathrm{m}$ \\
\hline
\end{tabular}

7 Dabei können Sie sich bei Bedarf auch mit dem Verstärker behelfen, der von 1x auf 3x umgeschaltet werden kann. 


\subsection{Auswertung}

Ziel der Auswertung ist die Berechnung der charakteristischen Größen der Objekte, bzw. umgekehrt auch der Wellenlänge des Laserlichtes. Betrachten Sie den Versuch als aus 5 verschiedenen kleineren Versuchen bestehend: Beugung am Spalt, Beugung am Steg, Beugung an der Kreisblende, Beugung an der Doppelkreisblende und Beugung am Gitter. Werten Sie für jedes Objekt getrennt folgendermaßen aus:

1. Berechnen Sie für jedes Extremum (nicht nur die Minima oder die Maxima) die relativen Positionen der Extrema bezüglich des Hauptmaximums (HM). Kontrollieren Sie jetzt, dass diese links und rechts vom HM in gewissen Grenzen übereinstimmen, ist es nicht der Fall, dann überprüfen Sie erst die Position des HM und auch der Extrema. Korrigieren Sie sie falls nötig und berechnen Sie die relativen Positionen erneut. Machen Sie eine Tabelle mit den absoluten Positionen der Extrema, den relativen, den entsprechenden Werten von $\sin \alpha$. Der Winkel $\alpha$ ergibt sich aus Schirmabstand $l$ und Position des Schrittmotors $x$ zu $\alpha=$ $\arctan (x / l)$. Geben Sie die jeweilige Ordnung und den entsprechenden Wert von $\epsilon / \pi$ an.

2. Tragen Sie $\sin \left(\alpha_{i}\right)$ gegen $\frac{\epsilon_{i}}{\pi}$ auf. Die Messwerte sollten auf einer Gerade liegen. An dieser Stelle können Sie einen übersehenen Ausreißer korrigieren oder beseitigen. Berechnen Sie die Korrelation der Werte (den empirischen Korrelationskoeffizienten $r$ ), die Regressionsgerade mit den enstprechenden Fehlern der Parameter) und tragen Sie sie samt Gleichung in den Grafen ein.

3. Aus der Steigung der Regressionsgeraden ermitteln Sie die charakteristische Größe des Beugungsobjektes mit Vertrauensbereich (Fehlerfortpflanzung).

4. Vergleichen Sie Ihr Ergebnis mit dem erwarteten Wert; diskutieren Sie mögliche Fehlerquellen.

5. Für das Objekt bei dem die beste Korrelation zwischen $\sin \alpha_{i}$ und den entsprechenden $\epsilon_{i} / \pi$ vorliegt $\left(r^{2} \approx 0.999\right)$ nehmen Sie stattdessen seine Größe als bekannt an und bestimmen Sie daraus die Wellenlänge des optischen Übergangs des He-Ne-Lasers. Das Ergebnis muss mit dem entsprechendem Vertrauensbereich angeben werden. Vergleichen Sie Ihr Ergebnis mit dem Literaturwert und diskutieren Sie die durch Beugungsmessungen erreichbare Genauigkeit.

\subsection{Bemerkungen}

Die bei den Objekten angegebenen charakteristischen Größen wurden über ein Mikroskop vermessen, können sich jedoch mit der Zeit verändern. Überprüfen Sie bitte die jeweilige Aktualisierung an Ihrem Messplatz. 


\section{Die spezifische Elektronenladung}

Der Quotient aus Ladung $e$ und Masse $m_{e}$ des Elektrons wird spezifische Elektronenladung $e / m_{e}$ genannt. Ihre Kenntnis ist für viele Berechnungen notwendig, insbesondere bei bewegten Ladungen in elektrischen und magnetischen Feldern. Diese Naturkonstante $e / m_{e}$ soll hier bestimmt werden. Erstmals gelang dies Emil Wiechert (1861-1928) in Göttingen (Geophysik) aus der Ablenkung von Kathodenstrahlen fast gleichzeitig mit J.J. Thomson, wobei Letzterer dafür später den Nobelpreis erhielt.

Der Versuch an sich ist relativ einfach und kurz, deshalb soll Wert auf das Aufspüren systematischer Fehler gelegt werden.

\subsection{Stichworte}

Spezifische Elektronenladung, Elektronenstrahlröhre, Helmholtzspule, Lorentzkraft, homogenes Magnetfeld, Ablenkung von Elektronen im elektrischen Feld, Massenseparation.

\subsection{Literatur}

NPP; Walcher; Dem-2; Gerthsen; BS-2; Geschke; Schülerduden, »Die Physik«, Bibliografisches Institut Mannheim [61]; Dorn/Bader, »Physik in einem Band «, Hermann Schroedel Verlag, Hannover [15];

\subsection{Zubehör}

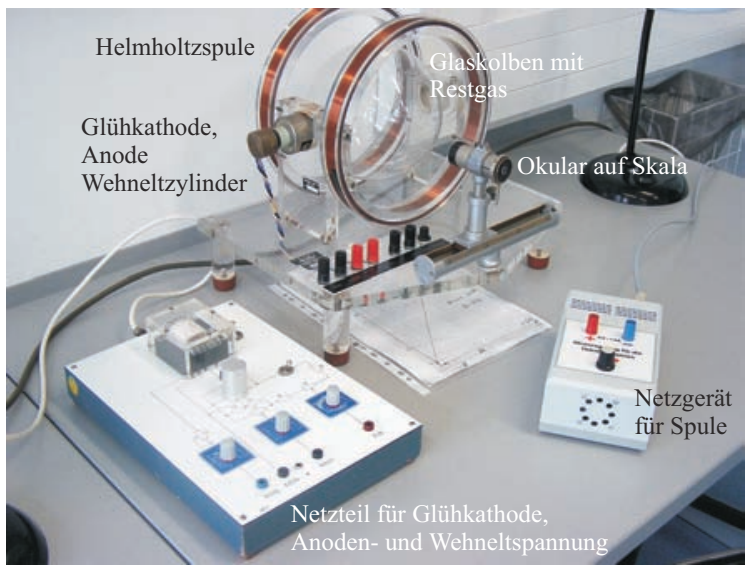

Bild 19.1: Der Versuch »Spezifische Elektronenladung «. 
Bild 19.1zeigt ein Foto des Versuches mit Zubehör: Fadenstrahlrohr mit Helmholtzspule und verschiebbarer Ablesevorrichtung, Steuerung für Fadenstrahlrohr (im wesentlichen Netzgerät für Kathodenheizung, Wehneltzylinder und Anodenspannung), Netzgerät mit regelbarem Strom für die Helmholtz-Spule, 2-3 Multimeter.

\subsection{Grundlagen}

Experimentell bedient man sich in den meisten Fällen der Helmholtz-Spule zur Erzeugung eines homogenen Magnetfeldes. Dies wurde im Versuch »Magnetfeld von Spulen« in Band 1 der Praktikumsanleitung [26] genauer beschrieben und untersucht.

Elektronen werden durch Glühemission erzeugt und mit einer elektrischen Spannung beschleunigt. Im Magnetfeld werden sie durch die Lorentzkraft auf eine Kreisbahn gezwungen.

\subsection{Fragen}

1. Fadenstrahlröhre mit Elektronenkanone:

a. Erklären Sie Aufbau und Funktionsweise einer Elektronenkanone mit Wehneltzylinder. Wie sieht ein Schaltplan dazu aus?

b. Welche Geschwindigkeit hat ein Elektron nach dem Durchlaufen der Beschleunigungsspannung $U_{B}$ ?

c. Wieso kann der Wehneltzylinder nur begrenzten Erfolg haben?

d. Wieso ist der Elektronenstrahl sichtbar (»Fadenstrahlen «)?

e. Elektronen stoßen sich aufgrund gleicher negativer Ladung ab. Wieso verschmiert der Elektronenstrahl trotzdem nicht zur Unkenntlichkeit (Gasfokussierung; Kanal aus Ionen)?

2. Bewegte Ladungen in magnetischen Feldern:

a. Welche Kraft $\vec{F}$ wirkt auf eine mit der Geschwindigkeit $\vec{v}$ bewegte Ladung in einem beliebigen magnetischen Feld $\vec{B}$ (Lorentzkraft)?

b. Wie lässt sich die Bahn eines Elektrons in einem homogenen Magnetfeld beschreiben? Welche Vereinfachung ergibt sich, wenn man annimmt, dass die Anfangsgeschwindigkeit $\overrightarrow{v_{0}}$ senkrecht zum magnetischen Feld $\vec{B}$ steht?

3. Ladungen in elektrischen Feldern:

a. Welche Kraft $\vec{F}$ wirkt auf eine Ladung in einem beliebigen elektrischen Feld $\vec{E}$ ? Wie lässt sich die Bahn eines Elektrons beim Durchfliegen eines Plattenkondensator bei parallelem Einschuss relativ zu den Platten beschreiben?

4. Braunsche Röhre/(Kathodenstrahl-)Oszilloskop:

a. Skizzieren Sie Aufbau (Skizze) und Funktionsweise einer Braunschen Röhre.

b. Welche Spannung $U_{x}(t)$ mit welcher Frequenz muss an den horizontalen Ablenkkondensator angelegt werden, um eine beliebige periodische Spannung $U(t)$ mit der Periodendauer $T$ auf dem Bildschirm darstellen zu können? 


\subsection{Durchführung}

\subsubsection{Hinweise zur Durchführung}

- Die Spannung am Wehneltzylinder sollte man sich mit einem Messgerät anzeigen lassen.

- Zur Schonung der Röhre müssen die Potenziometer für Kathodenheizung und Anodenspannung beim Einschalten auf Null stehen. Die Heizung muss langsam erhöht werden und die Anodenspannung soll erst eingeschaltet werden, wenn der Kathodenzylinder rotglühend ist.

- Der Spulenstrom darf 1 A nicht überschreiten.

- Man achte auf korrekte Justierung der Ablesevorrichtung (Höhe!).

- Normalerweise wird bei jeder Bestimmung des Durchmessers $d$ die linke und rechte Begrenzung des Kreises gemessen. Man kann sich auch überlegen, ob es ausreicht, eine Begrenzung (in unseren Fall die linke) sehr genau zu messen und als fest anzunehmen. Dabei ist in Betracht zu ziehen, dass die Fadenstrahlröhre nicht völlig fest mit dem Gestell verbunden und somit insbesondere auch eine Verschiebung relativ zur Ablesevorrichtung möglich wäre (z.B. beim Verschieben der Apparatur, oder bei Erschütterungen).

- Man achte auf Konstanz von Anodenspannung und Spulenstrom, gegebenenfalls ist nachzuregeln.

\subsubsection{Durchführung}

1. Die spezifische Elektronenladung wird bestimmt aus den eingestellten Parametern Beschleunigungsspannung $U_{B}$ und Spulenstrom $I$, sowie dem gemessenen Wert des Durchmessers $d=d\left(U_{B}, I\right)$. Vor dem Einschalten der Apparatur die »Hinweise zur Durchführung « beachten!

2. Zunächst überprüfe man in einem groben Raster (z.B. $U_{B}$ in Schritten von $20 \mathrm{~V}, I$ in Schritten von 0,1 A), welche Durchmesser überhaupt messbar sind.

3. Man wähle mindestens zwei Spulenströme mit möglichst großen Bereichen für $U_{B}$ und umgekehrt mindestens zwei Beschleunigungsspannungen mit möglichst großen Bereichen für $I$, in denen der Durchmesser messbar ist. Dann wird unter Festhaltung des einen einstellbaren Parameters in Abhängigkeit des zweiten einstellbaren Parameters der Durchmesser bestimmt. Diese Methode ist sinnvoll um später systematische Fehler erkennen zu können. Insgesamt sollten etwa 25 Durchmesser $d=d\left(U_{B}, I\right)$ bestimmt werden.

4. Notieren Sie bitte die Mess- und Ablesefehler sowie die Spulendaten.

\subsection{Angaben}

Spulendaten: Radius $R=12,2 \mathrm{~cm}$; Windungen $n=200$ (pro Spule). 


\subsection{Auswertung}

1. Die einzelnen Berechnungsschritte sollten beschrieben und die Einzelwerte $\left(U, I, d, e / m_{e}\right)$ übersichtlich dargestellt werden.

2. Berechnung der spezifischen Elektronenladung $e / m_{e}$ für alle Wertepaare

3. In der Auswertung sollte auf eine exakte Fehlerrechnung geachtet werden.

4. Für eine Diskussion möglicher systematischer Fehler bietet es sich an, die ermittelten $\frac{e}{m_{e}}$ Werte in Abhängigkeit vom Radius grafisch darzustellen.

5. Für ein Wertepaar nehmen Sie $e / m_{e}$ als gegeben an und berechnen sie die Flußdichte $B$ aus den Messwerten und vergleichen Sie das Ergebniss mit dem theoretisch erwarteten. 


\section{Der Franck-Hertz-Versuch}

... In Wirklichkeit war es eine wichtige Bestätigung der Grundannahmen der neuen Bohrschen Theorie des Atoms. Wir haben das selbst damals noch nicht voll verstanden, es hat sich dann kurz hinterher herausgestellt. ...

Aus einer Rede von Gustav Hertz zum Franck-Hertz-Versuch. James Franck war übrigens der Gründer des II. Physikalischen Institutes in Göttingen.

\subsection{Stichworte}

Franck-Hertz-Versuch, Energiestufen der Atome, elastischer und unelastischer Stoß, Anregung und Ionisation, freie Weglänge, Raumladung, Vakuumdiode, Glühemission.

\subsection{Literatur}

NPP: 44 und 45; Gerthsen; BS-4 und BS-3; Dem-3; Geschke; Wal.

\subsection{Zubehör}

Anstelle der »klassischen« Variante mit Quecksilber wird in diesem Versuch mit einer mit Neon gefüllten Röhre gearbeitet, die zwar ein etwas komplexeres Termschema aufweist, aber experimentell deutlich leichter zu handhaben ist.

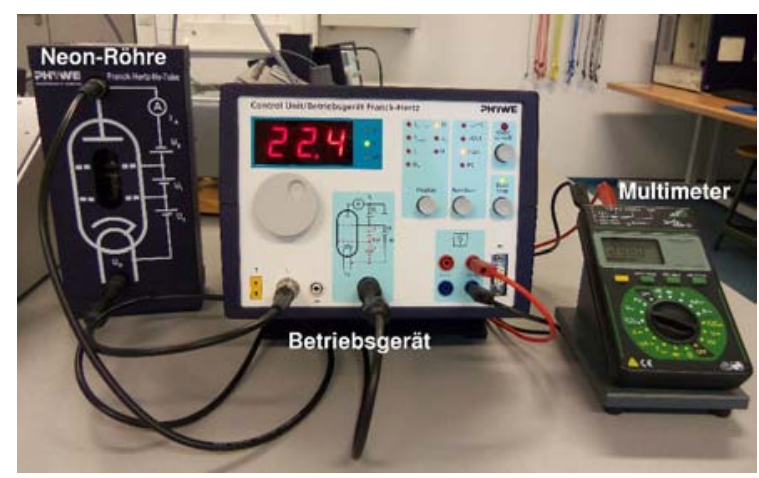

Bild 20.1: Der »Franck-Hertz-Versuch« mit Neon.

Bild 20.1 zeigt ein Foto des Versuches mit Zubehör: Franck-Hertz-Rohr, Betriebsgerät für die Versorgungsspannungen und Multimeter zur Strommessung. 


\subsection{Grundlagen}

Der Franck-Hertz-Versuch bestätigte die Bohrschen Postulate durch den Nachweis der diskreten Energieabgabe von beschleunigten Elektronen an Quecksilberatome in einem triodenartigen Vakuumrohr.

Die Verknüpfung der Anregungsenergie eines Atoms und der Wellenlänge des ausgesandten Lichts bei der Rückkehr in den Grundzustand durch die Bohrsche Frequenzbedingung:

$$
E_{a}=h \cdot v=\frac{h \cdot c}{\lambda}
$$

kann durch Messungen direkt nachgeprüft werden. Das gelang in den Jahren 1911 bis 1914 durch einen Versuchsaufbau von James Franck und Gustav Hertz.

In einer mit Quecksilberdampf (Originalversuch) bzw. in unserem Versuch mit Neon (Ne) gefüllten Röhre werden von einer Glühkathode $\mathbf{K}$ ausgehende Elektronen zunächst vom Raumladungsgitter $\mathbf{G}_{1}$ abgesaugt, so dass weitere Elektronen aus der Kathode austreten können. Die Spannung an $\mathbf{G}_{1}$ regelt damit die Menge der austretenden Elektronen. Durch eine regelbare Beschleunigungsspannung $\mathbf{U}=0-30 \mathrm{~V}$ zwischen der Kathode und dem Gitter $\mathbf{G}_{2}$ werden die durch $\mathbf{G}_{1}$ tretenden Elektronen beschleunigt. Im Raum zwischen Gitter $\mathbf{G}_{2}$ und Kathode $\mathbf{K}$ können sie außerdem durch Anregung der dazwischen liegenden Quecksilberatome Energie verlieren. Hinter dem Gitter befindet sich eine Auffangelektrode A, die mit einer geringen Spannung negativ gegen das Gitter aufgeladen ist, so dass nur die Elektronen sie erreichen können, die genügend Energie haben, um die Bremsspannung zu überwinden. Die Zahl dieser Elektronen wird als Strom (»Auffängerstrom«) gemessen. Als Messergebnis erhält man mit steigender Beschleu-

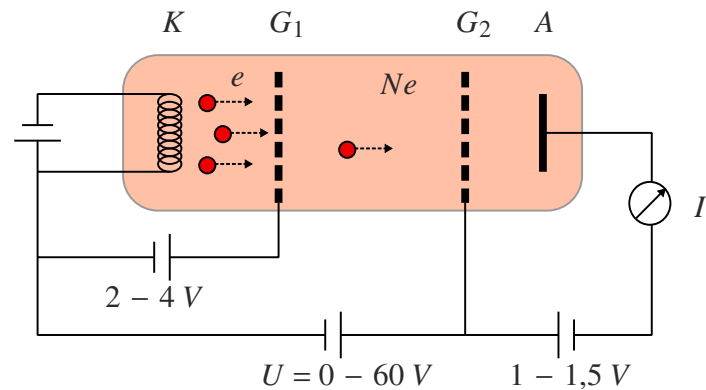

Bild 20.2: Schematischer Aufbau der Franck-Hertz-Röhre: A Auffänger 1 bis $1.5 \mathrm{~V}$ negativ gegen $\mathrm{G}_{2}$, fest eingestellt; $G_{2}$ Beschleunigungsgitter, Spannung regelbar; $\mathrm{G}_{1}$ Raumladungsgitter 2 bis $4 \mathrm{~V}$ positiv gegen $\mathrm{K}$ - fest eingestellte Kathode.

nigungsspannung zunächst einen stark ansteigenden Strom, da die Elektronen keine Energie verlieren können, weil ihre Gesamtenergie nicht zur Anregung des ersten unbesetzten Niveaus ausreicht. Bei einer bestimmten Spannung, die vom Material zwischen Gitter $\mathbf{G}_{2}$ und Kathode $\mathbf{K}$ abhängt, sinkt der Strom plötzlich ab, die Elektronen erhalten genug Energie, um das erste Niveau anzuregen und können, nachdem sie das getan haben, die Auffangelektrode nicht erreichen. Erhöht man die Spannung weiter, so steigt der Strom zunächst wieder an, um bei der doppelten Spannung, wenn zwei anregende Stöße möglich werden, erneut abzusinken. An den Stellen des starken Stromabfalls ist also

$$
e \cdot U=h \cdot v
$$


wenn $e$ die Elementarladung, $U$ die Beschleunigungsspanung, $h$ die Plancksche Konstante und $v$ die Frequenz des Lichtes ist, das bei der Rückkehr in den Grundzustand ausgestrahlt wird. Man erhält also aus diesen Messungen einen direkten Zusammenhang zwischen elektrisch gemessener Energie und Lichtwellenlängen. FrancK und HerTz fanden beim Quecksilber eine Anregungsenergie von 4,9 eV und eine aus gesandte Wellenlänge von 253,7 nm. Bei Neon wird eine Anregungsenergie von $18,3 \mathrm{eV}$ bzw. $18,9 \mathrm{eV}$ bei ausgesandten Wellenlängen im roten Bereich beobachtet.

\subsection{Fragen}

1. Welche Aussagen macht das BoHrsche Atommodell und wie können diese durch den FranckHertz-Versuch bestätigt werden?

- Wovon ist die Energie eines Elektrons abhängig?

- Welche Energiezustände können die $\mathrm{e}^{-}$annehmen?

2. Was bezeichnet man als mittlere freie Weglänge?

3. Was ist Anregung? Wann spricht man von Ionisation?

4. Skizzieren Sie den erwarteten Zusammenhang zwischen $I$ und $U$. Wie wäre der Kurvenverlauf, wenn die Röhre nicht mit einem Gas gefüllt wäre? Erklären Sie die Unterschiede?

- Wodurch ist die Spannungsdifferenz von knapp 19 V zwischen zwei Maxima zu erklären?

- Was sind elastische und inelastische Stöße?

- Bei welchen Stößen tritt eine Anregung auf ein nächsthöheres Energieniveau auf?

- Worauf deutet ein Stromabfall hin?

- Weshalb fällt der Auffängerstrom beim Erreichen der Minima nicht ganz auf Null zurück?

5. Wie ist der Franck-Hertz-Versuch aufgebaut? Erläutern Sie die Funktion der einzelnen Bauteile.

\subsection{Hinweise}

1. Die Ne-Röhre muss nicht beheizt werden und ist daher jederzeit betriebsbereit. Entsprechend werden Anschlüsse und Bedienelemente des Betriebsgeräts, die den Ofen betreffen, nicht verwendet.

2. Die Notation der Betriebsspannungen und die Beschaltung der Beschleunigungsspannung weichen von Abb. 20.2 ab und sollten dem Schaltplan auf dem Betriebsgerät entnommen werden.

3. Ein zu großer Elektronenstrom kann zu einer unerwünschten Gasentladung führen, der die Röhre potentiell beschädigen kann. Deshalb darf das Franck-Hertz-Rohr nur mit sehr kleinen Stromstärken am Auffänger in der Größenordnung einiger $10^{-9} \mathrm{~A}$ betrieben werden. Das Betriebsgerät sollte selbsttätig vor Gasentladungen schützen, jedoch sollte bei einem sehr starken Stromanstieg die Beschleunigungsspannung sofort verringert werden. 


\subsection{Durchführung}

1. Betriebsgerät mit der Ne-Röhre und Messgerät verbinden: DIN-Buchse am Betriebsgerät unter der Schaltskizze (unten, 4. Buchse von links) mit der unteren Buchse der Röhre unter $U_{\mathrm{H}}$ und BNC-Buchse $» I_{\mathrm{A}}$ « am Betriebsgerät (unten, 2. Buchse von links) mit der oberen Buchse der Röhre verbinden. Ein Spannungsmessgerät mit den Ausgängen $U \sim I_{\mathrm{A}}$ des Betriebsgeräts verbinden; dieses zeigt eine Spannung proportional zum Auffängerstrom $I_{\mathrm{A}}$ der Röhre an, wobei die Nullage verschoben ist und im deaktivierten Zustand (LED »Start/Stop« ist aus) notiert werden sollte.

2. Betriebsgerät einschalten; die LED »Start/Stop« sollte aus sein (sonst Knopf darunter betätigen). Mit dem »Function«-Knopf die LED auf »man.«stellen und mit dem »Display«Knopf $U_{\mathrm{H}}$ bzw. $U_{2}$ oder $U_{3}$ auswählen und die entsprechende Spannung wie am Aufbau angegeben mit dem Drehknopf unter dem Display einstellen. $U_{1}$ mit dem »Display «-Knopf auswählen und auf 0 stellen. »Start/Stop«-LED per »Function«Knopf aktivieren.

3. Messung des Auffängerstroms als Funktion der Beschleunigungsspannung $U_{1}$ in Schritten von $0,5 \mathrm{~V}$ von 0 bis $90 \mathrm{~V}$ bzw. bis zum Erreichen des max. messbaren Stroms. In der Nähe der Maxima empfiehlt es sich ggf. in keineren Schritten von $U_{1}$ zu messen. $U_{1}$ kann vom Display des Betriebsgeräts, $I_{\mathrm{A}}$ vom Spannungsmessgerät abgelesen werden.

4. Beobachten Sie während der Messung des Auffängerstroms die Leutcherscheinungen in der Röhre und notieren diese. Dazu sollte der Raum abgedunkelt sein.

\subsection{Auswertung}

1. Grafische Darstellung der Messdaten schon während der Messung.

2. Bestimmung der Maxima aus den Schnittpunkten der Tangenten an die Linienflanken. (Welchen Vorteil hat dieses Verfahren?)

3. Bestimmung der Anregungsenergie des Quecksilbers und daraus die Wellenlänge des optischen Übergangs zwischen dem angeregten Niveau und dem Grundniveau des Atoms.

4. Welchem Übergang entspricht dies? Skizzieren Sie bitte das Energieschema. Nur bei der Ne-Röhre: vergleichen Sie die Leuchterscheinungen mit den Strom-Spannungsmessdaten. Warum leuchtet das Gas im roten Wellenlängenbereich? 


\section{Balmerserie von Wasserstoff}

Das Wasserstoffatom gehört zu den Systemen, deren Verhalten über die Schrödingergleichung des Elektrons im Coulombpotenzial des Kerns quantenmechanisch vollständig analytisch berechnet werden kann. Die diskreten Energieniveaus führen zu einem Emissionsspektrum, was zunächst empirisch von BALMER beschrieben wurde und nachfolgend erstmalig durch das Bohrsche Atommodell erklärt werden konnte.

\subsection{Stichworte}

Wasserstoffatom, Balmerserie, Quantenstruktur der Atome, diskrete Energieniveaus, RydbergKonstante.

\subsection{Literatur}

Dem-3; Gerthsen; BS-3 und BS-4.

\subsection{Zubehör}

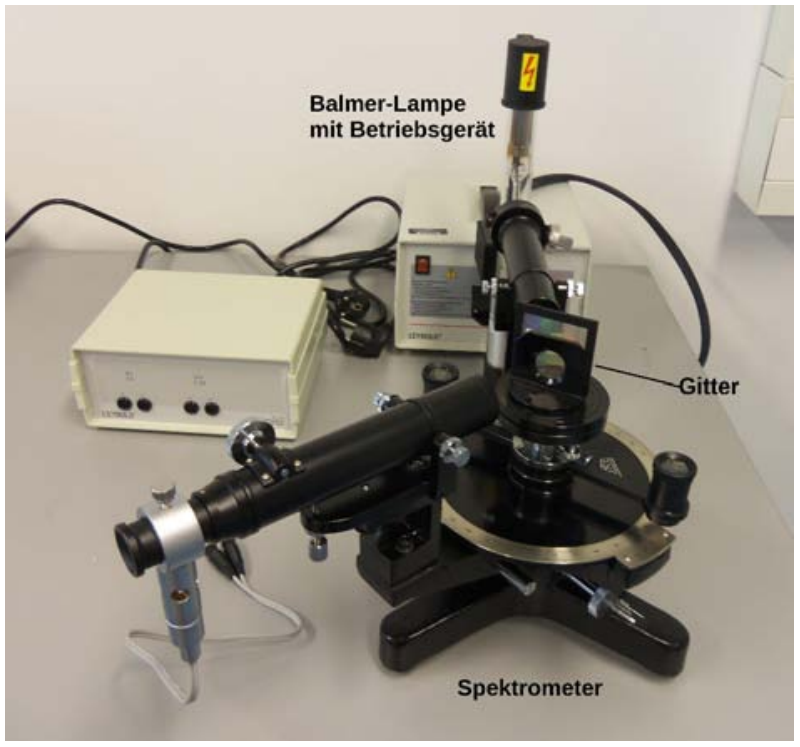

Bild 21.1: Der Versuch »Balmerserie von Wasserstoff «

Bild 21.1 zeigt ein Foto des Versuchsaufbaus mit Zubehör: Spektrometer mit Gitter wie in Kapitel 16 beschrieben und Balmer-Lampe mit Betriebsgerät. Die Hg-Cd-Lampe zur Kalibration 
des Spektrometers ist im Bild nicht gezeigt und identisch zu der in Bild 16.1. Im Gegensatz zum vorherigen Versuch stehen hier die optischen Eigenschaften des Spektrometer im Hintergrund. Stattdessen wird es als spektroskopisches Messgerät eingesetzt, um atomare Energieniveaus zu bestimmen.

\subsection{Grundlagen}

Als Balmerserie wird eine Abfolge von Spektrallinien der vom Wasserstoffatom emittierten Strahlung bezeichnet, die zunächst empirisch von JoHANN JAKов BALMER 1885 beschrieben wurde, was später durch JoHANNES RYDBERG mit folgender Gleichung verallgemeinert wurde:

$$
\lambda^{-1}=R_{\infty} \cdot\left(\frac{1}{4}-\frac{1}{n^{2}}\right)
$$

mit der Rydberg-Konstante $R_{\infty}$ und einer ganzen Zahl $n>2$.

Die quantenmechanische Berechnung der Energieniveaus im Wasserstoffatom verwendet die Schrödingergleichung für die Wellenfunktion $\psi(r, \vartheta, \phi)$ des Elektrons mit Energie $E$ im Coulombpotenzial $V(r)$ des Kerns in Kugelkoordinaten:

$$
\left(-\frac{\hbar^{2}}{2 m_{e}} \Delta+V(r)\right) \psi(r, \vartheta, \phi)=E \cdot \psi(r, \vartheta, \phi) .
$$

Wird der Laplace-Operator $\Delta$ ebenfalls in Kugelkoordinaten ausgedrückt, so erlaubt die Variablentrennung $\psi(r, \vartheta, \phi)=R(r) \cdot \Theta(\vartheta) \cdot \Phi(\phi)$ die separate Berechnung der Radialwellenfunktion $R(r)$ und des nur durch die Drehimpulsquantenzahlen bestimmten winkelabhängigen Teils $\Theta(\vartheta) \cdot \Phi(\phi)$. Die $1 / r$-Abhängigkeit des Coulombpotenzials bedingt, dass die Energieniveaus $E_{n}$ in den Drehimpulsquantenzahlen entartet sind, d.h. nur von der Hauptquantenzahl $n$ abhängen:

$$
E_{n}=\frac{\alpha^{2} m_{e} c^{2}}{2} \cdot \frac{1}{n^{2}}
$$

mit der Feinstrukturkonstante $\alpha$, der Elektronenmasse $m_{e}$, dem Planckschen Wirkungsquantum $h$ und der Lichtgeschwindigkeit $c$. Damit kann man das Spektrum der Balmer-Formel aus G1. (21.1) als Abstrahlung beim Übergang von einem Energieniveau mit $n>2$ zu einem Niveau mit $n=2$ deuten. Die Rydberg-Konstante wird damit zu

$$
R_{\infty}=\frac{\alpha^{2} m_{e} c}{2 h}
$$

Die Rydberg-Konstante lässt sich also aus anderen Naturkonstanten berechnen. Durch die mittlerweile sehr präzise Vermessung von Atomspektren ist die Rydberg-Konstante die am genauesten bestimmte Naturkonstante. 


\subsection{Fragen}

1. Wodurch ist die Wellenlänge einer Balmerlinie nach dem quantenmechanischen Modell des Wasserstoffatoms definiert?

2. Auf welches Energie-Grundniveau des Wasserstoffatoms bezieht sich die Balmerserie? Was beobachtet man bei den anderen Grundniveaus?

3. Warum genügt es bei der Berechnung der Quantenzustände des Wasserstoffatoms, den Atomkern näherungsweise durch ein ruhendes Coulombpotenzial darzustellen? Welche Korrektur müsste man entsprechend an der Rydberg-Konstante vornehmen, die genau genommen nur für einen unendlich schweren Kern gilt?

\subsection{Durchführung}

In diesem Versuch wird das Gitterspektrometer aus Versuch 16 verwendet: Dieses wird daher zunächst mit der Quecksilber-Cadmium-Lampe wie in Abschnitt 16.6.2 beschrieben justiert.

1. Vermessen Sie die in Tabelle 16.1 angegebenen Linien der Hg-Cd-Lampe und notieren Sie die Ablenkungswinkel $\alpha$ für die 1. Ordnung.

2. Tauschen Sie nun die Hg-Cd-Lampe gegen die Balmer-Lampe und vermessen alle sichtbaren Linien für die 1. Ordnung.

\subsection{Auswertung}

1. Kalibrieren Sie das Spektrometer aus Messung 1, indem Sie die Wellenlängen der Hg-CdLampe als bekannt voraussetzen und durch Auftragen von $\sin \alpha$ gegen die Wellenlängen und einer lineare Regression die Gitterkonstante bestimmen.

2. Berechnen Sie mit dieser Kalibration die in Messung 2 beobachteten Wellenlängen. Identifizieren Sie diese mit den aus der Balmer-Formel Gl. (21.1) zu erwartenden Energieniveaus und berechnen so die Rydberg-Konstante. 


\section{Röntgenstrahlung}

Röntgenstrahlung wird heutzutage in unterschiedlichsten Anwendungsgebieten genutzt, die in der medizinischen Diagnostik verwendeten Geräte sind wohl die bekanntesten Beispiele. Doch auch in der Analyse von Kristallqualität und -struktur sowie zur Phasenanalyse sind sie zur Standardmethode geworden, da die Gitterkonstanten fester Körper $(0,1 \mathrm{~nm})$ gerade in ihrem Wellenlängenbereich (100 - 0,01 nm) liegen und man daher optisch die Gitterstrukturen charakterisieren kann (BRAGG-Reflexion).

\subsection{Stichworte}

Aufbau einer Röntgenröhre, charakteristische Strahlung, Bremsstrahlung, Absorption von Röntgenstrahlung in Materie, Absorptionskanten, Kristallspektrometer, Bragg-Reflexion, Geiger-Müller-Zählrohr, Totzeit.

\subsection{Literatur}

Pohl; Grimsehl; Gerthsen; BS-6: ; Schpolski: Atomphysik I; Dem-3; K. Hermbecker: Handbook of Physics X-ray experiments, Phywe [29].

\subsection{Zubehör}

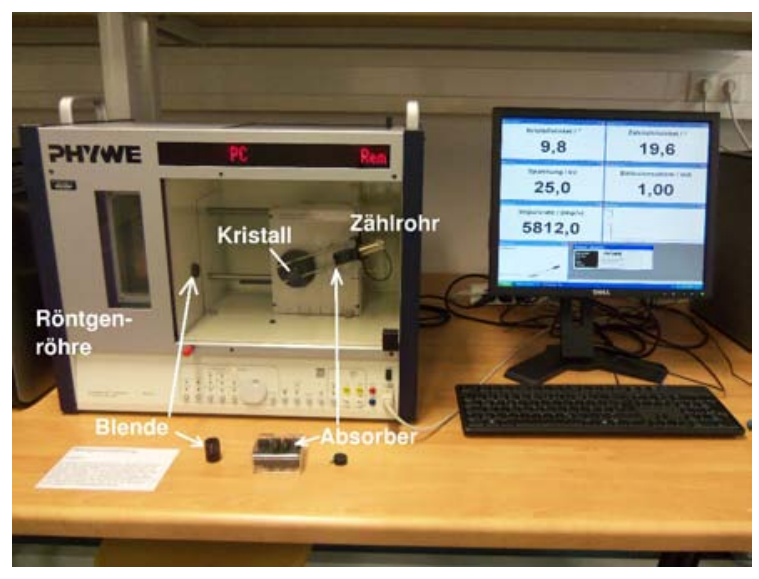

Bild 22.1: Der Versuch »Röntgenstrahlung «.

Bild 22.1zeigt ein Foto des Versuches mit Zubehör. Es stehen 3 verschiedene Geräte zur Verfügung, die bis auf das Anodenmaterial der Röntgenröhre identisch sind ( $\mathrm{Cu}, \mathrm{Mo}, \mathrm{Fe})$. Als Anodenmaterialien werden im Versuch Kupfer, Eisen und Molybdän verwendet. Die Beugungskristalle 
sind fest in den Apparaturen installiert: LiF (201 pm), KBr (329 pm). Die weiteren Zubehörteile sind: Halogenzählrohr (Geiger-Müller-Zählrohr), Abschirmung, Blenden mit Durchmessern von 1, 2 und $5 \mathrm{~mm}$, Monochromatoren ( $\mathrm{Zr}$ für $\mathrm{Mo}-\mathrm{K} \alpha$, Ni für $\mathrm{Cu}-\mathrm{K} \alpha$ ), Al, Zn, Cu, Ni und Sn Absorberfolien mit unterschiedlichen Dicken.

\subsection{Grundlagen}

\subsubsection{Röntgenspektrometer}

Die Röntgenröhre besteht im Wesentlichen aus einer Glühkathode und einer abgeschrägten Anode, die beide in einem evakuierten Kolben angebracht sind, s. Abb. 22.2. Durch die zwischen Kathode und Anode anliegende Beschleunigungsspannung (im Versuch bis $35 \mathrm{kV}$, bei diversen Anwendungen häufig auch bis in den MV Bereich) werden Elektronen zur Anode hin beschleunigt und erzeugen beim Auftreffen Bremsstrahlung und Charakteristische Strahlung. Daneben wird der größte Teil der Elektronenenergie in Wärme umgesetzt. Die Anode einer Röntgenröhre muss also immer gut gekühlt werden, da sie ansonsten zerstört werden würde.

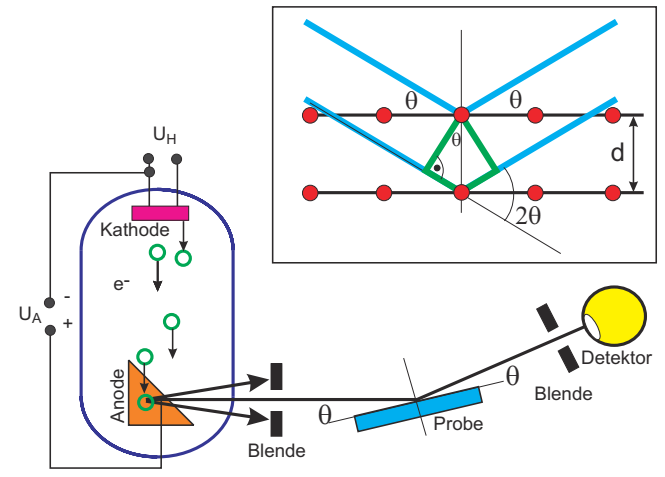

Bild 22.2: Schematischer Aufbau eines Röntgenspektrometers mit Röntgenröhre, Kristall und Detektor. Im oberen Detail ist die Reflexion und Interferenz der Röntgenstrahlung an den Netzebenen des Kristalls (Bragg-Reflexion) verdeutlicht.

Zur Analyse des Energiespektrums der Röntgenstrahlung wird im Versuch die Bragg-Reflexion an einem Kristall (LiF oder KBr) verwendet. Dabei werden die Photonen an den einzelnen Atomen des Kristalls gestreut, was im Wellenbild als Reflexion an den Netzebenen gedeutet werden kann. Die auslaufenden Wellen interferieren nun miteinander, wobei die Winkellage der Maxima von der Photonenenergie abhängig ist. Mit Hilfe der Bragg-Bedingung

$$
2 d \sin \theta=n \lambda
$$

lässt sich nun über die Messung der Winkelverteilung der Intensität $I(\theta)$ auf die Energie- bzw. Wellenlängenverteilung der Intensität $I(\lambda)$ zurück schließen. Die Werte für $d$ sind für die verwendeten Kristalle sehr gut bekannt (s.u.).

\subsubsection{Geiger-Müller-Zählrohr}

Um die Intensität des Photonenstrahls zu messen wird ein Geiger-Müller-Zählrohr verwendet, das in ähnlicher Form auch für den Versuch »Radioaktivität« eingesetzt wird. 
Das Zählrohr ist ein Ionisationsgerät, das auf einer Verstärkung schwacher, primärer Ionisationsprozesse beruht. Es besteht aus einem zylindrischen Kondensator, s. Abb. 22.3 Die zentrale Elektrode dient als Anode und besteht aus einem Wolfram-Draht mit einem sehr kleinen Radius (Größenordnung $\mu \mathrm{m}$ ). Die Kathode ist ein metallischer Zylinder. Als Betriebsgas wird in der Praktikumsanordnung ein Halogen verwandt. Wegen der zylindrischen Anordnung treten in der Nähe des Drahtes sehr hohe Feldstärken auf, so dass durch Ionisation freigesetzte Elektronen durch Stoßionisation neue Elektronen und Ionen erzeugen können. Hierauf beruht der Verstärkungseffekt.

Je nach Betriebsspannung unterscheidet man verschiedene Gas-Detektoren, die unterschiedliche Zwecke erfüllen. Die unterschiedlichen Spannungsbereiche führen zu unterschiedlichen Gasverstärkungen und so zu Ionisationskammer, Proportionalzählrohr und Auslösezähler (GeigerMüller-Zählrohr). Eine wichtige Kenngröße für Zählrohre ist die so genannte Totzeit, d.h. die Zeit, die nach einem Signal vergeht, bis das nächste Signal aufgenommen werden kann. Insbesondere bei hohen Zählraten führt die Totzeit $\tau$ zu einer deutlichen Verringerung der gezählten Ereignisse, man kann diesen Fehler durch die folgende Formel korrigieren:

$$
N_{\text {korrigiert }}=\frac{N_{\text {gemessen }}}{1-\tau \cdot N_{\text {gemessen }}}
$$

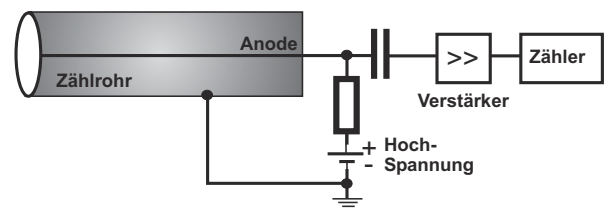

Bild 22.3: Prinzipskizze eines Geiger-Müller-

Zählrohres. Durch die zylindrische Anordnung der Elektroden entstehen in der Nähe der zentralen Anode sehr hohe elektrische Feldstärken, die eine StoBionisation des Füllgases ermöglichen.

\subsubsection{Charakteristische Röntgenstrahlung}

Das Bohrsche Atommodell beschreibt die Atome mit Elektronen, die auf bestimmten Bahnen um den Kern mit Ladung +Ze umlaufen. Diese Bahnen können nur bestimmte Quantenzahlen annehmen (Hauptquantenzahl $n$, Nebenquantenzahl $l$, Magnetische Quantenzahl $m$ und Spin $s$ ). Aus diesen Quantenzahlen kann man die Energie der jeweiligen Zustände berechnen. Kennt man die Energieniveaus der Atome des Anodenmaterials, kann man die Energie der charakteristischen Röntgenstrahlung leicht berechnen, da diese erzeugt (oder absorbiert) wird durch den Übergang eines Elektrons von einem auf einen anderen Zustand:

$$
E_{\mathrm{ph}}=h v=E_{s}-E_{f}
$$

Für die $\mathrm{K} \alpha_{1}$ - und $\mathrm{K} \alpha_{2}$-Linie ist dies zum Beispiel der Übergang eines Elektrons von der L-Schale $(n=2)$ auf die K-Schale $(n=1)$. Die Existenz der eng benachbarten $\mathrm{K} \alpha_{1}$ - und $\mathrm{K} \alpha_{2}$-Linien beruht auf der Feinstruktur des Atombaus. Die L-Schale ist durch die Feinstruktur in die Unterschalen $\mathrm{L}_{1}, \mathrm{~L}_{2}$ und $L_{3}$ energetisch aufgespalten 1

$1 \mathrm{~K} \alpha_{1}: \mathrm{L}_{3} \rightarrow K$ und $\mathrm{K} \alpha_{2}: \mathrm{L}_{2} \rightarrow K . \mathrm{K} \beta: \mathrm{M} \rightarrow K$. 
Analog zu den Serienspektren in der Atomphysik (Balmer-Serie, siehe Versuch21) kann auch die charakteristische Röntgenstrahlung für Material der Kernladungszahl $Z$ durch ein analoges Gesetz, das Moseleysche-Gesetz, beschrieben werden:

$$
v_{\mathrm{K}}=R_{v}(Z-1)^{2}\left(\frac{1}{n_{f}^{2}}-\frac{1}{n_{s}^{2}}\right)
$$

Für K-Linien muss gelten $n_{f}=1$. Die Konstante $R_{v}$ ist auch hier die Rydberg-Konstante mit $R_{v}=$ $3,2898 \cdot 10^{15} \mathrm{~Hz}$. Die anziehende Kernladungszahl wir um die Zahl 1 verringert, da dass zweite Elektron auf der K-Schale die Kernladung abschirmt. Eine etwas allgemeineres MoselEysches Gesetz kann man auch für die L-Schale schreiben:

$$
\nu_{\mathrm{L}}=R_{v}\left(Z-\sigma_{\mathrm{L}}\right)^{2}\left(\frac{1}{n_{f}^{2}}-\frac{1}{n_{s}^{2}}\right)
$$

Die Abschirmung für die L-Schale ist $\sigma_{\mathrm{L}}=7,4$, was bedeutet, dass sich in der L-Schale etwa 7 bis 8 Elektronen befinden sollten, gegenüber 2 Elektronen in der K-Schale $\left(\sigma_{K}=1\right)$. Hiermit können die charakteristischen Linien der Röntgenstrahlung gut erklärt werden.

\subsubsection{Röntgenstrahlung in Abhängigkeit der Anodenspannung}

Als Funktion der Anodenspannung $U_{A}$ ändern sich einige Parameter des Röntgenspektrums. Einerseits wird bei Vergrößerung von $U_{A}$ die Grenzwellenlänge $\lambda_{\mathrm{gr}}$ des Bremskontinuums hin zu höheren Energien verschoben. Dies wurde auch als DuANE-HunT-Verschiebung bekann 2 , in der Originalversion, in der es 1915 experimentell entdeckt wurde:

$$
\lambda_{\mathrm{gr}} \cdot U_{A}=\text { const. }=\frac{h \cdot c}{e}=1,234 \cdot 10^{-6} \mathrm{~V} \mathrm{~m}
$$

Heute wissen wir, dass dies mit der Energieerhaltung

$$
\frac{h c}{\lambda_{\mathrm{gr}}}=e \cdot U_{A}
$$

zusammenhängt. Das bedeutet, die Berechnung der Grenzwellenlänge geht von der Idealisierung aus, dass ein Elektron seine ganze kinetische Energie in ein einziges Röntgenquant abgibt.

Andererseits ändert sich aber auch die Intensität der Röntgenstrahlung. Die Intensität der charakteristischen Strahlung $I_{K}$ ändert sich mit der Anodenspannung $U_{A}$, dem Anodenstrom $I_{A}$ und dem Ionisationspotenzial der entsprechenden Schale $U_{K}$ wie

$$
I_{K} \sim I_{A} \cdot\left(U_{A}-U_{K}\right)^{3 / 2} .
$$

2 In Anlehnung an das WIENsche Verschiebungsgesetz. 


\subsubsection{Absorption von Röntgenstrahlung}

Die Intensität von Photonenstrahlen kann im Wesentlichen auf drei unterschiedliche Arten gemindert werden, d.h. es gibt drei verschiedene Wechselwirkungsmechanismen:

- Photoeffekt

- Comptonstreuung

- Paarproduktion

Für den Energiebereich der Röntgenstrahlung dominiert die Absorption durch den Photoeffekt. Um dies zu untersuchen, werden verschiedene Folien in den Röntgenstrahl gegeben und die Energieverteilung der Intensität mit und ohne Folie gemessen. Die Absorption hängt dabei einserseits von der Dicke der Folien ab (Gesetz von Lambert), andererseits aber auch von der Schalenstruktur des spezifischen Materials (Moseley-Gesetz). Letzterer Effekt führt zu den Absorptionskanten, die als sprunghafte Änderung - beispielsweise des Wirkungsquerschnitts in Bild 22.4- sichtbar sind.

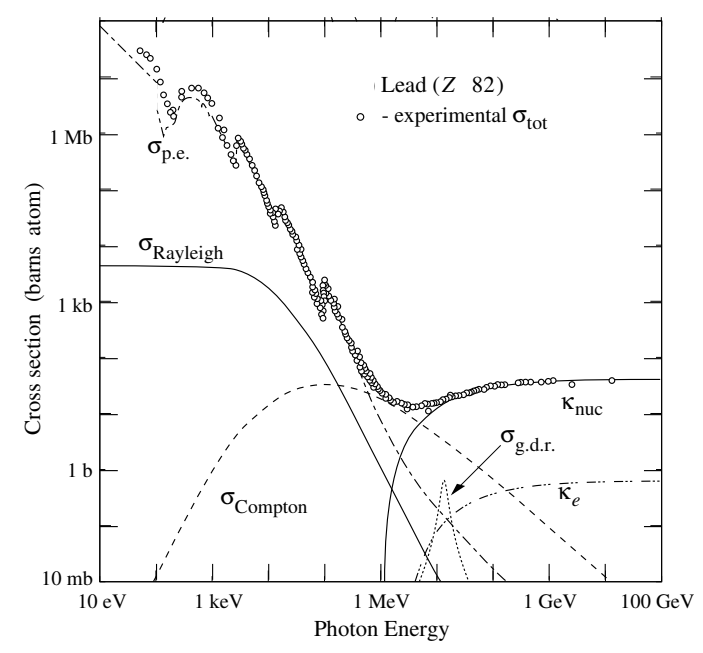

Bild 22.4: Zusammensetzung des totalen Wirkungsquerschnitts $\sigma_{\text {tot }}$ für die Wechselwirkungen zwischen Photonen und Blei. $\sigma_{\text {p.e. }}$ : Photoeffekt; $\sigma_{\text {Rayleigh }}$ : Rayleighstreuung; $\sigma_{\text {Compton }}$ : Comptonstreuung; $\kappa_{\text {nuc }}$ : Paarproduktion im Kernfeld; $\kappa_{e}$ : Paarproduktion im Elektronenfeld; $\sigma_{\text {nuc }}$ : Absorption des Photons vom Kern; $\sigma_{\text {g.d.r. }}$ : Photon-KernWechselwirkung, hauptsächlich "giant dipole resonance" (Quelle: http://pdg.lbl.gov/).

\subsection{Fragen}

1. Wie entsteht Röntgenbremsstrahlung? Skizzieren Sie eine typische Energieverteilung der Intensität! Wie kann man die Form der Verteilung erklären?

2. Wie entsteht charakteristische Röntgenstrahlung? Skizzieren Sie eine typische Energieverteilung der Intensität! Wie kann man die Form der Verteilung erklären?

3. Was kann man aus der kurzwelligen Grenze des Bremsspektrums ablesen?

4. Wie groß ist die Ausbeute an Röntgenstrahlung? Was geschieht mit der restlichen Energie?

5. Warum ist der Anodenblock um $45^{\circ}$ geneigt?

6. Erklären Sie die Funktionsweise eines Kristallspektrometers. Warum misst man beim Spektrometer einen Winkel von $2 \theta$ ? 
7. Zeichnen Sie den typischen Verlauf der Impulsrate eines Zählrohres als Funktion der Zählrohrspannung. Erklären Sie in diesem Zusammenhang die Begriffe Ionisationsbereich, Proportionalitätsbereich, Auslösebereich.

8. Welcher Bereich wird im Versuch verwendet?

9. Wieso muss die Paarproduktion bei der Absorption der Röntgenstrahlung in diesem Versuch nicht berücksichtigt werden?

10. Wie lautet die Moseley-Formel für die Energien der charakteristischen Strahlung? Was kann man also über die Absorptionsenergie von Atomen mit großem $Z$ sagen?

\subsection{Durchführung}

Vorbemerkung: Das im Experiment verwendete Röntgengerät ist durch Bleiglas gesichert (Vollschutzgerät). Wird die Fronttür geöffnet (Drücken und Drehen des roten Sicherheitsschalters an der Frontseite des Gerätes), schaltet sich die Röhre automatisch aus. Allerdings sollte man sich beim Betrieb des Geräts nicht unnötig lange in dessen unmittelbarer Nähe $(<10 \mathrm{~cm}$ Abstand) aufhalten. Die Röntgenröhre sollte in den ersten 10 Minuten mit maximal 25 kV Anodenspannung betrieben werden.

Die Steuerung des Gerätes und die Messwertaufnahme wird durch den daneben stehenden Computer (login: Prakt ohne Password) und das Programm »measure « (Desktop-Symbol) übernommen. Hier können alle relevanten Parameter, wie Schrittweite, Messzeit, Winkel etc. eingestellt und die Messung durchgeführt werden. Die Daten können abgespeichert werden. Legen Sie sich hierzu ein geeignetes Verzeichnis an und ändern Sie bitte nichts in anderen Verzeichnissen. Die Auswertung der Spektren kann auch mit diesem Programm, das auf der Praktikumswebseite erhältlich ist, durchgeführt werden. Wird ein anderes Programm verwendet, müssen die Spektren als ASCII-Files gesichert werden. Nach Beendigung des Versuches übertragen Sie Ihre Daten bitte per Internet auf ein Ihnen zugänglichen Rechner oder sichern die Dateien auf einem USB-Stick.

1. Das Anodenmaterial der Apparatur ist zu notieren (steht auf der linken Seitenwand des Gerätes). Die $2 \mathrm{~mm}$ Blende ist bereits eingesetzt 3

2. Untersuchung der charakteristischen Strahlung der Röhre. Dazu wird ein Spektrum mit $\mathrm{U}=25 \mathrm{kV}, \mathrm{I}=1 \mathrm{~mA}, \Delta \theta=0.1^{\circ}$ und $\Delta \mathrm{t}=2 \mathrm{~s}$ aufgenommen 4 Für die unterschiedlichen Anodenmaterialien sind die Winkelbereiche bei gleichem Kristall verschieden und in Tabelle 22.1 angegeben:

3. Untersuchung der Abhängigkeit der Grenzwellenlänge und der Intensität der charakteristischen Strahlung von der Anodenspannung. Bei den Messungen sind $\mathrm{I}=1 \mathrm{~mA}, \Delta \theta=0.1^{\circ}$,

3 Alles benötigte Zubehör findet sich in der Ablage auf dem Gerät, einfach Schiebedeckel öffnen (altes Modell) bzw. in der Schublade unter dem Gerät (neues Modell).

4 Überprüfen Sie bitte bei der ersten Messung, ob die stärkste Linie in ihrer Position mit dem Literaturwert übereinstimmt. Es kann vorkommen, dass jemand unbeabsichtigt das Goniometer des Gerätes dejustiert hat (unbeabsichtigtes Anstoßen, etc.). Das ergibt eine Verschiebung der charakteristischen Linien. Falls dies der Fall ist, geben Sie bitte sofort der Praktikumstechnik/-leitung Bescheid, damit das Gerät wieder justieren werden kann. 
Tabelle 22.1: Parameter für die Aufnahme der charakteristischen Strahlung.

\begin{tabular}{lrrr}
\hline Anode & $\theta_{\min }\left[^{\circ}\right]$ & $\theta_{\max }\left[^{\circ}\right]$ & Kristall \\
\hline $\mathrm{Cu}$ & 3 & 60 & $\mathrm{LiF}$ \\
$\mathrm{Fe}$ & 3 & 80 & $\mathrm{LiF}$ \\
$\mathrm{Mo}$ & 3 & 50 & $\mathrm{LiF}$ \\
\hline
\end{tabular}

$\Delta \mathrm{t}=2 \mathrm{~s}$ (für Mo: 4s). Die Winkelbereiche sind für die unterschiedlichen Röhren verschieden. Messen Sie nun mit den in Tabelle 22.2 dargestellten Bereichen. Das Aufnahmeprogramm bietet die Möglichkeit, automatisch mehrere Messungen mit verschiedenen Parametern durchzuführen. Diese kann, muss aber nicht genutzt werden.

Tabelle 22.2: Parameter für die Messung der Grenzwellenlänge (M[3].

\begin{tabular}{|l|r|r|r|r|l|}
\hline Anode & $\theta_{\min , 1}$ & $\theta_{\max , 1}$ & $\theta_{\min , 2}$ & $\theta_{\max , 2}$ & $\mathrm{U}_{A}[\mathrm{kV}]$ \\
\hline $\mathrm{Cu}$ & $3^{\circ}$ & $15^{\circ}$ & $17^{\circ}$ & $24^{\circ}$ & $35,32,29,26,23$ \\
\hline $\mathrm{Fe}$ & $3^{\circ}$ & $15^{\circ}$ & $24^{\circ}$ & $31^{\circ}$ & $35,32,29,26,23$ \\
\hline $\mathrm{Mo}$ & $3^{\circ}$ & $15^{\circ}$ & - & - & $35,32,29,26,23$ \\
\hline
\end{tabular}

4. Untersuchung der Absorptionskanten von $\mathrm{Ni}$ und $\mathrm{Cu}$ für die Eisen- und Molybdän-Röhren, und von Sn und Zn für die Kupfer-Röhre (Dicke je $0.025 \mathrm{~mm}$ ). Dazu werden die in Tabelle 22.3 aufgeführten Messungen mit $\mathrm{U}=25 \mathrm{kV}, \mathrm{I}=1 \mathrm{~mA}, \Delta \theta=0.1^{\circ}, \Delta \mathrm{t}=30 \mathrm{~s}$ durchgeführt.

Tabelle 22.3: Parameter der Messungen zu Absorptionskanten.

\begin{tabular}{lcccc}
\hline Anode & $\theta_{\min }\left[^{\circ}\right]$ & $\theta_{\max }\left[^{\circ}\right]$ & $\theta_{\min }\left[^{\circ}\right]$ & $\theta_{\max }\left[^{\circ}\right]$ \\
\hline \multirow{3}{*}{ Sn-Absorber } & \multicolumn{2}{c}{ Zn-Absorber } \\
$\mathrm{Cu}$ & \multicolumn{2}{c}{5.5} & 7.5 & \multicolumn{2}{c}{18.0} & 20.0 \\
\hline \multicolumn{3}{c}{ Ni-Absorber } & \multicolumn{2}{c}{ Cu-Absorber } \\
$\mathrm{Fe}$ & 21.0 & 23.0 & 19.5 & 21.5 \\
\hline $\mathrm{Mo}$ & 21.0 & 23.0 & 19.5 & 21.5 \\
\hline
\end{tabular}

5. Untersuchung der Absorption von Materialien mit unterschiedlichem Z. Dazu werden im Bereich $8^{\circ} \leq \theta \leq 16^{\circ}$ mit $\mathrm{U}=25 \mathrm{kV}, \mathrm{I}=1 \mathrm{~mA}, \Delta \theta=1^{\circ}$ und $\Delta \mathrm{t}=30 \mathrm{~s}$ je ein Spektrum ohne Absorber, mit $0.08 \mathrm{~mm} \mathrm{Al,} 0.025 \mathrm{~mm} \mathrm{Zn,} 0.025 \mathrm{~mm}$ Sn und $0.025 \mathrm{~mm} \mathrm{Ni}$ aufgenommen.

\subsection{Angaben}

- Gitterkonstanten: $\mathrm{d}_{\mathrm{LiF}}=201 \mathrm{pm} ; \mathrm{d}_{\mathrm{KBr}}=329 \mathrm{pm}$. 
- Dichten: $\rho_{S n}=7.28 \mathrm{~g} / \mathrm{cm}^{3} ; \rho_{Z n}=7.14 \mathrm{~g} / \mathrm{cm}^{3} ; \rho_{N i}=8.90 \mathrm{~g} / \mathrm{cm}^{3} ; \rho_{C u}=8.92 \mathrm{~g} / \mathrm{cm}^{3} ; \rho_{A l}=2.7$ $\mathrm{g} / \mathrm{cm}^{3}$.

- Rydberg-Konstante: $R_{v}=3,2899 \cdot 10^{15} \mathrm{~s}^{-1}$.

- Abschirmkonstanten für Moseley-Gesetz: $\sigma_{K}=1, \sigma_{L}=7,4$ [27].

- Totzeit des Zählrohres $\tau_{D}=100 \mu \mathrm{s}$

Tabelle 22.4: Energieniveaus der Anodenmaterialien.

\begin{tabular}{|c|r|r|r|r|r|}
\hline Anode & $E_{K}$ & $E_{L_{1}}$ & $E_{L_{2}}$ & $E_{L_{3}}$ & $E_{M_{1}}$ \\
\hline Fe (Z=26) & $7112,0 \mathrm{eV}$ & $846,1 \mathrm{eV}$ & $721,1 \mathrm{eV}$ & $708,1 \mathrm{eV}$ & $92,9 \mathrm{eV}$ \\
$\mathrm{Cu}(\mathrm{Z}=29)$ & $8978,9 \mathrm{eV}$ & $1096,1 \mathrm{eV}$ & $951,0 \mathrm{eV}$ & $931,4 \mathrm{eV}$ & $119,8 \mathrm{eV}$ \\
$\mathrm{Mo}(\mathrm{Z}=42)$ & $19999,5 \mathrm{eV}$ & $2865,1 \mathrm{eV}$ & $2625,1 \mathrm{eV}$ & $2520,2 \mathrm{eV}$ & $504,6 \mathrm{eV}$ \\
\hline
\end{tabular}

Tabelle 22.5: Charakteristische Röntgenlinien der Anodenmaterialien.

\begin{tabular}{|c|r|r|r|}
\hline Anode & $E_{K \alpha_{1}}$ & $E_{K \alpha_{2}}$ & $E_{K \beta}$ \\
\hline $\mathrm{Fe}(\mathrm{Z}=26)$ & $6404 \mathrm{eV}$ & $6391 \mathrm{eV}$ & $7058 \mathrm{eV}$ \\
$\mathrm{Cu}(\mathrm{Z}=29)$ & $8048 \mathrm{eV}$ & $8028 \mathrm{eV}$ & $8905 \mathrm{eV}$ \\
$\mathrm{Mo}(\mathrm{Z}=42)$ & $17479 \mathrm{eV}$ & $17374 \mathrm{eV}$ & $19599 \mathrm{eV}$ \\
\hline
\end{tabular}

Tabelle 22.6: K-Absorptionskanten einiger Elemente.

\begin{tabular}{|c|c|c|c|c|c|c|c|c|c|c|c|c|c|}
\hline Element & $\mathrm{Li}$ & $\mathrm{C}$ & $\mathrm{Al}$ & $\mathrm{Si}$ & $\mathrm{Mn}$ & $\mathrm{Fe}$ & $\mathrm{Ni}$ & $\mathrm{Cu}$ & $\mathrm{Zn}$ & $\mathrm{Mo}$ & $\mathrm{Zr}$ & $\mathrm{Sn}$ & $\mathrm{W}$ \\
\hline $\mathrm{Z}$ & 3 & 6 & 13 & 14 & 25 & 26 & 28 & 29 & 30 & 42 & 40 & 50 & 74 \\
\hline$\lambda_{K}[\mathrm{pm}]$ & 22662 & 4365 & 795 & 674 & 190 & 174 & 149 & 138 & 128 & 62 & 69 & 42 & 18 \\
\hline
\end{tabular}

\subsection{Auswertung}

1. Alle Messwerte $N(\theta)$ sind bzgl. der Totzeit des Detektors zu korrigieren (s. Formel (22.2)). Alle weiteren Auswertungen werden mit diesen korrigierten Werten durchgeführt.

2. Das $\mathrm{N}(\theta)$ Spektrum aus Messung 2 ist grafisch aufzutragen. Mit Hilfe der Bragg-Beziehung sind die den Linienmaxima entsprechenden Wellenlängen $\lambda \mathrm{zu}$ berechnen. Man berechne die zugehörigen Energien der charakteristischen Strahlung und ordne den jeweiligen Linien ihre Bezeichnung (z.B. $\mathrm{K}_{\alpha}$ ) und Ordnung n zu.

3. Tragen Sie die gemessenen charakteristischen Linien und die Grenzwellenlängen für unterschiedliche $U_{A}$ in je einem Grafen auf. Bestimmen Sie die Intensität der Linien aus Messung 
3 in Abhängigkeit von der Anodenspannung $\mathrm{U}_{A}$. Bestätigen Sie grafisch das Gesetz 22.8). Berechnen Sie aus den Grenzwellenlängen mit Hilfe des Gesetzes von Duane und Hunt die Plancksche Konstante.

4. Bestimmen Sie aus Messung 4 Lage und Energie der gemessenen Absorptionskanten. Zeichnen Sie dazu die Messwerte halblogarithmisch auf. Berechnen Sie durch das Moseley Gesetz die Rydberg-Konstante unter der Annahme, dass für K-Elektronen die Abschirmkonstante $\sigma \approx 1$ ist.

5. Bestimmen Sie die Absorptionskoeffizienten $\mu / \rho$ der Materialien aus Messung 5 in Abhängigkeit von der Wellenlänge $\lambda$ und tragen Sie die Messwerte $\mathrm{N}(\theta)$ grafisch auf. Verifizieren Sie grafisch die Gültigkeit von $\mu / \rho \sim \lambda^{3}$ im gemessenen Wellenlängenbereich. 


\section{Zeeman-Effekt}

Verschiedene Experimente liefern Hinweise auf eine Eigenschaft des Elektrons, die über dessen Ladung und Masse hinausgeht, welche als Eigendrehimpuls oder Spin bezeichnet wird. Dieser kann, wie auch der Bahndrehimpuls eines Elektrons im Atom, mit einem äußeren Magnetfeld über das zugehörige magnetische Moment wechselwirken, was sich in einer Aufspaltung entarteter Energieniveaus von Atomen äußert. In diesem Versuch wird der normale Zeeman-Effekt an der roten Cadmium-Linie und der anomale Zeeman-Effekt an der grünen Cadmium-Linie im Feld eines Elektromagneten studiert.

\subsection{Stichworte}

Spin, magnetisches Moment des Elektrons, Spektrum in äußeren Feldern, Aufspaltung entarteter Energieniveaus, Fabry-Pérot-Interferometer, Dipol-Auswahlregel.

\subsection{Literatur}

Dem 2 und Dem 3; Gerthsen; BS-3 und BS-4.

\subsection{Zubehör}

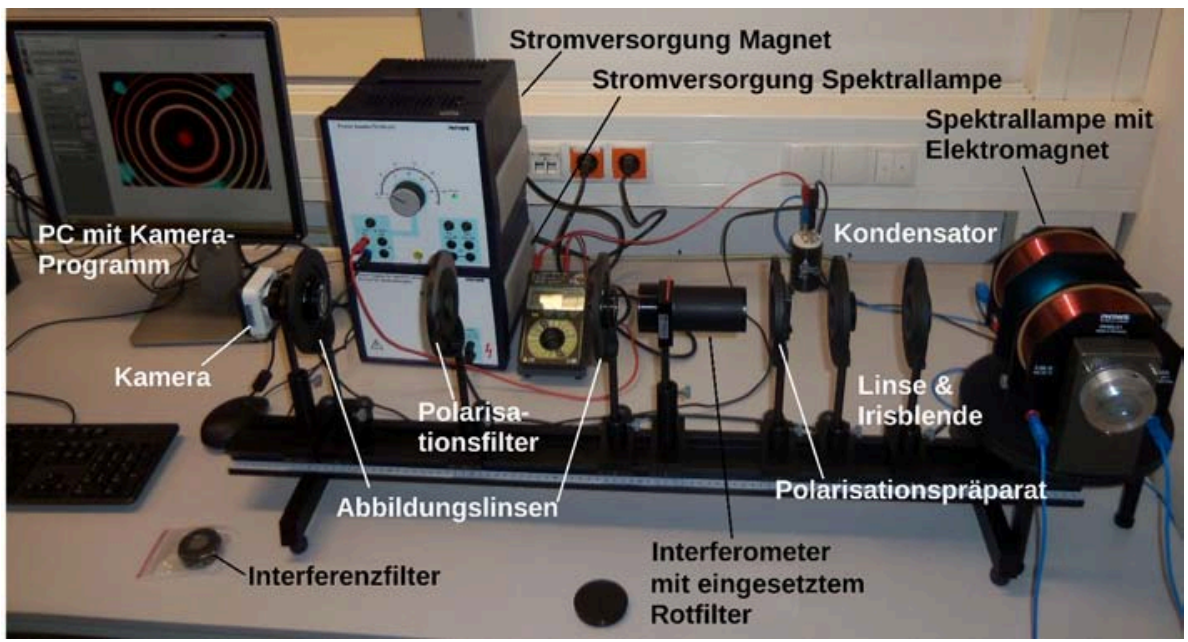

Bild 23.1: Der Versuch »Zeeman-Effekt«. 
Bild 23.1 zeigt ein Foto des Versuchsaufbaus mit Zubehör: Cadmium-Lampe, Elektromagnet auf rotierbarem Tisch, Fabry-Pérot-Interferometer, CCD-Kamera, 3 Linsen, Irisblende, Polarisationsfilter, Polarisationspräparat ( $\lambda / 4$-Plättchen), Rot- bzw. Interferenzfilter zur Wellenlängenselektion.

\subsection{Grundlagen}

\subsubsection{Atome in externen Magnetfeldern}

Analog zu einem klassischen Kreisstrom, der mit magnetischen Effekten einhergeht, muss dem Drehimpuls einer Ladung auch quantenmechanisch ein magnetisches Moment $\vec{p}$ zugeordnet werden. Im Fall eines Elektrons mit Bahndrehimpuls $\vec{l}$ in einem Atom beträgt dies

$$
\vec{p}_{e}=g_{l} \cdot \frac{e}{2 m_{e}} \vec{l}
$$

mit der Elementarladung $e$, der Elektronenmasse $m_{e}$ und dem Landé-Faktor $g_{l}=1$. In einem äußeren magnetischen Feld $\vec{B}$ führt dies zu einer potenzielle Energie

$$
E_{\mathrm{pot}}=-\vec{p}_{e} \cdot \vec{B}
$$

Die auf die Richtung des Magnetfelds projizierte Komponente des Drehimpulses $l_{\| \vec{B}}$ wird durch die magnetische Quantenzahl $m$ beschrieben $(-l \leq m \leq l$, wobei $l$ die Drehimpulsquantenzahl ist):

$$
l_{\| \vec{B}}=m \cdot \hbar,
$$

was mit dem Bohrschen Magneton $\mu_{B}:=\frac{e \hbar}{2 m_{e}}$ für die potenzielle Energie

$$
E_{\mathrm{pot}}=\mu_{B} \cdot m \cdot|\vec{B}|
$$

ergibt. Ist der gesamte Spin des atomaren Zustands Null, so muss nur der Bahndrehimpuls berücksichtigt werden, und G1. (23.4) beschreibt die Verschiebung der Energieniveaus eines Atoms im Magnetfeld $\vec{B}$ : dies wird als normaler Zeeman-Effekt bezeichnet und kann als Aufspaltung der Spektrallinien beobachtet werden.

Bei Berücksichtigung des Elektronenspins $\vec{s}$ ergibt sich eine komplexere Aufspaltung der Energieniveaus und damit der Spektrallinien, welches als anomaler Zeeman-Effekt bezeichnet wird. Hierzu muss der Gesamtdrehimpuls $\vec{j}=\vec{l}+\vec{s}$ betrachtet werden, dessen magnetisches Moment sich zu

$$
\vec{p}_{j}=-g_{j} \cdot \frac{\mu_{B}}{\hbar} \vec{j}
$$

ergibt. Zu beachten ist, dass $\vec{p}_{j}$ i.A. nicht mehr in die Richtung von $\vec{j}$ zeigt. Der zugehörige Landé-Faktor $g_{j}$, der sich über die Drehimpuls- bzw. Spin-Quantenzahlen $l, s$ und $j$ mit dem 
Landé-Faktor des Elektronenspins von $g_{s} \approx 2 \mathrm{zu}$

$$
g_{j}=1+\frac{j(j+1)+s(s+1)-l(l+1)}{2 j(j+1)}
$$

berechnen lässt und die magnetische Quantenzahl $m_{j}$ zu $\vec{j}$ liefern somit für die potenzielle Energie:

$$
E_{\mathrm{pot}}=g_{j} \cdot \mu_{B} \cdot m_{j} \cdot|\vec{B}|
$$

Optische Übergänge zwischen zwei verschiedenen Energie-Zuständen müssen den Dipol-Auswahlregeln folgen. Der vom Photon getragene Drehimpulsunterschied spiegelt sich in der Polarisation wider: Die Übergänge mit $\Delta m_{j}= \pm 1(\pi$-Linien) sind bei Beobachtung transversal zur Richtung des magnetischen Felds vertikal, Übergänge mit $\Delta m_{j}=0$ ( $\sigma$-Linien) sind horizontal polarisiert. Bei Beobachtung parallel zur Richtung des Magnetfelds ist das Licht der $\sigma$-Linien zirkular polarisiert, während die $\pi$-Linien nicht beobachtet werden.

\subsubsection{Fabry-Pérot-Interferometer}

Das Fabry-Pérot-Interferometer ist ein aus zwei teildurchlässigen Spiegeln gebildeter optischer Resonator. Ganz analog zum Resonator, der bei einem Laser eingesetzt wird, kann ein einlaufender Lichtstrahl nur dann durch das Interferometer geleitet werden, wenn die Resonanzbedingung erfüllt ist. So kann nicht nur ein sehr genauer optischer Filter erzeugt werden, sondern wie in diesem Versuch auch ein kleiner Wellenlängenunterschied an Hand des kreisförmigen Interferenzmusters ausgemessen werden.

Durchläuft ein Lichtstrahl der Wellenlänge $\lambda$ unter dem Winkel $\theta_{k}$ das Interferometer der Länge $l$ mit Brechungsindex $n$, so stellt sich konstruktive Interferenz ein, wenn

$$
k \frac{\lambda}{2}=n l \cos \theta_{k}
$$

mit einer ganzen Zahl $k$ gilt. Wird dieser Strahl durch eine Linse der Brennweite $f$ auf eine sich im Abstand von $f$ von der Linse befindenden Ebene abgebildet, so entspricht der Winkel $\theta_{k}$ einem Radius $r_{k}$ gegen die optische Achse von

$$
r_{k}=f \tan \theta_{k} .
$$

Zusammen mit der Interferenzbedingung ergibt sich

$$
r_{k}^{2}=f^{2} \tan ^{2} \theta_{k}=f^{2}\left(\left(\frac{2 n l}{k \lambda}\right)^{2}-1\right) .
$$

Der am nächsten zur optischen Achse liegende Ring mit Radius $r_{1}$ gehört damit zu einem Wert von $k$, welcher der am nächsten zu $\frac{2 n l}{\lambda}$ liegenden ganzen Zahl entspricht. Definieren wir diesen Unterschied zu

$$
\epsilon:=\frac{2 n l}{\lambda}-k\left(r_{1}\right)
$$


so gilt für den von der optischen Achse aus gezählten $p$-ten Ring im Beugungsbild

$$
k\left(r_{p}\right)=\frac{2 n l}{\lambda}-\epsilon-(p-1),
$$

d.h. $k$ wird kleiner mit der Ordnung des Rings. Damit erhalten wir näherungsweise (bei $(\epsilon+p-$ 1) $\left.\frac{\lambda}{2 n l} \ll 1\right)$ für den Radius $r_{p}$ des $p$-ten Rings im Beugungsbild:

$$
r_{p}^{2}=f^{2}\left(\frac{1}{\left(1-(\epsilon+p-1) \frac{\lambda}{2 n l}\right)^{2}}-1\right) \approx f^{2}(\epsilon+p-1) \frac{\lambda}{n l}
$$

Für die quadratische Differenz der Radien zu zwei aufeinander folgenden Ringen ergibt sich somit:

$$
r_{p+1}^{2}-r_{p}^{2} \approx f^{2} \frac{\lambda}{n l}
$$

und kombiniert mit Gl. 23.13):

$$
\frac{r_{p+1}^{2}}{r_{p+1}^{2}-r_{p}^{2}}-p \approx \epsilon
$$

Falls zwei Wellenlängen $\lambda_{1}, \lambda_{2}$ sich nur so geringfügig unterscheiden, dass die Ringe erster Ordnung nicht überlappen, d.h. dass die entsprechenden Ordnungen $k$ gleich sind $\left(k\left(r_{p, 1}\right)=k\left(r_{p, 2}\right)\right)$, so ergibt die Definition von $\epsilon$ in Gl. 23.11:

$$
\epsilon\left(\lambda_{1}\right)-\epsilon\left(\lambda_{2}\right)=\frac{2 n l}{\lambda_{1}}-\frac{2 n l}{\lambda_{2}} .
$$

Zusammen mit Gl. 23.15 ergibt sich folgender Bezug zwischen den gesuchten Wellenlängen und den gemessenen Radien der Beugungsringe:

$$
\frac{1}{\lambda_{1}}-\frac{1}{\lambda_{2}} \approx \frac{1}{2 n l}\left(\frac{r_{(p+1), 1}^{2}}{r_{(p+1), 1}^{2}-r_{p, 1}^{2}}-\frac{r_{(p+1), 2}^{2}}{r_{(p+1), 2}^{2}-r_{p, 2}^{2}}\right),
$$

wobei $r_{i, 1}$ bzw. $r_{i, 2}$ sich auf die Radien zu $\lambda_{1}$ bzw. $\lambda_{2}$ beziehen. Da keine Eigenschaften von Linse und Schirm eingehen und nur Verhältnisse der Radien betrachtet werden, ist weder eine Kalibrierung von $r$ noch eine Vermessung des Teleskops hinter dem Interferometer notwendig.

\subsection{Fragen}

1. Warum gibt es zu Drehimpulsen zwei Quantenzahlen (z.B. $l$ und $m$ ), obwohl es sich um eine vektorielle Größe handelt, d.h. drei Komponenten existieren?

2. Wie wechselwirkt ein externes Magnetfeld mit den Elektronen in einem Atom?

3. Was unterscheidet den normalen vom anomalen Zeeman-Effekt? 
4. Was ist Spin-Bahn-Wechselwirkung?

5. Was besagen die Dipol-Auswahlregeln für optische Übergänge und worin liegt der physikalische Ursprung dieser?

6. Erläutern Sie, wie mit einem Fabry-Pérot-Interferometer ringförmige Beugungsmuster erzeugt werden können.

\subsection{Angaben}

Die bei der Vermessung des normalen Zeeman-Effekts genutzte spektrale Cd-Linie entspricht dem Übergang $3{ }^{1} \mathrm{D}_{2} \rightarrow 2{ }^{1} \mathrm{P}_{1}$ mit einer Wellenlänge von $\lambda=643,85 \mathrm{~nm}$. Beim anomalen ZeemanEffekt entspricht die Linie dem Übergang $2^{3} \mathrm{~S}_{1} \rightarrow 2^{3} \mathrm{P}_{2}$ mit einer Wellenlänge von $\lambda=508,58 \mathrm{~nm}$.

Der Resonanzbereich des Interferometers besteht aus einer $3 \mathrm{~mm}$ dicken Quarzscheibe, dessen Brechungsindex $n=1,4519$ bei $\lambda=509 \mathrm{~nm}$ und $n=1,4560$ bei $\lambda=644 \mathrm{~nm}$ beträgt. Die Quarzscheibe ist an beiden Enden beschichtet, so dass ein 90\%-iger Reflexionsgrad erreicht wird.

Eine graphische Darstellung ist am jeweiligen Versuchsplatz ausgelegt, welche den Verlauf des Magnetfelds in der Mitte der Polschuhe als Funktion des Spulenstroms zeigt.

\subsection{Durchführung}

Das Interferenzmuster des Interferometers wird über ein Teleskop auf eine CCD-Kamera projiziert. Das Teleskop wird aus beiden Linsen linksseitg gebildet (am Interferometer: $f=300 \mathrm{~mm}$, vor der Kamera: $f=50 \mathrm{~mm}$ ). Die CCD-Kamera wird mit dem PC am Messplatz (login: prakt ohne Passwort) über das Programm Motic Images Plus betrieben. Eine Verknüpfung zum Programm und dessen Schnellstartanleitung sind über den Desktop verfügbar. Bei der Einstellung des Aufbaus und bei Änderungen am Magnetfeld sollte das Programm auf permanente Bildaufnahme gestellt werden: Dazu im File-Menü den Eintrag Capture auswählen. Die Einstellungen zu Kontrast, Helligkeit und Sättigung des Bildes sollten so angepasst werden, dass ein deutliches Interferenzmuster zu erkennen ist. Wenn dies erreicht ist, sollte das Bild über das Kamerasymbol in der Fensterleiste oben rechts zwischengespeichert werden; das Bild erscheint anschließend im Hauptfenster des Programms. Dabei ist es sinnvoll, die Parameter der Messung wie die Stromstärke mittels des Text-Werkzeugs einzutragen.

Vor Beginn der Messungen ist die Spektrallampe mit der unteren Stromversorgung zu verbinden. Die Spulen des Elektromagneten sind in Parallelschaltung über ein Strommessgerät mit dem Ausgang der oberen Stromversorgung zu verbinden, der parallel an den $22000 \mu \mathrm{F}$-Kondensator angeschlossen wird. Vor der Aufnahme der Interferenzringe sollte jeweils gewartet werden, bis sich die Stromstärke durch die Spulen stabilisiert hat.

1. Justierung: Der Polarisationsfilter, das Polarisationspräparat und die Irisblende werden von der optischen Bank entfernt. Der Elektromagnet wird so gedreht, dass die Spektrallampe durch eine der Öffnungen in den Polschuhen beobachtet werden kann. Die Linse zwischen Polschuh und Interferometer wird so positioniert, dass sich die Öffnung im Fokus der Linse befindet und der Eingang des Interferometers voll ausgeleuchtet wird. Den Strom der Spule auf 5 A stellen und das Interferenzmuster auf der CCD-Kamera durch Justierung der Linsen zwischen Interferometer und Kamera, dem Fokus an der Kamera und ggf. Verschieben des 
Interferometers scharf und zentriert einstellen. Dieser Aufbau kann in dieser Form für longitudinale Beobachtung verwendet werden. Für transversale Beobachtung den Magneten um $90^{\circ}$ drehen und die Irisblende an den vorherigen Abstand der Polschuhe zur Linse einsetzen, die die Funktion der Polschuhöffnung als Lichtquelle ersetzt.

2. Vermessung des normalen Zeeman-Effekts (transversale Beobachtung):

a. Beide Polarisationsfilter werden nicht verwendet. Der Rotfilter wird in das Interferometer eingesetzt, um die rote Cd-Linie zu selektieren. Das Interferenzfilter wird nicht verwendet. Es werden wie oben beschrieben die Interferenzringe für mindestens fünf verschiedene Werte des Spulenstroms im Bereich $I=4 \ldots 10$ A aufgenommen (über $I=8$ A darf die Spule nur kurzzeitig betrieben werden, s. Bemerkungen) und so viele Ordnungen, wie voneinander unterscheidbare Ringe im Interferenzmuster erkennbar sind, vermessen. Die Ringe sollten sich dabei durch das Magnetfeld in jeweils drei Komponenten aufspalten.

b. Ausmessen der Ringe mit dem Motic-Programm. Mit dem Circle-Eintrag des Menüs Measure können die Interferenzringe vermessen werden: Den Mauszeiger so über das Bild bewegen, dass ein Kreis gezeichnet wird; Position und Größe des Rings sollten dem gewünschten Beugungsring angepasst werden. Nachfolgend werden Radius, Fläche und Umfang des Kreises in einem Kasten im Bild und in der Tabelle unter dem Bild angezeigt; Einheiten und Genauigkeit ggf. anpassen. Da für die Auswertung nur $r^{2}$ benötigt wird, sollte die Fläche verwendet werden. Ergebnis über den Save AsEintrag des File-Menüs z.B. als jpeg-Datei abspeichern.

3. Vermessung des anomalen Zeeman-Effekts (transversale Beobachtung): Der Rotfilter wird entfernt und der Interferenzfilter auf die Linse direkt links vom Interferometer $(f=300 \mathrm{~mm})$ gesteckt. Eine Aufspaltung in sechs Ringe sollte nun beobachtet werden. Messung der Interferenzringe für verschiedene Werte des Spulenstroms analog zum normalen Zeeman-Effekt. Ggf. hilft es, den Polarisationsfilter vor der Linse vor der Kamera einzusetzen und auf $0^{\circ}$ bzw. $90^{\circ}$ einzustellen, um die Ringe besser voneinander unterscheiden zu können.

4. Vermessung der Polarisation des emittierten Lichts in beiden Zeeman-Effekten in transversaler Beobachtung: Der Polarisationsfilter wird zwischen die beiden Linsen hinter dem Interferometer gebracht. Stellen Sie den Spulenstrom auf 5 A. Beobachten Sie einmal mit dem Rotfilter, einmal mit dem Interferenzfilter, welche Ringgruppen bei horizontaler bzw. vertikaler Polarisationsrichtung am Polarisationsfilter $\left(0^{\circ}\right.$ bzw. $\left.90^{\circ}\right)$ beobachtet werden.

5. Vermessung der Polarisation des emittierten Lichts beim anomalen Zeeman-Effekt in longitudinaler Beobachtung: Drehen Sie den Magneten um $90^{\circ}$, so dass die Spektrallampe durch die Polschuhe beobachtet werden kann. Bringen Sie das Polarisationspräparat $(\lambda / 4-$ Plättchen) vor dem Polarisationsfilter in den Strahlengang und entfernen Sie die Irisblende vor dem Interferometer. Beobachten Sie wiederum die Ringgruppen mit Interferenzfilter für $I=5 \mathrm{~A}$, wenn der Polarisationsfilter um $\pm 45^{\circ}$ gedreht wird.

6. Übertragen Sie die Dateien auf einen USB-Stick oder schicken sich diese per Email. Ihr(e) Betreuer(in) erhält hiervon am Versuchstag eine Kopie, was als Teil des Messprotokolls gilt. 


\subsection{Auswertung}

1. Entnehmen Sie die Quantenzahlen des gesamten Bahndrehimpulses, Spins und Gesamtdrehimpulses aus den angegebenen Zuständen des Cd-Atoms bei den beobachteten Übergängen. Verifizieren Sie, dass die Dipol-Auswahlregeln erfüllt sind und dass beim normalen Zeeman-Effekt tatsächlich der Spin verschwindet. Berechnen Sie $g_{j}$ für beide Zustände, die bei der Messung des anomalen Zeeman-Effekts verwendet werden.

2. Bestimmen Sie für beide Zeeman-Effekte die zu erwartende Differenz in $E_{\text {pot }}$ als Funktion von $\mu_{B}$ und $|\vec{B}|$.

3. Verwenden Sie die gemessenen Radien des normalen Zeeman-Effekts, um daraus die Differenz in $\lambda^{-1}$ gemäß Gl. (23.17) zu bestimmen. Mitteln Sie diese über die Ordnungen der Ringe zu dem selben Spulenstrom und tragen Sie das Ergebnis gegen die Stärke des magnetischen Feldes auf und bestimmen Sie aus der Steigung $\mu_{B}$.

4. Wiederholen Sie diese Prozedur für den anomalen Zeeman-Effekt. Bestimmen Sie aus der Steigung von $\Delta \lambda^{-1}$ gegen $|\vec{B}|$ unter der Annahme von $\mu_{B}$ den aus den Landé-Faktoren $g_{j}$ berechneten Proportionalitätsfaktor zur Differenz in $E_{\text {pot }}$.

5. Interpretieren Sie die Beobachtungen der Messungen mit Polarisationsfilter und ordnen Sie die beobachteten Linien den entsprechenden Werten von $\Delta m_{j} \mathrm{zu}$.

\subsection{Bemerkungen}

Die Spulen des Elektromagneten dürfen im Dauerbetrieb mit maximal 4 A versorgt werden. Ein Spulenstrom bis $5 \mathrm{~A}$ darf maximal über einen Zeitraum von 20 Minuten angelegt werden. In Parallelschaltung entspricht dies also einem Strom am Multimeter von max. 8 A im Dauerbetrieb und max. $10 \mathrm{~A}$ bis maximal 20 Minuten. 


\section{Elektronenspinresonanz}

Die Aufhebung der Entartung von Energieniveaus eines Atoms im externen Feld, wie sie im vorhergehenden Kapitel am Zeeman-Effekt erläutert wurde, ist anstelle der Linienaufspaltung auch als direkter Übergang zwischen den Energieniveaus als Elektronenspinresonanz zu beobachten. Dabei werden Atom-Zustände verwendet, in denen der Bahndrehimpuls gegen den Spin des Elektrons, der durch ein äußeres Magnetfeld ausgerichtet wird, vernachlässigbar ist, so dass der Landé-Faktor des Elektronenspins untersucht werden kann.

\subsection{Stichworte}

Spin, magnetisches Moment des Elektrons, Helmholtz-Spule, Schwingkreis, Wechselfeld-Anregung, Dirac-Teilchen, Landé-Faktor.

\subsection{Literatur}

H. Haken und H.C. Wolf: »Atom- und Quantenphysik« [27], Kap. 13.2, 20.5; BS-4.

\subsection{Zubehör}

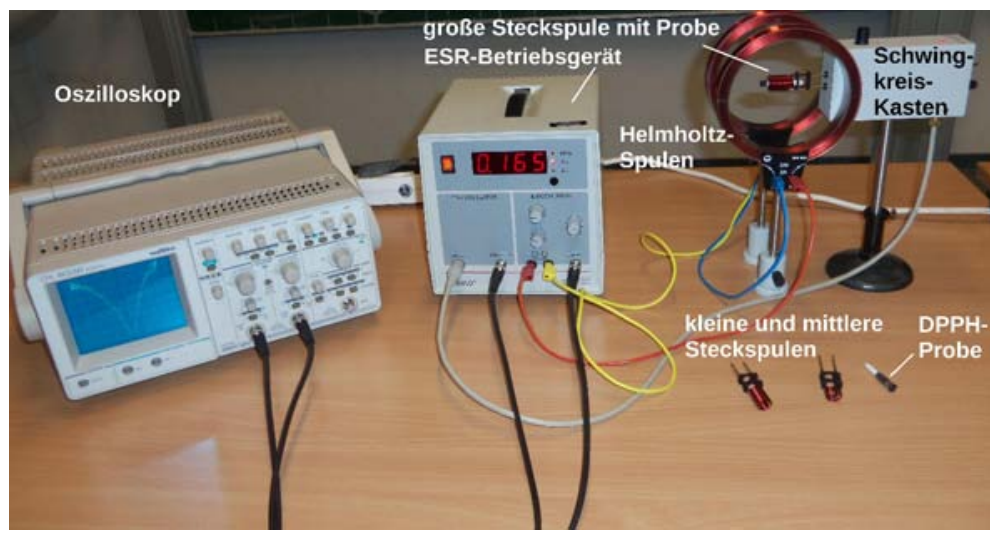

Bild 24.1: Der Versuch »Elektronenspinresonanz«.

Bild 24.1 zeigt ein Foto des Versuchsaufbaus mit Zubehör: ESR-Betriebsgeräte, Steckspulen, DPPH-Probe, Helmholtz-Spulenpaar, Oszilloskop. 


\subsection{Grundlagen}

Analog zur magnetischen Moment, welches beim Zeeman-Effekt zum Bahndrehimpuls bzw. zum Spin-Bahn-Gesamtdrehimpuls betrachtet wurde, ergibt sich für den Spin $\vec{s}$ des Elektrons ein Moment von

$$
\vec{p}_{s}=-g_{s} \cdot \frac{\mu_{B}}{\hbar} \vec{s}
$$

mit dem im vorherigen Abschnitt definierten Bohrschen Magneton $\mu_{B}$ und dem Landé-Faktor $g_{s}$. Daraus ergibt sich wiederum eine potenzielle Energie in einem Magnetfeld $\vec{B}$ über die magnetische Quantenzahl des Spins $m_{s}$ zu

$$
E_{\mathrm{pot}}=g_{s} \cdot \mu_{B} \cdot m_{s} \cdot|\vec{B}| \text {. }
$$

Im Gegensatz zum Bahndrehimpuls, dessen Quantenzahlen ganzzahlig sind, ist die Spinquantenzahl $s=\frac{1}{2}$ und entsprechend die magnetische Quantenzahl ebenfalls halbzahlig: $m_{s}= \pm \frac{1}{2}$. Für den Landé-Faktor des Spins gilt, ebenfalls unterschiedlich zum Bahndrehimpuls, $g_{s} \neq 1$. Im Rahmen der Dirac-Theorie des Elektrons lässt sich dieser zu $g_{s}=2,0023$ berechnen.

Der Übergang zwischen den beiden durch $m_{s}= \pm \frac{1}{2}$ gegebenen Zuständen, d.h. einer Spinausrichtung parallel bzw. antiparallel zum Magnetfeld, erfordert nach Gl. (24.2) eine Energiezufuhr von

$$
\Delta E=g_{s} \cdot \mu_{B} \cdot|\vec{B}|
$$

Überlagert man also dem konstanten Magnetfeld $\vec{B}$ ein Wechselfeld mit der Frequenz $v=\Delta E / h$, so kann der Übergang herbeigeführt werden, was als absorbierte Energie beobachtet werden kann. Dies wird als Elektronenspinresonanz (ESR) bezeichnet.

Die ESR kann an Atomen und Molekülen mit ungepaarten Elektronen beobachtet werden, da dann der Gesamtspin in einer Schale von Null verschieden ist. Dabei handelt es sich also um paramagnetische Stoffe. Die ESR bietet damit nicht nur eine wichtige Möglichkeit zur Untersuchung von Molekülstrukturen, sondern erlaubt auch eine direkte Messung des Landé-Faktor $g_{s}$, was Ziel dieses Versuchs ist. In diesem Versuch wird ein relativ schwaches Magnetfeld im mTBereich verwendet, welches über eine Helmholtz-Spule erzeugt wird. Dies wurde im Versuch »Magnetfeld von Spulen« in Band 1 der Praktikumsanleitung [26] genauer beschrieben und untersucht. Dieses Feld wird zur Verstärkung des zu messenden Effekts mit $50 \mathrm{~Hz}$ moduliert, d.h. mit einer Frequenz, die deutlich unter der Resonanz liegt. Ein solches Magnetfeld entspricht ESRFrequenzen im Bereich $v=10 \ldots 100 \mathrm{MHz}$. Diese werden über einen HF-Schwingkreis erzeugt. Wird die Resonanzbedingung nach Gl. (24.3) erreicht, wird dem Schwingkreis Energie entzogen, und dessen Belastung äußert sich als Änderung der Impedanz des Schwingkreises, was über eine Änderung der an ihm anliegenden Spannung gemessen werden kann.

\subsection{Fragen}

1. Wie unterscheiden sich die möglichen Quantenzahlen eines Bahndrehimpulses und des Spins eines Elektrons? 
2. Welche Werte können die Spinquantenzahl $s$ eines Elektrons und dessen magnetische Quantenzahl $m_{s}$ annehmen? Welche Werte kann der Gesamtspin eines zwei-Elektronen-Systems annehmen?

\subsection{Angaben}

Der Probenkörper besteht aus 1,1-Diphenyl-2-Pikryl-Hydrazyl (DPPH), einer organischen Verbindung mit einem freien Radikal. Das entsprechende Elektron ist näherungsweise vom Molekül unbeeinflusst, so dass der gemessene Landé-Faktor fast dem eines freien Elektrons entspricht $\left(g_{s}^{\mathrm{DPPH}}=2,0036\right)$.

Der Radius der Helmholtz-Spulen beträgt $r=6,8 \mathrm{~cm}$, deren Windungszahl ist $n=320$ (pro Spule).

\subsection{Durchführung}

Verbinden Sie die Helmholtz-Spulen parallel miteinander und mit dem entsprechenden Ausgang des ESR-Betriebsgeräts, siehe Abbildung 24.2 Die Stromstärke kann direkt vom Betriebsgerät

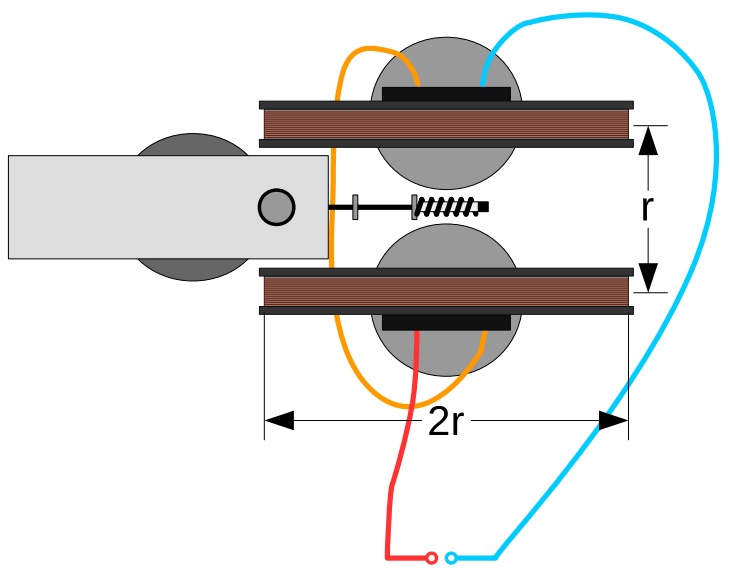

Bild 24.2: Verkabelung der HelmholtzSpulen und Anordnung dieser mit dem Schwingkreis-Kasten und dessen Spule.

abgelesen werden. Die Spulen werden in einem Abstand von 6,8 cm zueinander aufgestellt. Eine der Steckspulen in den Schwingkreis-Kasten stecken (lange Spule: $15 \ldots 30 \mathrm{MHz}$, mittlere Spule: $30 \ldots 75 \mathrm{MHz}$, kurze Spule: $75 \ldots 130 \mathrm{MHz}$ ), DPPH-Probe einbringen und in die Mitte der Helmholtz-Spulen stellen. Schwingkreis-Kasten über das 6-polige Kabel mit dem ESRBetriebsgerät verbinden und letzteres mit den zwei BNC-Koaxialkabeln mit den Eingängen des Oszilloskops verbinden (Ausgang B SIGNAL an Kanal 1, Ausgang ESR SIGNAL an Kanal 2).

1. Resonanzfrequenz auf $15 \mathrm{MHz}$ einstellen (Potentiometer auf dem Schwingkreis-Kasten), die Modulationsamplitude (Potentiometer $I \sim$ am Betriebsgerät) auf mittlere Position stellen, die Phasenverschiebung (Potentiometer Phase) auf $0^{\circ}$ stellen. 
2. Stellen Sie die Resonanzbedingung ein: Mit dem Oszilloskop im ty-Betrieb beide Signale betrachten und mit dem entsprechend Schalter am ESR-Betriebsgerät die Anzeige auf $A=$ stellen, so dass der durch die Spulen fließende Gleichstrom angezeigt wird. Diesen mit dem Potentiometer $I=$ so einstellen, dass die Minima auf der Zeitachse in etwa gleich weit voneinander entfernt sind; dabei den Modulationsstrom so klein wie möglich wählen, um das Erscheinen von »Satelliten «-Minima zu vermeiden. Nun das Oszilloskop auf $x y$-Betrieb stellen und die Phase so einstellen, dass beide Minima in $x$-Richtung überlappen. Nun den Gleichstrom durch die Spulen so nachjustieren, dass das Signal symmetrisch ist.

3. Frequenz in $5 \mathrm{MHz}-\mathrm{Schritten}$ bis $125 \mathrm{MHz}$ einstellen (ggf. Schwingkreisspule wechseln, s.o.) und den Spulenstrom gemäß Punkt 2 nachjustieren und notieren. Die Frequenz kann dabei bei entsprechender Display-Einstellung am ESR-Betriebsgerät abgelesen werden entsprechend zum Gleichstrom durch die Helmholtz-Spulen (s.o.).

\subsection{Auswertung}

1. Bestimmen Sie aus dem bei der Resonanzbedingung gemessenen Spulenstrom das Magnetfeld am Ort der Probe und tragen die Resonanzfrequenz gegen das Magnetfeld auf.

2. Bestimmen Sie aus der Steigung den Landé-Faktor.

\subsection{Bemerkungen}

Die DPPH-Probe ist umschlossen und sollte somit kein Sicherheitsrisiko darstellen. Bei Undichtigkeiten besteht die Gefahr von Sensibilisierung durch Einatmen und Hautkontakt. In diesen Fällen nach dem Einatmen sofort an die frische Luft gehen. Nach Berührung mit der Haut mindestens 15 Minuten mit genügend Wasser abwaschen. Kontaminierte Kleidung und Schuhe wechseln. In beiden Fällen ist ein Arzt zu konsultieren. 


\section{Radioaktivität}

Natürliche und künstliche Radioaktivität spielen eine wichtige Rolle im technischen, gesundheitlichen und politischen Bereich. Für Physikerinnen und Physiker sind Kenntnisse von Strahlungserzeugung und Messung noch wichtiger. Im Rahmen des Praktikums wird der Versuch »Radioaktivität« durchgeführt, in dem die Radioaktivität eines in einer Am-Be Neutronenquelle für verschiedene Zeiträume aktivierten Silber-Plättchens gemessen wird. Die Messung läuft computergesteuert.

\subsection{Stichworte}

Natürliche und künstliche Radioaktivität, radioaktive Zerfälle, Isotope, Zerfallsgesetz, Aktivierungsgesetz, Halbwertszeit, Nachweis ionisierender Strahlung, Geiger-Müller-Zählrohr, Abschirmung radioaktiver Strahlung, $\chi^{2}$-Anpassung

\subsection{Literatur}

BS:4; Gerthsen; Wal; Dem-4; Mayer-Kuckuck: Kernphysik [44]; Musiol: Kern und Elementarteilchenphysik [46]; Povh, Rith, Scholz, Zetsche: Teilchen und Kerne [49]; Frauenfelder, Henley: Teilchen und Kerne [21]; Bevington, Robinson: Data Reduction and Error Analysis for the Physical Sciences [4].

\subsection{Zubehör}

Bild 25.1zeigt ein Foto des Versuches mit Zubehör: Silberplättchen mit den benötigten Isotopen, Am-Be-Quelle zur Aktivierung, Geiger-Müller Zählrohr, Computer mit Datenaufnahmesoftware, Stoppuhr.

\subsection{Grundlagen}

Der Versuch führt in einige Grundlagen der Kernphysik ein und zeigt die typischen Einsatzgebiete von Computern im Bereich der Experimentalphysik. Der Rechner ermöglicht die Messung und Sicherung der Messdaten und nimmt damit dem Experimentator Zeitmessungen mit einer Stoppuhr, das Ein- und Ausschalten der Zählapparatur und die Protokollierung der Daten ab. Daneben soll dieser Versuch auch zeigen, wie die gesicherten Rohdaten mit Rechnerhilfe über eine $\chi^{2}$-Minimierung sehr elegant ausgewertet werden können. Der Praktikumsversuch »Radioaktivität « läuft damit unter Bedingungen $a b$, die in der wissenschaftlichen Forschung gegeben sind. Natürlich müssen die eingesetzten Programme in der Regel für jede spezielle Anwendung vom Experimentator entwickelt und implementiert werden. Zur Datenaufnahme ist für diesen Versuch bereits eine entsprechende Software programmiert worden. Für die Datenanalyse ist Ihnen 


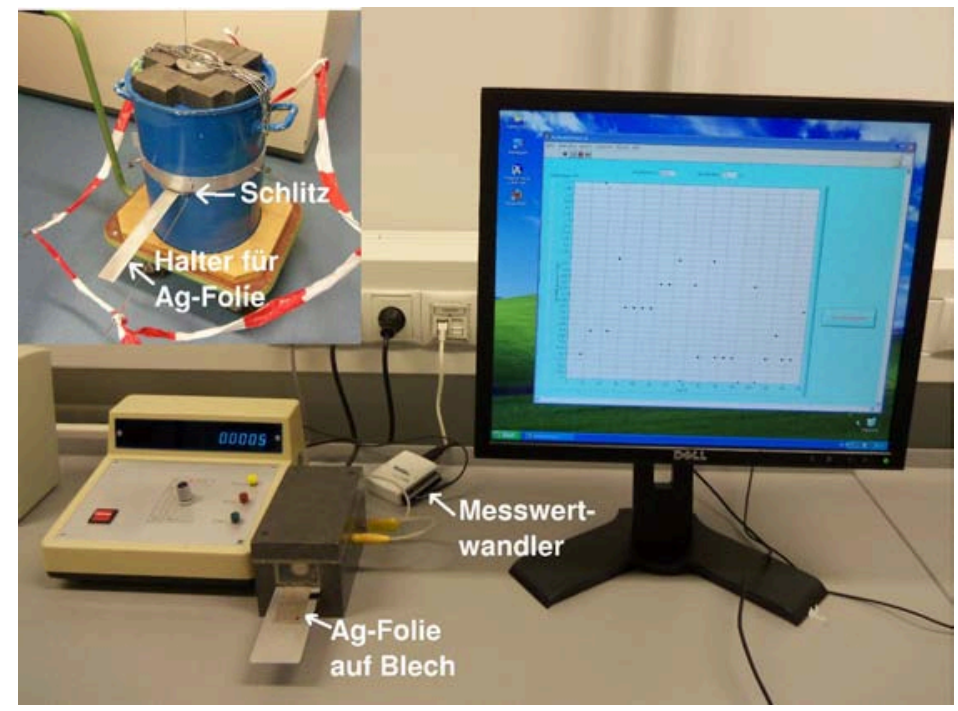

Bild 25.1: Der Versuch »Radioaktivität«. Links oben die Quelle zur Aktivierung.

freigestellt, ob Sie Ihrer Kreativität freien Lauf lassen und eine eigene Analysesoftware entwickeln möchten, oder ob Sie auf Rechnerunterstützung verzichten möchten. Der Versuch soll aber ausdrücklich dazu motivieren, sich mit dem computergestützten $\chi^{2}$-Verfahren zu befassen.

Ziel des Versuches ist es, die Lebensdauern der Silberisotope ${ }_{47}^{108} \mathrm{Ag}$ und ${ }_{47}^{110} \mathrm{Ag}$ über deren häufigsten $\beta$-Zerfall aus den Abklingkurven zu bestimmen. Zur Aktivierung der Proben wird eine Am-Be-Quelle eingesetzt. Die Anfangsaktivität $A_{0}$ der Silberplättchen ist dabei abhängig von der Aktivierungszeit $t_{A}$ : Je länger die Proben in unmittelbarer Nähe der Quelle verbleiben, um so höher ist die Anfangsaktivität. Diese Anfangsaktivität kann nicht beliebig groß werden, sondern hat einen Grenzwert, der mit zunehmender Aktivierungszeit asymptotisch erreicht wird. Die Bestimmung der Grenzwerte beider Isotope aus den Aktivierungskurven bildet den zweiten Teil der Datenanalyse.

\subsubsection{Wechselwirkungen}

Alle beobachteten Kräfte lassen sich vier Wechselwirkungen zuordnen:

- Gravitation

- Elektromagnetische Wechselwirkung

- Schwache Wechselwirkung

- Starke Wechselwirkung

Die Gravitation wirkt zwischen allen massebehafteten Teilchen, kann für diesen Versuch aber wegen den nur sehr kleinen auftretenden Massen vernachlässigt werden. Der Elektromagnetismus wirkt zwischen geladenen Teilchen und wird über ein Photon vermittelt. Damit ist die elektromagnetische Wechselwirkung der prägende Prozess der Physik der Atomhülle, d. h. der Chemie. So geht das Coulombgesetz als elementares Kraftgesetz des Elektromagnetismus bereits in das Bohrsche Modell ein, und führt in der quantenmechanischen Formulierung zwangsläufig zur 
Schalenstruktur der Atome. Die Tatsache, dass Emission oder Absorption von Photonen Übergänge zwischen den Energieniveaus der Atomhülle ermöglichen, ist eine direkte Folgerung der Quantenelektrodynamik (QED), der grundlegenden Theorie der elektromagnetischen Wechselwirkung.

Die fundamentale Bedeutung der schwachen Wechselwirkung besteht darin, dass mit ihr Wechselwirkungen zwischen Objekten beschrieben werden, die nach der klassischen Physik nicht zu erklären sind. Das typische Beispiel ist der $\beta$-Zerfall des Neutrons, an dem Hadronen (Teilchen, die aus Quarks zusammengesetzt sind) und Leptonen (z. B.: e, v) beteiligt sind:

$$
\mathrm{n} \rightarrow \mathrm{p}+\mathrm{e}^{-}+\bar{v}_{\mathrm{e}}
$$

Solch ein Prozess kann im Rahmen des Elektromagnetismus nicht erklärt werden. Der Vollständigkeit halber sei erwähnt, dass auch die schwache Wechselwirkung über eine Quantenfeldtheorie beschrieben werden kann, wobei das $\mathrm{W}^{ \pm}$- und das $\mathrm{Z}^{0}$-Boson die Rolle des Photons übernehmen.

Die starke Wechselwirkung wirkt zwischen einzelnen Quarks unterschiedlicher Farbladung (rot, grün, blau) und wird über die Quantenchromodynamik (QCD) beschrieben. Hier übernehmen farbige Gluonen die Rolle des Photons. Da die Quantenchromodynamik im Bereich der Hadronbindung bis heute numerisch praktisch nicht zu handhaben ist, benutzt man in der Kernphysik eine vereinfachte effektive Wechselwirkung, die Kernkraft. Die Kernkraft wirkt zwischen zwei Nukleonen ( $p$ oder $n$ ) und ist dafür verantwortlich, dass Kerne überhaupt stabil existieren. So sollte ein Heliumkern wegen der elektromagnetischen Abstoßung der beiden Protonen auf den ersten Blick sehr instabil sein. Erst die sehr stark anziehende, zusätzlich wirkende Kernkraft führt zu einem stabilen $\alpha$-Teilchen.

Ebenso wie die elektromagnetische Kraft zwischen Elektronen in der Quantenphysik zur Schalenstruktur der Atomhülle führt, folgt aus der Kernkraft zwischen Nukleonen ein Schalenmodell für Kerne. Die einzelnen Schalen, denen Energieniveaus entsprechen, können mit Protonen und Neutronen aufgefüllt werden (s. Abb. 25.2), wobei besonders stabile, "magische" Kerne (vgl. Edelgaskonfiguration in der Chemie), aber auch instabile Kerne gebildet werden können. Die instabilen Nuklide können dabei über einen radioaktiven Zerfall in andere Kerne umgewandelt werden.

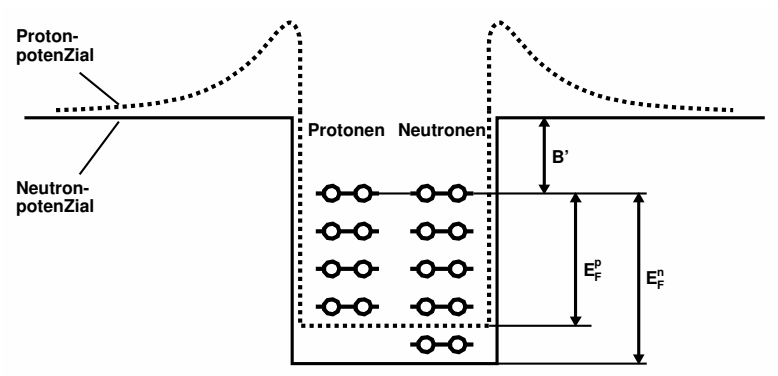

Bild 25.2: Schematischer Verlauf des Potenzials für Proton und Neutron im Fermigasmodell. Das Protonenpotenzial ist leicht erhöht und zeichnet sich durch einen Coulombwall aus, da Protonen als geladene Teilchen auch der elektromagnetischen Kraft unterliegen. 


\subsubsection{Zerfallsarten}

$\alpha$-Zerfall: $\alpha$-Teilchen sind identisch mit dem Nuklid ${ }_{2}^{4} \mathrm{He}$, das eine besonders hohe Stabilität besitzt, da sowohl die Protonenzahl Z=2 und die Neutronenzahl N=2 magischen Zahlen entsprechen. Die allgemeine Reaktionsgleichung lautet

$$
{ }_{\mathrm{Z}}^{\mathrm{A}} \mathrm{X} \rightarrow{ }_{\mathrm{Z}-2}^{\mathrm{A}-4} \mathrm{Y}+{ }_{2}^{4} \mathrm{He}+E_{\alpha, \text { kin }}
$$

wobei X und Y die chemischen Symbole der Nuklide repräsentieren und $E_{\alpha, \text { kin }}$ die kinetische Energie des $\alpha$-Teilchens.

$\boldsymbol{\beta}$-Zerfall: Unter $\beta$-Zerfall versteht man die folgenden Kernreaktionen:

$$
\begin{aligned}
\mathrm{A} X & \rightarrow{ }_{\mathrm{Z}+1}^{\mathrm{A}} \mathrm{Y}+e^{-}+\bar{v}_{e}+E_{\mathrm{kin}}, \\
\mathrm{\textrm {A }} & \rightarrow{ }_{\mathrm{Z}-1}^{\mathrm{A}} \mathrm{Y}+e^{+}+v_{e}+E_{\mathrm{kin}} \quad \text { und } \\
{ }_{\mathrm{Z}}^{\mathrm{A} X}+\mathrm{e}^{-} & \rightarrow{ }_{\mathrm{Z}-1}^{\mathrm{A}} \mathrm{Y}+v_{e}+E_{\mathrm{kin}} .
\end{aligned}
$$

Sie tragen die Bezeichnungen $\beta^{-}$-Zerfall, $\beta^{+}$-Zerfall und Elektroneneinfang und sind elementare Prozesse der schwachen Wechselwirkung. Elektroneneinfang und $\beta^{+}$-Zerfall führen zu dem gleichen Tochternuklid und kommen deshalb häufig nebeneinander vor.

$\gamma$-Zerfall: Die Reaktionsgleichung für den $\gamma$-Zerfall lautet:

$$
{ }_{\mathrm{Z}}^{\mathrm{A}} \mathrm{X}^{*} \rightarrow{ }_{\mathrm{Z}}^{\mathrm{A}} \mathrm{X}+\gamma
$$

Darin weist der Stern $\left(^{*}\right)$ darauf hin, dass sich der Kern in einem angeregten Zustand befindet.

Spontane Spaltung: Sehr schwere Kerne können ohne Energiezufuhr in zwei leichtere Kerne zerfallen, wobei in der Regel 1-3 Neutronen emittiert werden. Diesen Vorgang nennt man spontane Spaltung. Daneben findet die durch Neutroneneinfang ausgelöste neutroneninduzierte Spaltung statt. Wesentlich für diesen Vorgang ist, dass das Neutron durch seinen Einfang eine Bindungsenergie von etwa $8 \mathrm{MeV}$ auf den Kern überträgt und auf diese Weise den Spaltungsvorgang einleitet.

\subsubsection{Die Am-Be-Quelle}

Die Neutronenquelle besteht aus einer Mischung der Nuklide ${ }^{241}$ Am und ${ }^{9} \mathrm{Be}$. In ihr laufen die folgenden Kernreaktionen ab:

$$
{ }_{95}^{241} \mathrm{Am} \stackrel{\mathrm{T}=433}{\longrightarrow}{ }_{93}^{237} \mathrm{~Np}+{ }_{2}^{4} \mathrm{He}+E_{\alpha, \text { kin }},
$$

wobei die kinetische Energie der $\alpha$-Teilchen $E_{\alpha, \text { kin }}=5,5 \mathrm{MeV}$ beträgt. Die energiereichen $\alpha$ Teilchen erzeugen Neutronen über die Reaktionen

$$
{ }_{2}^{4} \mathrm{He}+{ }_{4}^{9} \mathrm{Be} \rightarrow{ }_{6}^{12} \mathrm{C}^{*}+\mathrm{n} \rightarrow{ }_{6}^{12} \mathrm{C}+\gamma+\mathrm{n} .
$$


Die zunächst energiereichen Neutronen werden durch wasserstoffhaltiges Material (hier Paraffin) abgebremst und gesammelt (moderiert) und können dann von den beiden in der Natur gleichzeitig auftretenden stabilen Silberisotopen eingefangen werden:

$$
\begin{aligned}
& { }_{47}^{107} \mathrm{Ag}+\mathrm{n} \rightarrow{ }_{47}^{108} \mathrm{Ag}+\gamma \\
& { }_{47}^{109} \mathrm{Ag}+\mathrm{n} \rightarrow{ }_{47}^{110} \mathrm{Ag}+\gamma
\end{aligned}
$$

Die Nuklide ${ }_{47}^{108} \mathrm{Ag}$ und ${ }_{47}^{110} \mathrm{Ag}$ sind $\beta^{-}$-Strahler mit assoziierter $\gamma$-Strahlung, deren Halbwertzeit gemessen wird. Als Nachweisgerät dient dabei ein Geiger-Müller-Zählrohr, wie es auch für die Röntgenröhre eingesetzt wird (Beschreibung siehe Versuch Röntgenstrahlung).

\subsubsection{Das Zerfallsgesetz}

Für den radioaktiven Zerfall gilt das Zerfallsgesetz

$$
\dot{N}(t)=\dot{N}_{0} \cdot e^{-\lambda t} .
$$

Darin bedeutet $\dot{N}(t)$ die Zerfallsrate (Anzahl pro Zeiteinheit) zum Zeitpunkt $t, \dot{N}_{0}=\dot{N}(t=0)$ die Zerfallsrate unmittelbar nach Abschluss der Aktivierung und $\lambda$ die Zerfallskonstante. Für die Anfangszerfallsrate $\dot{N}_{0}$ gilt das Aktivierungsgesetz

$$
\dot{N}_{0}(\tau)=\dot{N}_{0}^{\infty}\left(1-e^{-\lambda \tau}\right)
$$

worin $\tau$ die Aktivierungszeit bedeutet. Die Halbwertszeit $T_{1 / 2}$ ist definiert als diejenige Zeit, nach der im Mittel gerade die Hälfte aller aktiven Kerne zerfallen sind. Damit gilt:

$$
\lambda=\frac{\ln 2}{T_{1 / 2}} .
$$

\subsection{Fragen}

1. Wie hängt die mittlere Bindungsenergie pro Nukleon von der Massenzahl ab?

2. Wie kann man die Gleichungen (25.11, 25.12) und 25.13) herleiten?

3. Wie lassen sich die unterschiedlichen Arten von Strahlen $(\alpha, \beta, \gamma, n)$ abschirmen?

4. Was ist der Nulleffekt (auch Nullrate), woraus setzt er sich zusammen?

\subsection{Durchführung}

Achtung: Man beachte die Hinweise des Assistenten zum Umgang mit radioaktiven Präparaten und mache sich vor der ersten Messung mit der Durchführung des Versuches vertraut! Es ist verboten während dieses Versuches zu essen, zu trinken oder zu rauchen. Es empfiehlt sich nach Abschluss des Versuches die Hände zu waschen. 
Im Versuch werden die Abklingkurven des zuvor in der Quelle für 1 Minute (bzw. 2, 4, 8 Minuten) aufaktivierten Silberplättchens gemessen. Abschließend wird die Nullrate der natürlichen Strahlung gemessen. Insgesamt sind also fünf Messreihen aufzunehmen.

\subsubsection{Vorbereitung}

Bevor eine Messung durchgeführt werden kann, muss der PC korrekt mit dem Messgerät verbunden sein, was normalerweise der Fall sein sollte. Sonst reicht es, die LEMO-Eingangsleitung der NI-USB-6009 Messwertwandlers am PC mit der entsprechenden Buchse des Messgerätes zu verbinden.

Zur Messung muss das Messgerät eingeschaltet sein, der Timer sollte auf »Aus« stehen und die Messung mit »Start « gestartet werden. Das Gerät kann nun für den Rest des Versuchs ignoriert werden, von der Kontrolle der Aktivität über den Zähler einmal abgesehen.

Schalten Sie den Praktikumsrechner ein (Windows) und melden Sie sich am Rechner an (Login: prakt, kein Passwort). Starten Sie das Programm »Radioaktivität« (Icon auf dem Desktop). Die Bedienung dieses Messprogramms sollte eigentlich recht intuitiv sein.

\subsubsection{Aufaktivierung des Silberplättchens}

Ein nicht mehr aktives Silberplättchen wird mit der Pinzette auf den langen Metallträger gesetzt und vorsichtig durch die schmale Öffnung in der Vorderseite der Abschirmung der Quelle eingeführt 11 Die Probe wird so in die Nähe der Am-Be-Probe gebracht und die Neutronenbestrahlung des Plättchens - und damit auch die Aktivierung - beginnt. Starten Sie sofort nach dem Einschieben die Stoppuhr, die die Aktivierungszeit misst.

\subsubsection{Datennahme}

Die Datenaufnahme erfolgt mit einem speziell für diesen Versuch entwickelten LABVIEW-Programm.

Beim Zerfall der radioaktiven Probe wird die entstehende Gammastrahlung (und gleichzeitig auch die immer vorhandene natürliche Strahlung) vom Geiger-Müller-Zählrohr registriert und in einen Spannungsimpuls umgesetzt. Dieser Impuls wird auf eine digitale Eingabekarte am PC gegeben, die von der Datenaufnahmesoftware Radioaktivität ausgelesen wird. Das Programm zählt dabei die in einem vorgegebenen 5 s-Zeitintervall (Bin) vom Zählrohr registrierten Ereignisse und trägt den ermittelten Wert graphisch auf der Benutzeroberfläche auf.

Führen Sie die folgenden Schritte aus:

1. Nach genau 1 Minute (bzw. 2, 4, 8 Minuten) wird die Probe vorsichtig aus der Quelle entfernt. In dem Moment, in dem Sie nun eine aktivierte Probe aus der Quelle ziehen, starten Sie am Computer eine neue Messung über die Schaltfläche »Zeit $\operatorname{starten} \ll 2$

1 Die Neutronenquelle ist in einem abgeschirmten, blauen Fass eingebaut, welches zu Praktikumsbeginn in den gegenüberliegenden Raum gestellt wird. Dort sollte auch der Metallträger mit Pinzette zu finden sein. Der Metallträger mit Ag-Plattchen wird dann in den seitlichen Schlitz eingeschoben.

2 Vorher vorbereiten. 
2. Dann wird das aktivierte Plättchen mit der Pinzette vom langen auf den kurzen Metallhalter umgesetzt. Mit diesem kurzen Halter führt man das aufaktivierte Silberplättchen nun in das mit Blei abgeschirmte Geiger-Müller-Zählrohr neben dem Computer ein. Zusammen mit der Einführung der Probe in das Zählrohr wird nun die Messung selbst durch einen Klick auf »Messung starten $\ll$ gestartet

3. Wenn Sie genügend Messwerte haben, der Aktivitätsverlauf also bis zum Schluss gut zu erkennen ist, beenden Sie die Messung mit »Messung beenden«.

4. Nun können Sie die Messwerte über die Schaltfläche »Drucken« auf dem angeschlossenen Drucker ausgeben und über »Daten speichern « auf der Festplatte speichern. Bitte legen Sie bei der ersten Messung ein Verzeichnis mit den aktuellen Datum an und lassen alles andere unverändert. Die Ausgabe in der Datei besteht nun aus vielen Messpunkten (Zeilen), in denen die erste Spalte die bis dahin vergangene Zeit angibt und die zweite Spalte die gemessene Anzahl der Zerfallsteilchen der letzten 5 Sekunden 3 .

5. Beachten Sie: Der Nullpunkt für die Zeit ist der Moment, in dem die Probe aus der Quelle gezogen wird!

6. Wiederholen Sie diese Schritte für alle Messreihen (alle Aktivierungszeiten).

\subsection{Angaben}

Die Halbwertszeiten der Isotope lauten: ${ }_{47}^{110} \mathrm{Ag}: T_{\mathrm{A}}=24,6 \mathrm{~s} ; \quad{ }_{47}^{108} \mathrm{Ag}: T_{\mathrm{B}}=143 \mathrm{~s} ; \quad{ }_{95}^{241} \mathrm{Am}:$ $T=432,2$ y.

\subsection{Auswertung}

Im Versuch sind fünf Datenreihen gemessen worden, z. B. 1min.dat, 2min.dat, 4min.dat, 8min. dat und nullrate. dat. Aufgabe der Analyse ist es nun, aus diesen Rohdaten die Zerfallskurven, Aktivierungskurven, Halbwertszeiten und maximalen Anfangsaktivitäten der beiden radioaktiven Silberisotope zu bestimmen. Hauptschwierigkeit dabei ist, dass die aufgenommenen Zählraten eine Überlagerung zweier Zerfälle sind, die nicht unmittelbar zu trennen sind.

Im Folgenden werden zwei alternative Analysemethoden vorgestellt: Die erste ist ein eher ungenaues schrittweises Verfahren, das sich ohne Rechnerunterstützung durchführen lässt. Die zweite Methode basiert auf einer $\chi^{2}$-Minimierung, die nur mit Computerhilfe ausführbar ist. Den PraktikantInnen bleibt überlassen, nach welchem der beiden Verfahren (oder vielleicht einer dritten Methode?) die Daten analysiert werden. An dieser Stelle soll lediglich dazu ermuntert werden, sich in die $\chi^{2}$-Methode einzuarbeiten, da dieses Verfahren universell, d. h. auch für andere Versuchsauswertungen, einsetzbar ist und sich in der Praxis etabliert und bewährt hat.

Da man es in diesen Versuch mit der Summe zweier Exponentialfunktionen zu tun hat, wird empfohlen, sich vor der Auswertung die Logarithmengesetze in Erinnerung zu rufen.

3 NB: Graphisch wird die Aktivität, d.h. Zähler pro Zeit, aufgetragen, während die Datei nur den Zählerstand enthält 


\subsubsection{Die »klassische« Methode}

1. Bestimmung der statistischen Messunsicherheit jedes einzelnen Messwertes:

Da der radioaktive Zerfall der Poisson-Statistik gehorcht, ist der Messfehler der Ereigniszahl $N$ als $\sqrt{N}$ gegeben.

2. Bestimmung der Nullrate aus der Nullratenmessung.

3. Korrektur der Zählraten um die Nullrate. Wie ändert sich die Unsicherheit der einzelnen Zählraten?

4. Grafische Darstellung (linear und halblogarithmisch) der um die Nullrate korrigierten Abklingkurven in Abhängigkeit von der Zeit. Man zeichne die zur jeder Einzelmessung gehörige statistische Unsicherheit als Fehlerbalken ein.

5. In der logarithmischen Auftragung zeigen sich die beiden Isotope jeweils als Geraden. Unter der Annahme, dass das kurzlebige Isotop (A) sehr schnell abklingt, lassen sich für hohe Zeiten Geraden an die Abklingkurven anpassen. Die y-Achsenabschnitte dieser Geraden geben dann jeweils den Logarithmus der Anfangsaktivität für das langlebige Isotop (B) an. Aus den Steigungen extrahiere man die Halbwertszeiten $T_{\mathrm{B}}^{1 \min }, T_{\mathrm{B}}^{2 \min }, T_{\mathrm{B}}^{4 \min }$ und $T_{\mathrm{B}}^{8 \min }$, die anschließend gewichtet zu mitteln sind.

In diesem Auswertungsteil sollen also insgesamt fünf Größen (plus Fehler) bestimmt werden: Die Anfangsaktivitäten $\dot{N}_{B, 0}^{1 \text { min }}, \dot{N}_{\mathrm{B}, 0}^{2 \min }, \dot{N}_{B, 0}^{4 \min }, \dot{N}_{B, 0}^{8 \text { min }}$ und die gewichtete Halbwertszeit $T_{\mathrm{B}}$.

6. Man subtrahiere aus den um die Nullrate korrigierten Abklingkurven (in linearer Darstellung) jeweils die berechneten Abklingkurven für das langlebige Isotop (B) und stelle das Ergebnis in logarithmischer Darstellung grafisch dar.

7. Aus den um die Nullrate und Isotop (B) korrigierten logarithmischen Abklingkurven bestimme man durch Anpassen einer Geraden die Anfangsaktivitäten (plus Fehler) $\dot{N}_{A, 0}^{1 \min }, \dot{N}_{\mathrm{A}, 0}^{2 \min }$, $\dot{N}_{A, 0}^{4} \min , \dot{N}_{\mathrm{A}, 0}^{8 \min }$ und die Halbwertzeit (plus Fehler) $T_{\mathrm{A}}$ für das kurzlebige Isotop (A).

8. Man zeichne in der Grafiken aus Teil 5 die nun einzeln bekannten Abklingkurven für Isotop (A) und (B) mit ein.

9. Bestimmung der Aktivierungskurve für Isotop (A):

Die vier Anfangsaktivitäten $\dot{N}_{A, 0}^{1 \text { min }}, \dot{N}_{A, 0}^{2 \min }, \dot{N}_{A, 0}^{4}$ min und $\dot{N}_{A, 0}^{8 \text { min }}$ sind in Abhängigkeit von der Aktivierungszeit inklusive Fehlerbalken grafisch darzustellen. Man bestimme den asymptotischen Grenzwert für unendlich lange Aktivierungszeit $\dot{N}_{A, 0}^{\infty}$.

10. Grafische Darstellung der Aktivierungskurve und Bestimmung der maximalen Anfangsaktivität $\dot{N}_{B, 0}^{\infty}$ für Isotop (B).

11. Vergleich der experimentell ermittelten Halbswertzeiten mit den Literaturwerten. Diskussion der Ergebnisse. 


\subsubsection{Die $\chi^{2}$ Methode}

1. Umrechnung der Rohdaten in physikalische, fehlerbehaftete Größen analog zu Teil 1 und 2 der »klassischen Methode $\ll$.

2. Grafische Darstellung (linear und logarithmisch) der Abklingkurven und der Nullratenmessung in Abhängigkeit von der Zeit inklusive Fehlerbalken. Die Abklingkurven brauchen im Gegensatz zu Teil 3 der »klassischen Methode« hier nicht um die Nullrate korrigiert zu werden.

3. Bestimmung der Nullrate $\dot{N}^{\text {Null }}$, der Anfangsaktivitäten $\dot{N}_{A, 0}^{1 \min }, \dot{N}_{\mathrm{A}, 0}^{2 \min }, \dot{N}_{A, 0}^{4 \min }, \dot{N}_{\mathrm{A}, 0}^{8 \min }, \dot{N}_{B, 0}^{1 \min }$, $\dot{N}_{\mathrm{B}, 0}^{2 \min }, \dot{N}_{B, 0}^{4} \min , \dot{N}_{B, 0}^{8 \min }$ und der Halbwertszeiten $T_{\mathrm{A}}, T_{\mathrm{B}}$ der beiden Isotope (A) und (B) über eine simultane $\chi^{2}$-Anpassung an alle Messdaten:

Dieser Versuch lässt sich über eine $\chi^{2}$-Minimierung auswerten, da der funktionale Zusammenhang $f(t)$ der Abklingkurven bekannt ist. Konkret handelt es sich um eine Summe aus einer Exponentialfunktion für Isotop (A), einer Exponentialfunktion für Isotop (B) und der konstanten Nullrate mit

$f(t)= \begin{cases}\dot{N}_{A, 0}^{1 \min } \cdot e^{-\frac{\ln 2}{T_{\mathrm{A}}} t}+\dot{N}_{B, 0}^{1 \min } \cdot e^{-\frac{\ln 2}{T_{\mathrm{B}}} t}+\dot{N}^{\text {Null }} & \text { für } 1 \text { Minute Aktivierungszeit, } \\ \dot{N}_{A, 0}^{2 \min } \cdot e^{-\frac{\ln 2}{T_{\mathrm{A}}} t}+\dot{N}_{B, 0}^{2 \min } \cdot e^{-\frac{\ln 2}{T_{\mathrm{B}}} t}+\dot{N}^{\text {Null }} & \text { für } 2 \text { Minuten Aktivierungszeit, } \\ \dot{N}_{A, 0}^{4 \min } \cdot e^{-\frac{\ln 2}{T_{\mathrm{A}}} t}+\dot{N}_{B, 0}^{4 \min } \cdot e^{-\frac{\ln 2}{T_{\mathrm{B}}} t}+\dot{N}^{\text {Null }} & \text { für } 4 \text { Minuten Aktivierungszeit, } \\ \dot{N}_{A, 0}^{8 \min } \cdot e^{-\frac{\ln 2}{T_{\mathrm{A}}} t}+\dot{N}_{B, 0}^{8 \min } \cdot e^{-\frac{\ln 2}{T_{\mathrm{B}}} t}+\dot{N}^{\text {Null }} & \text { für } 8 \text { Minuten Aktivierungszeit und } \\ \dot{N}^{\text {Null }} & \text { für die Nullratenmessung. }\end{cases}$

Diese Formulierung beinhaltet die Bedingungen, dass die Halbwertszeiten der beiden Isotope jeweils für alle Messungen gleich sein müssen, und dass die Nullrate für alle Messungen konstant ist. Aufgabe des $\chi^{2}$-Verfahrens ist es nun, die 11 Parameter und deren statistische Unsicherheiten so zu bestimmen, dass $f(t)$ die gemessenen Daten möglichst optimal (im Sinne der Statistik) beschreibt.

Dazu wird eine Funktion $\chi^{2}$ definiert, die die Übereinstimmung zwischen Theorie und Experiment in Form der quadratischen Abweichung zwischen $f(t)$ und den gemessenen Daten $y$ unter Berücksichtigung des Messfehlers $\sigma_{y}$ und des Modellfehlers $\sigma_{f\left(t_{i}\right)}$ quantifiziert:

$$
\chi^{2}:=\sum_{i} \frac{\left(f\left(t_{i}\right)-y_{i}\right)^{2}}{\sigma_{f\left(t_{i}\right)}^{2}+\sigma_{y_{i}}^{2}}
$$

Da der radioaktive Zerfall der Poisson-Statistik gehorcht, gilt für diesen Versuch $\sigma_{y_{i}}=\sqrt{y_{i}}$. Der Modellfehler der Theorie wird für diesen Versuchsteil als $\sigma_{f\left(t_{i}\right)}=0$ angenommen. Die Summe läuft über alle Messpunkte $i=1, \ldots, n$.

$\chi^{2}$ ist für diesen Versuchsteil also eine 11-dimensionale Funktion der gesuchten Parameter. Es ist anhand der Definition klar, dass derjenige Parametersatz das Experiment optimal beschreibt, bei dem $\chi^{2}$ gerade minimal wird. Die Hauptaufgabe besteht also darin, dieses Minimum numerisch zu bestimmen, wozu man in der Regel auf bekannte Algorithmen zurückgreift (z. B. Marquardt-Levenberg (gnuplot, xmgrace), siehe Webseiten). 
Um die statistische Unsicherheit eines so bestimmten Parameters zu ermitteln, wird der entsprechende Parameter ausgehend vom $\chi^{2}$-Minimum so lange variiert, bis sich $\chi^{2}$ gerade um 1 vergrößert. Die dafür nötige Variation des Parameters entspricht dann gerade seinem Fehler.

Die Zulässigkeit der $\chi^{2}$-Methode setzt voraus, dass $f(t)$ den funktionalen Zusammenhang der Messdaten richtig beschreibt und in unserem Fall die Daten tatsächlich nach der PoissonStatistik verteilt sind. Um diese Annahmen zu testen, betrachtet man abschließend das reduzierte $\chi^{2}$,

$$
\chi_{\text {red }}^{2}:=\frac{\text { minimales } \chi^{2}}{\text { Anzahl der Freiheitsgrade }} .
$$

Die Anzahl der Freiheitsgrade ist dabei gerade die Anzahl der Datenpunkte minus die Anzahl der Parameter. Nach den Gesetzen der statistischen Mathematik sollte sich für $\chi_{\text {red }}^{2}$ ein Wert von 1,0 ergeben. Ist dies nicht der Fall, so deutet dies auf eine Verletzung einer oder mehrerer Annahmen hin.

In der Auswertung bestimme man die 11 Parameter inklusive Fehler nach dem beschriebenen oder einem analogen Verfahren und diskutiere die Ergebnisse anhand des reduzierten $\chi^{2}$. Für die Berechnungen kann bei Bedarf auf vorhandene Software zurückgegriffen werden.

4. Man zeichne in die Grafiken aus Teil 2 die jeweiligen Abklingkurven für Isotop (A) und (B), die Nullrate, sowie die Summe $f(t)$ ein.

5. Bestimmung der Aktivierungskurve für Isotop (A): Die vier Anfangsaktivitäten $\dot{N}_{A, 0}^{1} \min , \dot{N}_{A, 0}^{2 \min }$, $\dot{N}_{A, 0}^{4}$ min und $\dot{N}_{A, 0}^{8 \text { min }}$ sind in Abhängigkeit von der Aktivierungszeit $\tau$ inklusive Fehlerbalken grafisch darzustellen.

Der asymptotische Grenzwert für unendlich lange Aktivierungszeit $\dot{N}_{\mathrm{A}, 0}^{\infty}$ wird über eine $\chi^{2}$ Anpassung mit $f(\tau)=\dot{N}_{\mathrm{A}, 0}^{\infty}\left(1-\exp \left(-\frac{\ln 2}{T_{\mathrm{A}}} \tau\right)\right)$ ermittelt. Zu beachten ist, dass $\sigma_{f\left(\tau_{i}\right)} \neq 0$ ist, da die Halbwertzeit fehlerbehaftet ist. Im Gegensatz zu Teil 3 ist hier nur das Minimum einer eindimensionalen Funktion zu bestimmen, was numerisch erheblich einfacher ist.

Der ermittelte Wert für $\dot{N}_{\mathrm{A}, 0}^{\infty}$ ist in die Grafik mit einzuzeichnen und das reduzierte $\chi^{2}$ ist zu diskutieren.

6. Auswertung der Aktivierungskurve für Isotop (B) analog zu Teil 5.

7. Vergleich der Halbwertszeiten mit den Literaturwerten. Diskussion der Ergebnisse.

\subsection{Bemerkungen}

Die Silberplättchen dürfen nicht mit den Fingern angefasst werden, bitte ausschließlich die beiliegenden Pinzetten benutzen. An der Quelle dürfen außer der Aktivierung keinerlei weitere Arbeiten durchgeführt werden (auch nicht von der Betreuerin oder dem Betreuer)! 
Anhang 


\section{Literaturverzeichnis}

[1] Alonso, Marcelo und E. J. Finn: Physik. Oldenbourg, München, 2000.

[2] Becker, J. und H. J. Jodl: Physik Für Ingenieure und Naturwissenschaftler. VDI Verlag, Düsseldorf, 1991. ISBN: 3-18-400939-4.

[3] Berber, J., H. Kacher und R. Langer: Physik in Formeln und Tabellen. Teubner, Stuttgart, 1986.

[4] Bevington, Philip R. und D. Keith Robinson: Data Reduction and Error Analysis for the Physical Sciences. McGraw-Hill, 2. Auflage, 1992. 0-07-911243-9.

[5] Bronstein, I. N., K. A. Semendjajew, G. Musiol und H. Mühlig: Taschenbuch der Mathematik. Harri Deutsch, Thun, Frankfurt, 4. Auflage, 1999.

[6] Cohen, E. Richard und Pierre Giacomo: Symbols, Units, Nomenclature and Fundamental Constants in Physics. IUPAP-25, CODATA. IUPAP - International Union of Pure and Applied Physics, Thousand Oaks, 1987. Reprinted from Physica 146A (1987) 1-68.

[7] Cornay, A.: Atomic and Laser Spectroscopy. Clarendon Press, Oxford, 1999.

[8] Crawford, Frank S.: Schwingungen und Wellen, Band 3 der Reihe Berkeley-Physik-Kurs. Vieweg, Braunschweig, 1989.

[9] Demtröder, Wolfgang: Experimentalphysik 3: Atome, Moleküle und Festkörper. Springer-Lehrbuch. Springer, Berlin Heidelberg New York, 2010. ISBN: 978-3-642-03910-2.

[10] Demtröder, Wolfgang: Experimentalphysik 4: Kern-, Teilchen- und Astrophysik. Springer-Lehrbuch. Springer, Berlin Heidelberg New York, 2010. ISBN: 978-3-642-01597-7.

[11] Demtröder, Wolfgang: Experimentalphysik 1: Mechanik und Wärme. Springer-Lehrbuch. Springer, Berlin Heidelberg, 2013. ISBN: 978-3-642-25465-9.

[12] Demtröder, Wolfgang: Experimentalphysik 2: Elektrizität und Optik. Springer-Lehrbuch. Springer, Berlin Heidelberg, 2013. ISBN: 978-3-642-29943-8.

[13] Diemer, U., B. Basel und H. J. Jodl: Computer im Praktikum. Springer, Berlin, 1999.

[14] Dories, Ralf und Heinz Schmidt-Walter: Taschenbuch der Elektrotechnik. Harri Deutsch, Thun, Frankfurt, 1998.

[15] Dorn, Friedrich und Franz Bader: Physik in Einem Band, Sek II. Schroedel Verlag, Hannover, 2000.

[16] Drosg, Manfred: Der Umgang mit Unsicherheiten. facultas, Wien, 2006.

[17] Eichler, H. J., H. D. Kronfeldt und J. Sahm: Das Neue Physikalische Grundpraktikum. Springer Lehrbuch. Springer, Berlin Heidelberg New York, 2006. 978-3-540-21453-3. 
[18] Feynman, Richard P., Robert B. Leighton und Matthew Sands: Elektromagnetismus und Struktur der Materie, Band 2 der Reihe Feynman Lectures in Physics. Oldenbourg, 1999-2001.

[19] Feynman, Richard P., Robert B. Leighton und Matthew Sands: Mechanik, Strahlung, Wärme, Band 1 der Reihe Feynman Lectures in Physics. Oldenbourg, 1999-2001.

[20] Feynman, Richard P., Robert B. Leighton und Matthew Sands: Quantenmechanik, Band 3 der Reihe Feynman Lectures in Physics. Oldenbourg, 1999-2001.

[21] Frauenfelder, Hans und Ernest M. Henley: Teilchen und Kerne: Die Welt der Subatomaren Physik. Oldenbourg, München, 1999.

[22] Grimsehl, Ernst: Elektromagnetisches Feld, Band 2 der Reihe Lehrbuch der Physik. Teubner, Leipzig, 1967.

[23] Grimsehl, Ernst: Struktur der Materie, Band 4 der Reihe Lehrbuch der Physik. Teubner, Leipzig, 1968.

[24] Grimsehl, Ernst: Optik, Band 3 der Reihe Lehrbuch der Physik. Teubner, Leipzig, 1969.

[25] Grimsehl, Ernst: Mechanik, Akustik, Wärmelehre, Band 1 der Reihe Lehrbuch der Physik. Teubner, Leipzig, 1971.

[26] Große-Knetter, J. und P. Schaaf: Das Physikalische Praktikum, Band 1. Universitätsverlag Göttingen, 2015.

[27] Haken, H. und H. C. Wolf: Atom- und Quantenphysik. Springer, Berlin, 2004. ISBN: 3-540-02621-5.

[28] Halliday, David, Robert Resnick und Jearl Walker: Physik. Wiley VCH, Weinheim, 2003. Deutsche Bearbeitung: Stephan W. Koch; ISBN: 3-527-40366-3.

[29] Hermbecker, K.: Handbook of Physics X-Ray Experiments. Phywe, Göttingen, 2002.

[30] Kittel, Charles, Walter D. Knight, Malvin A. Ruderman und Robin Pestel: Mechanik, Band 1 der Reihe Berkeley-Physik-Kurs. Vieweg, Braunschweig, 1991.

[31] Kneubühl, F. K.: Repetitorium der Physik. Teubner, Stuttgart, 1988.

[32] Kneubühl, F. K. und M. W. Sigrist: Laser. Teubner Studienbücher. Teubner, Stuttgart, 4. Auflage, 1995.

[33] Kohlrausch, Friedrich: Praktische Physik 1: Mechanik, Akustik, Wärme, Elektrizität. Teubner, Stuttgart, 24. Auflage, 1996.

[34] Kohlrausch, Friedrich: Praktische Physik 2: Magnetismus, Optik, Radioaktivität, Strahlung, Struktur und Eigenschaften Von Materie. Teubner, Stuttgart, 24. Auflage, 1996.

[35] Kohlrausch, Friedrich: Praktische Physik 3: Messung und Auswertung, Elektronik, Messdatenerfassung, Normung, Prüfwesen. Teubner, Stuttgart, 24. Auflage, 1996.

[36] Kuhn, H. G.: Atomic Spectra. Longmans, London, 1962.

[37] Kunze, H. J.: Physikalische Meßmethoden. Teubner Studienbücher Physik. Teubner, Stuttgart, 1986. 
[38] Lichten, W. und D. Meschede: Skriptum Fehlerrechnung. Springer, Heidelberg, 1988.

[39] Lipson, S. G., H. S. Lipson und D. S. Tannhauser: Optik. Springer, Berlin, 1995.

[40] Lüders, Klaus und Robert O. Pohl: Pohls Einführung in Die Physik: Mechanik, Akustik, Wärme. Springer, Berlin, Heidelberg, New York, 2004. ISBN: 3-540-20309-5.

[41] Lutz, Holger und Wolfgang Wendt: Taschenbuch der Regelungstechnik. Harri Deutsch, Thun, Frankfurt, 1998.

[42] Martiensssen, W. (Herausgeber). Landolt-Börnstein: Numerical Data and Functional Relationships in Science and Technology - New Series. Springer, Berlin, 2003. Gegründet von H. Landolt and R. Börnstein.

[43] Mayer-Kuckuck, T.: Atomphysik. Teubner, Stuttgart, 1998.

[44] Mayer-Kuckuck, T.: Kernphysik. Teubner, Stuttgart, 1998.

[45] Meschede, D.: Gerthsen: Physik. Springer, Berlin, 2010. ISBN: 978-3-642-12893-6.

[46] Musiol, Gerhard: Kern- und Elementarteilchenphysik. VCH, Weinheim, 1988.

[47] Niedrig, Heinz: Optik, Band 3 der Reihe Bergmann-Schaefer: Lehrbuch der Experimentalphysik. Walther de Gruyter, Berlin - New York, 1993.

[48] Paus, Hans J.: Physik in Experimenten und Beispielen. Hanser, München, 2002.

[49] Povh, Bogdan: Teilchen und Kerne: Eine Einführung in Die Physikalischen Konzepte. Springer, Berlin, 2001.

[50] Purcell, Edward M.: Elektrizität und Magnetismus, Band 2 der Reihe Berkeley-Physik-Kurs. Vieweg, Braunschweig, 1989.

[51] Raith, Wilhelm: Festkörper, Band 6 der Reihe Bergmann-Schaefer: Lehrbuch der Experimentalphysik. Walther de Gruyter, Berlin - New York, 1992.

[52] Raith, Wilhelm: Teilchen, Band 4 der Reihe Bergmann-Schaefer: Lehrbuch der Experimentalphysik. Walther de Gruyter, Berlin - New York, 1992.

[53] Raith, Wilhelm: Vielteilchen-Systeme, Band 5 der Reihe Bergmann-Schaefer: Lehrbuch der Experimentalphysik. Walther de Gruyter, Berlin - New York, 1992.

[54] Raith, Wilhelm: Elektromagnetismus, Band 2 der Reihe Bergmann-Schaefer: Lehrbuch der Experimentalphysik. Walther de Gruyter, Berlin - New York, 1999.

[55] Raith, Wilhelm: Erde und Planeten, Band 7 der Reihe Bergmann-Schaefer: Lehrbuch der Experimentalphysik. Walther de Gruyter, Berlin - New York, 2001.

[56] Raith, Wilhelm: Sterne und Weltraum, Band 2 der Reihe Bergmann-Schaefer: Lehrbuch der Experimentalphysik. Walther de Gruyter, Berlin - New York, 2002.

[57] Reif, Frederic: Statistische Physik, Band 5 der Reihe Berkeley-Physik-Kurs. Vieweg, Braunschweig, 1990.

[58] Rinne, Horst: Taschenbuch der Statistik. Harri Deutsch, Thun, Frankfurt, 2. Auflage, 1977. 
[59] Schenk, Wolfgang, Waldemar Ilberg, Manfred Krötzsch, Dieter Geschke und Friedrich Kremer: Physikalisches Praktikum. Springer Spektrum, Wiesbaden, 2014. ISBN 3-540-63109-7.

[60] Schröter, W., K. H. Lautenschläger und H. Bibrack: Taschenbuch der Chemie. Harri Deutsch, Thun, Frankfurt, 1995.

[61] Schülerduden: Schülerduden: Die Physik. Dudenverlag, Mannheim, 2001.

[62] Stierstadt, Klaus, Wilhelm T. Hering und Thomas Dorfmüller: Mechanik, Relativität, Wärme, Band 1 der Reihe Bergmann-Schaefer: Lehrbuch der Experimentalphysik. Walther de Gruyter, Berlin - New York, 1998.

[63] Stöcker, Horst: Taschenbuch der Physik. Harri Deutsch, Thun, Frankfurt, 1998.

[64] Stöcker, Horst: Taschenbuch Mathematischer Formeln und Moderner Verfahren. Harri Deutsch, Thun, Frankfurt, 1999.

[65] Tipler, Paul A. und Ralph A. Llewellyn: Moderne Physik. Oldenbourg, München, 2003.

[66] Walcher, Wilhelm: Praktikum der Physik. Teubner Studienbücher: Physik. Teubner, Stuttgart, 8. Auflage, 2004. ISBN: 3-519-23038-0.

[67] Weber, H. und G. Herziger: Laser: Grundlagen und Anwendungen. Physik-Verlag, Weinheim, 1999.

[68] Weise, Klaus und Wolfgang Wöger: Meßunsicherheit und Meßdatenauswertung. Wiley-VCH, Weinheim, 1999.

[69] Westphal, W.: Praktikum der Physik. Springer, Berlin, 1984.

[70] Westphal, W. H.: Physik: Ein Lehrbuch. Springer, Berlin, 1970.

[71] Wichmann, Eyvind H.: Quantenphysik, Band 4 der Reihe Berkeley-Physik-Kurs. Vieweg, Braunschweig, 1989. 


\section{Abbildungsverzeichnis}

12.1 Der Versuch $»$ Ultraschall $\ll . \ldots \ldots \ldots$

13.1 Der Versuch $»$ Mikrowellen $\ldots \ldots \ldots \ldots$

14.1 Der Versuch $»$ Mikroskop $«$. . . . . . . . . . . . . . . . . . . . . . . . . . 24

14.2 Schematischer Strahlengang im Mikroskop . . . . . . . . . . . . . . . . . . . . . 25

15.1 Der Versuch $»$ Messung der Lichtgeschwindigkeit $\ldots$. . . . . . . . . . . . . . . . 30

16.1 Der Versuch $»$ Prismen- und Gitterspektrometer» $\ldots$. . . . . . . . . . . . . . . . . . 34

16.2 Hauptschnitt eines Prismas. . . . . . . . . . . . . . . . . . . . . 35

16.3 Basislänge eines Prismas. . . . . . . . . . . . . . . . . . . . 36

16.4 Interferenzmuster eines Gitters . . . . . . . . . . . . . . . . . . . . . . 37

17.1 Der Versuch $»$ Fresnelsche Formeln und Polarisation» $\ldots$. . . . . . . . . . . . . . . . 41

17.2 Schema zu Reflexion und Polarisation . . . . . . . . . . . . . . . . . . 42

17.3 Reflexionsvermögen in Abhängigkeit vom Einfallswinkel für $n=1,63$. . . . . . . . 44

17.4 Schematischer Aufbau zur Polarisation durch Reflexion (Polarimeter). . . . . . . . 45

18.1 Der Versuch $»$ Beugung und Interferenz von Laserstrahlung $«$. . . . . . . . . . . . . 48

18.2 Absorption, spontane und induzierte Emission. . . . . . . . . . . . . . . . . . 49

18.3 Beugung an zwei punktförmigen Öffnungen. . . . . . . . . . . . . . . . 54

18.4 Beugung am Spalt $\ldots \ldots \ldots \ldots \ldots \ldots \ldots$

18.5 Beugung am Steg. . . . . . . . . . . . . . . . . . . . . 55

18.6 Beugung an der Kreisblende. . . . . . . . . . . . . . . . . . . . 56

18.7 Beugung am Gitter. . . . . . . . . . . . . . . . . . . . 58

18.8 Intensitätsverteilung am Gitter. . . . . . . . . . . . . . . . . . . . . . . . . 59

18.9 Strahlengang für den Versuch $»$ Beugung mit Laserstrahlung $d \ldots$. . . . . . . . . . 59

19.1 Der Versuch $»$ Spezifische Elektronenladung ${ }_{4} . \ldots$. . . . . . . . . . . . . . . . . 64

20.1 Der »Franck-Hertz-Versuch $\ll$ mit Neon. . . . . . . . . . . . . . . . . . 68

20.2 Schema der Franck-Hertz-Röhre ． . . . . . . . . . . . . . . . . . . . . . . . 69

21.1 Der Versuch $»$ Balmerserie von Wasserstoff . . . . . . . . . . . . . . . . 72

22.1 Der Versuch $»$ Röntgenstrahlung $\| . \ldots$. . . . . . . . . . . . . . . . . . . . . . . 75

22.2 Schematischer Aufbau eines Röntgenspektrometers . . . . . . . . . . . . 76

22.3 Prinzip eines Geiger-Müller-Zählers _ . . . . . . . . . . . . . . . . . . . 77

22.4 Totaler Wirkungsquerschnitt von Blei. . . . . . . . . . . . . . . . . . . . . . . . . 79 
23.1 Der Versuch $»$ Zeeman-Effekt $\ll$. . . . . . . . . . . . . . . . . . . . . . . . . . 84

24.1 Der Versuch $»$ Elektronenspinresonanz $\ldots \ldots \ldots \ldots$. . . . . . . . . . . . . 91

24.2 Skizze der Spulenanordnung des Versuchs »Elektronenspinresonanz«. . . . . . . 93

$25.1 \quad$ Der Versuch $»$ Radioaktivität $«$. . . . . . . . . . . . . . . . . . . . 96

25.2 Potenzialtopf für Protonen und Neutronen . . . . . . . . . . . . . . . . . . 97 


\section{Tabellenverzeichnis}

A.1 Praktikumsbücher . . . . . . . . . . . . . . . . . . . 2

A.2 Allgemeine Physikbüchen . . . . . . . . . . . . . . . . 3

A.3 Handbücher und Nachschlagewerke $\ldots \ldots \ldots \ldots \ldots$

A.4 Fundamentalkonstanten . . . . . . . . . . . . . . . . . . . . . . 4

$12.1 \quad$ Elastische Koeffizienten . . . . . . . . . . . . . . . . . . . . . . . . . . 19

16.1 Emissionslinien von Quecksilber und Cadmium. . . . . . . . . . . . . . . . . . . . 39

$18.1 \quad$ Extrema der Besselfunktion $J_{1}(\varepsilon) . \ldots \ldots \ldots \ldots$. . . . . . . . . . . . 57

18.2 Größenangaben für die Beugungsobjekte . . . . . . . . . . . . . . . . 62

22.1 Parameter für die Aufnahme der charakteristischen Strahlung. . . . . . . . . . . 81

22.2 Parameter für die Messung der Grenzwellenlänge . . . . . . . . . . . . . . . . 81

22.3 Parameter der Messungen zu Absorptionskanten. . . . . . . . . . . . . . . . . 81

22.4 Energieniveaus der Anodenmaterialien. . . . . . . . . . . . . . . . . . 82

22.5 Charakteristische Röntgenlinien der Anodenmaterialien . . . . . . . . . . . . . 82

22.6 K-Absorptionskanten einiger Elemente . . . . . . . . . . . . . . . . 82

X.1 Raumverzeichnis der Praktikumsversuche . . . . . . . . . . . . . . . . . . . . . . 114 


\section{Raumverzeichnis des Praktikums}

Die Praktikumsräume des Göttinger Praktikums zu Experimentalphysik befinden sich im Neubau der Physik im Bauteil A. Sie verteilen sich auf die Räume A 1.101-A 1.117 und A 2.101-A 2.109. Die Versuche mit den zugehörigen Räumen sind in Tabelle X.1 aufgelistet. Eine Raumübersicht der Ebenen 1 und 2 des Praktikums ist hier dargestellt.
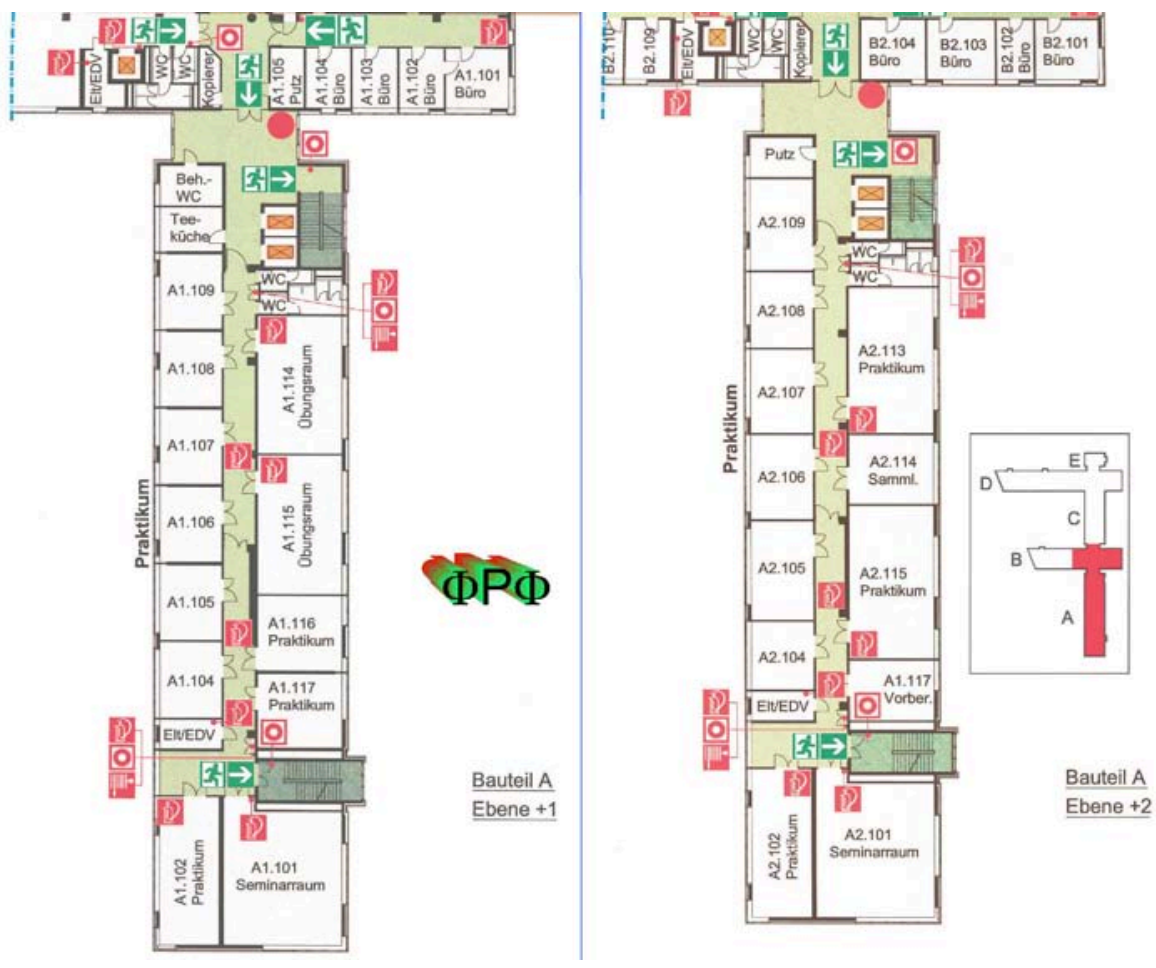
Tabelle X.1: Raumverzeichnis der Praktikumsversuche in Göttingen (M-Mechanik, W-Wärmelehre, EM-Elektrizität und Magnetismus, WO-Wellen und Optik, A-Atomphysik, S-Statistik, HHilfsmittel).

\begin{tabular}{|c|c|c|c|c|}
\hline $\mathrm{Nr}$ & & Versuch & Raum & Telefon \\
\hline 1 & M & Pohlscher Resonator & A 1.105 / A 1.106 & \\
\hline 2 & M & Trägheitsmomente & A 2.105 & \\
\hline 3 & M & Kreiselpräzession & A 1.104 & \\
\hline 4 & $\mathrm{~W}$ & Spezifische Wärme der Luft und Adiabatenexponent & A 1.102 / A 2.102 & $13253 / 13254$ \\
\hline 5 & $\mathrm{~W}$ & Dampfdruck von Wasser & A 2.102 & 13254 \\
\hline 6 & $\mathrm{~W}$ & Kapillarität und Viskosität & A 2.102 & 13254 \\
\hline 7 & EM & Coulombsches Gesetz & A 1.117 & \\
\hline 8 & EM & Wechselstromwiderstände & A 1.106 & \\
\hline 9 & EM & Magnetfeld von Spulen & A 1.109 & 13252 \\
\hline 10 & EM & Para- und Diamagnetismus & A 1.107 & \\
\hline 11 & EM & Transformator & A 1.108 & \\
\hline 12 & WO & Ultraschallwellen & A 1.116 & \\
\hline 13 & WO & Mikrowellen & A 2.106 & \\
\hline 14 & WO & Das Mikroskop & A 2.107 & \\
\hline 15 & WO & Messung der Lichtgeschwindigkeit & A 1.114 & 13251 \\
\hline 16 & WO & Das Prismen- und Gitterspektrometer & A 2.108 & \\
\hline 17 & WO & Fresnelsche Formeln und Polarisation & A 2.109 & \\
\hline 18 & WO & Beugung und Interferenz von Laserlicht & A 1.115 & \\
\hline 19 & A & Die spezifische Elektronenladung & A 1.105 & \\
\hline 20 & A & Der Franck-Hertz-Versuch & A 2.107 & \\
\hline 21 & A & Balmerserie von Wasserstoff & A 2.108 & \\
\hline 22 & A & Röntgenstrahlung & A 2.104 & \\
\hline 23 & A & Zeeman-Effekt & A 1.114 & 13251 \\
\hline 24 & A & Elektronenspinresonanz & A 1.115 & \\
\hline \multirow[t]{5}{*}{25} & $S$ & Radioaktivität (Quelle in A 1.104) & A 1.116 & \\
\hline & $\mathrm{H}$ & Multimeter/Stoppuhren-Schrank & Flur A 1 & \\
\hline & $\mathrm{H}$ & Praktikumsvorbereitung & A 2.116 & 13255 \\
\hline & & Seminarraum 1 & A 1.101 & \\
\hline & & Seminarraum 2 & A 2.101 & \\
\hline
\end{tabular}




\section{Stichwortverzeichnis}

\author{
Symbole \\ $\chi^{2}$-Methode, 95,103 \\ $\lambda, 35$ \\ $e, 64$ \\ $n, 35$
}

\section{A}

Ablenkkondensator,65

Abschirmung, 95

Absorption, 49

Absorptionskanten,75

Aktivierung, 96,104

Analysesoftware,96

Anode, 76

Anodenspannung, 66

Auflösungsvermögen, 24 34, 36

Aufräumen, 7

Auswahlregeln, 51

\section{B}

Babinetsches Theorem,55

Bahndrehimpuls, 85

Balmerserie, 73

Besetzungszahlen, 50

Beugung,48

Beugungsmuster, 36

Boltzmannverteilung, 50

Bragg-Bedingung,76

Bragg-Reflexion,75

Braunsche Röhre, 65

Brechungsindex, 3541

Bremsstrahlung, 75 [76

Brewster-Winkel,42

Bücher,2

- Allgemeine Physik, 2

- Handbücher, 3

- Nachschlagewerke, 3

- Praktikumsbücher,2

\section{C}

Charakteristische Strahlung, 75,76

\section{D}

Definitionswert,30

Dichroismus, 42

Dielektrizitätskonstante, 31

Dipol-Auswahlregel, 86

Dispersion, 34
Dopplerbreite, 52

Drehimpulsquantenzahl,85

Durchlässigkeit,43

E

Ebene Welle, 53

Effektive Basisbreite, 36

Einstein-Koeffizienten, 50

Elektron

- Ladung,64

- - spezifische,64

- Masse, 64

Elektronenspinresonanz,92

Emission

- induzierte, 50

- spontane, 50

Energiedichte, 52

Energieniveaus, 6873

Energiezustand,49

Erste Hilfe, 12

\section{F}

Fabry-Pérot-Interferometer,86

Feinstruktur,77

Fernrohr,25

Feuer,12

Fotodiode, 4448

Fotospannung, [53

Franck-Hertz-Versuch, 70

Fraunhofer Beugung,53

Fraunhofer-Formel,34,35

Freiheitsgrad, 104

Fresnelsche Formeln, 41,42

G

Gangunterschied, 56

Gefahrstoffverordnung,12

Geiger-Müller-Zählrohr,75,76, 95

Gitter, 34

Gitterfunktion,58

Gitterspektrometer,36

Glühemission, 65

Glühkathode,76

H

Halbwertszeit, 103

Helmholtz-Spule, 65 92

Hohlraumresonator,21 
Huygens, 36

I

Intensität,43

Intensität einer Welle,52

Interferenz, 48

Inversion, 51

Isotope, 95

K

Karteikarte,10

Kathodenheizung,66

Kohärenz, 34,49

Kreisblende,56

L

Landé-Faktor, 85,92

Laser, $13,48,49$

- Moden, 51

Lasermedium, 51

Lebensdauer, 50,96

Lecherleitung, 22

Lichtgeschwindigkeit, 31

Lichtquant,49

Literatur, 2

Longitudinalwellen, 17

Lorentzkraft,65

M

magnetische Quantenzahl,85

magnetisches Moment, 85

Maxwell-Gleichungen, 30, 41

Messprotokoll, 6

Mikroskop, 24] 25

Mikrowellen,21

Monochromasie, 49

Moseley-Gesetz, 83

$\mathbf{N}$

Nachholtermin, 6 10

Notfälle,12

Notrufnummern, 12

Nullrate, 103

Numerische Apertur,24, 26

O

Objektiv,25

Okular,25

$\mathbf{P}$

Permeabilität, 31

Phasenanalyse,75

Phasensprung, 55

Plancksche Strahlungsformel, 50

Plancksches Wirkungsquantum, 73 83

Poisson-Statistik, 102
Polarisation, 41

- Schwingungsebene,42

Polarisator,42

Polarisiertes Licht, 42

Poynting Vektor, 53

Praktikumsräume, 113

Prisma, 34

Prismenspektrometer, 36

Protokoll, 7

- Theorieteil, 8

$\mathbf{R}$

Radioaktivität,05

Raumplan, 113

Reflexionsverhältnis,43

Regeln,5

Resonanzbedingung, 92

Resonator,51

Röntgenröhre,75

Röntgenstrahlung,75

Röntgenverordnung RöV,13

Rydberg-Konstante, $73,78,83$

S

Sachverzeichnis, 2

Sammelpunkt, 12

Sicherheit, 12

Snelliussche Brechungsgesetz, 35,42

Snelliussches Brechungsgesetz, 18

Spaltfunktion, 58

Spektralapparat, 36

Spektralfarben, 34

Spin, 92

Stichworte, 2

Strahlenschutzverordnung StrSchV, 13

Strahlungsdichte, 50

Strahlungsübergang,70

T

Testat, 7

Theorievortrag, 6

Totzeit,77

Transversalwellen,17,41

Tubus,25

$\mathbf{U}$

Übergangswahrscheinlichkeit, 50

Ultraschallwellen, 16

Unfall, 12

UVV, 12

V

Versuchs-Testat, 7

Versuchsende, 7

Versuchsvorbereitung, [5

Vorgängerprotokolle,2 
W

Wehneltzylinder, 65

Wellenwiderstand,53

Z

Zeeman-Effekt

- anomaler, 85

- normaler, 85 
Das Handbuch 2015/2016 ist die „Anleitung“ zum Grundpraktikum für Studentinnen und Studenten der Physik an der Georg-AugustUniversität Göttingen.

Das Grundpraktikum für das Fach Physik wird begleitend zu den Vorlesungen Experimentalphysik I...IV durchgeführt und beinhaltet die Einführungsveranstaltung »Grundlagen des Experimentierens « (GdE) sowie 25 Versuche.

Dieser Band umfasst die 14 Versuche zu Experimentalphysik III und IV, welche im Regelfall im 3. und 4. Semester absolviert werden.
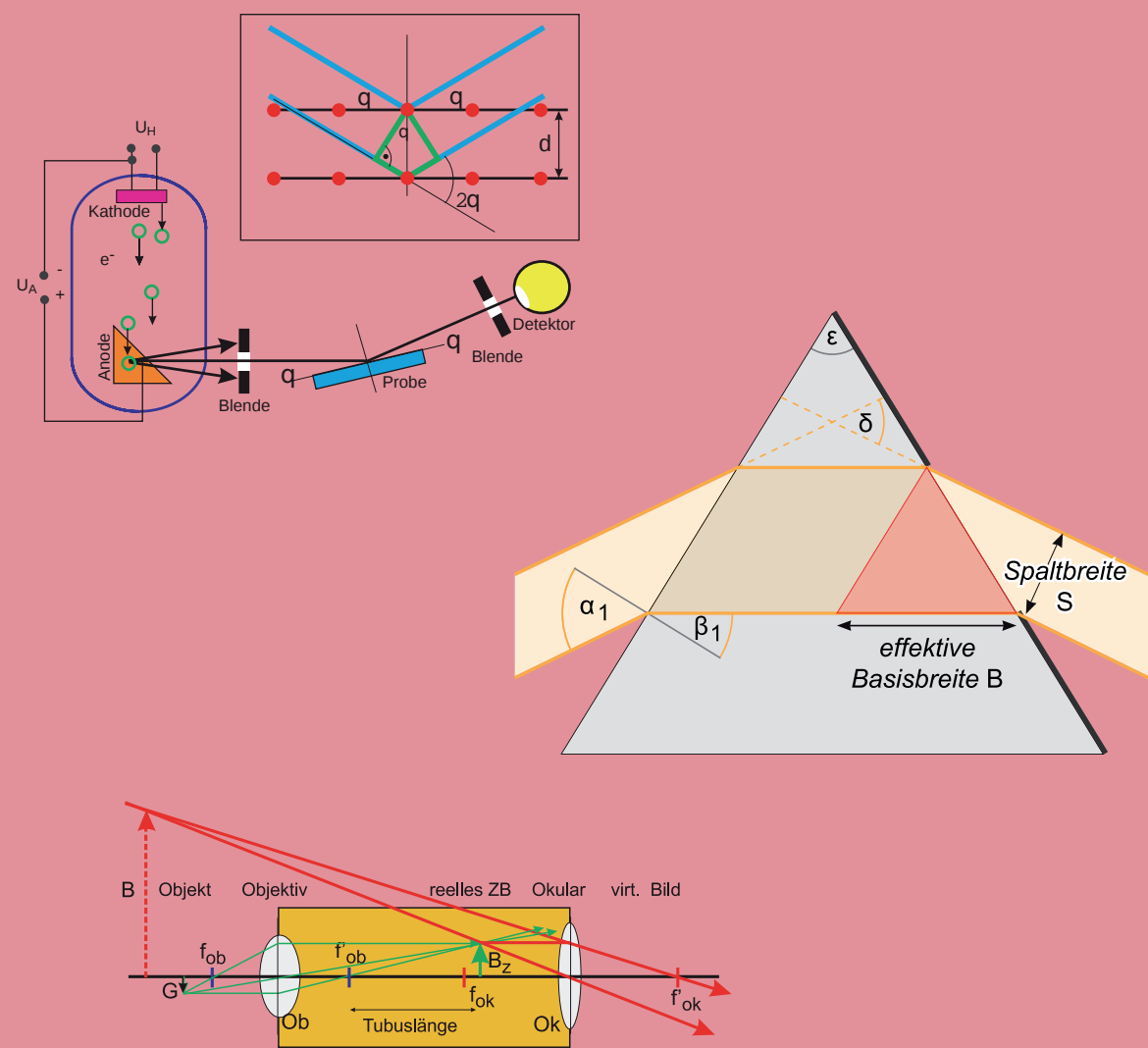